\title{
TRANSIMS Simulation Output Subsystem for 1OC-1
}

\author{
K. P. Berkbigler \\ Computer Research and Applications Group \\ Los Alamos National Laboratory \\ and \\ B. W. Bush \\ Energy and Environmental Analysis Group \\ Los Alamos National Laboratory
}

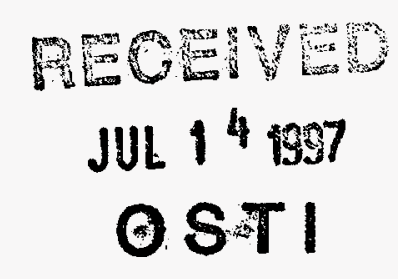

2 April 1997

\begin{abstract}
The output subsystem collects data from a running microsimulation, stores the data for future use, and manages the subsequent retrieval of the data. It forms a layer separating the other subsystems from the actual data files so that the other subsystems do not need to access the data files at the physical level or deal with the physical location and organization of the files. This subsystem also allows the user to specify what data is collected and retrieved, and to filter it by space and time. The collection occurs in a distributed manner such that the subsystem's impact on the microsimulation performance is minimized; the retrieval provides a unified view of the distributed data.
\end{abstract}

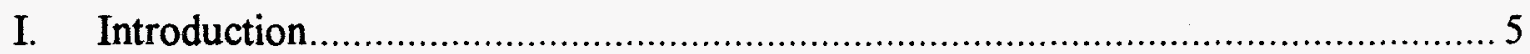

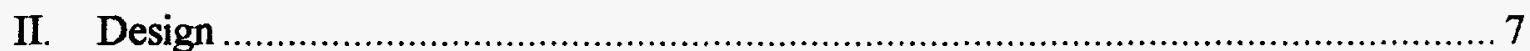

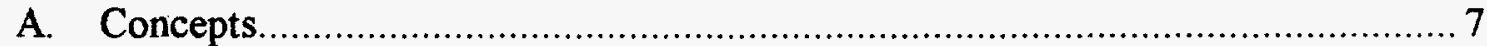

1. Types of Data Collection................................................................ 7

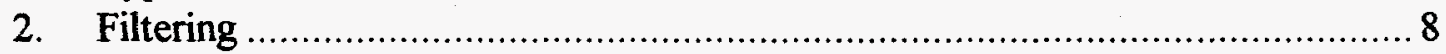

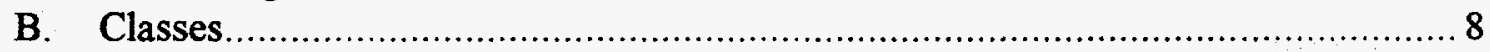

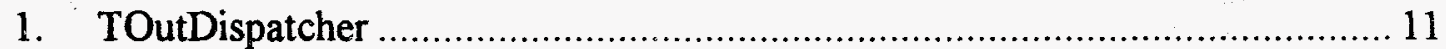

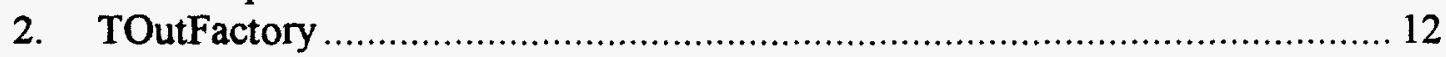

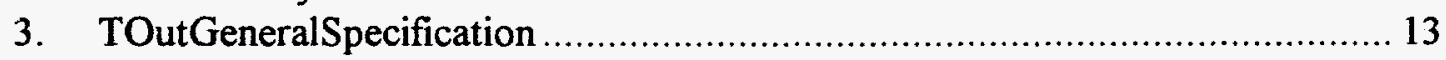

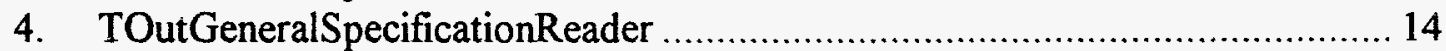

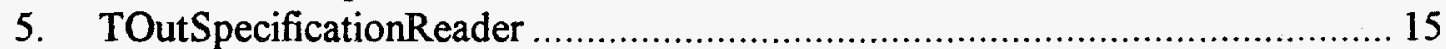

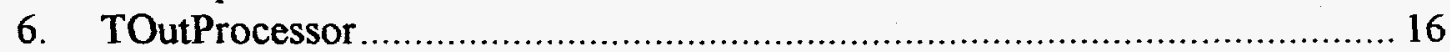

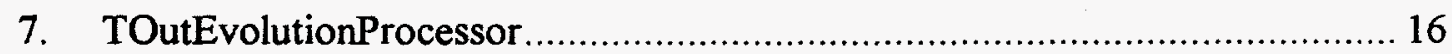

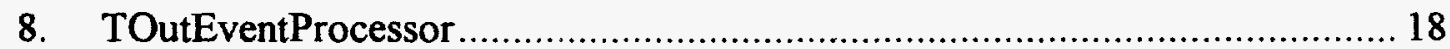

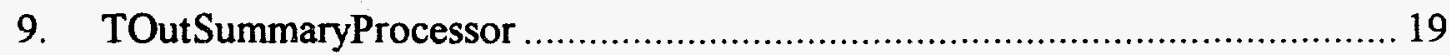

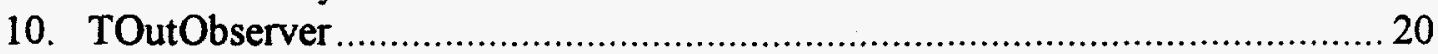

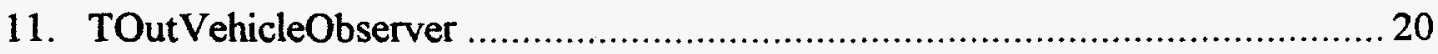

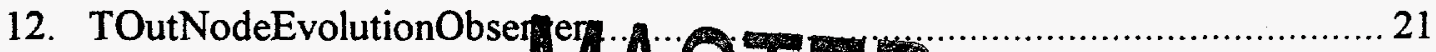




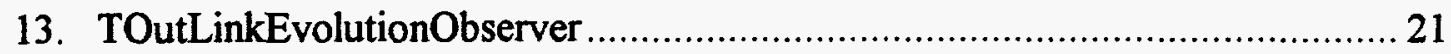

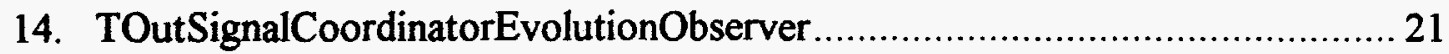

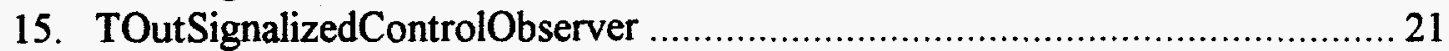

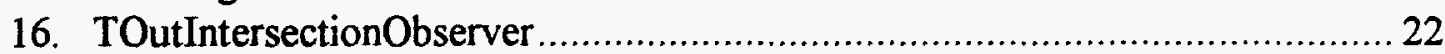

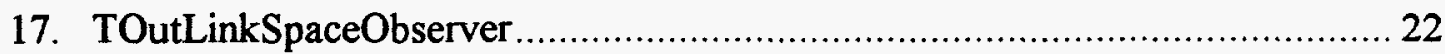

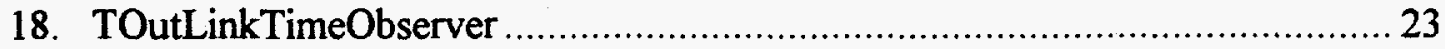

19. TOutRetriever

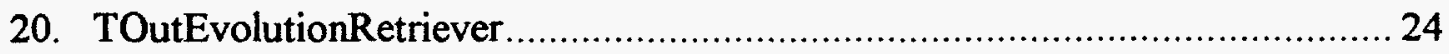

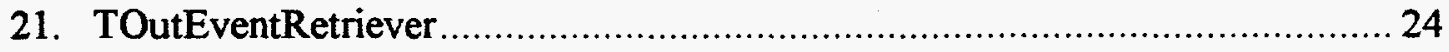

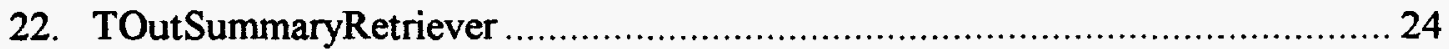

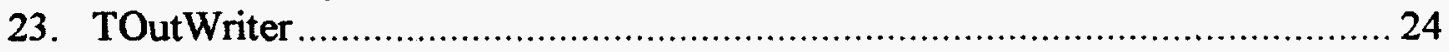

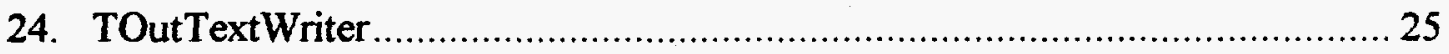

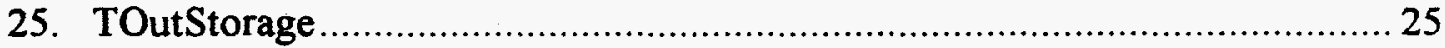

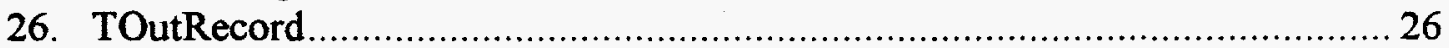

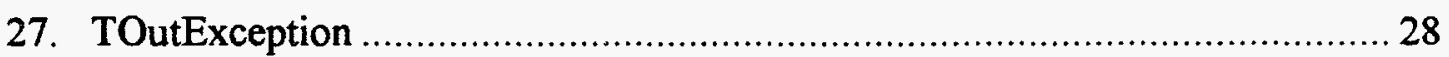

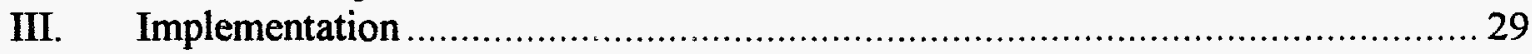

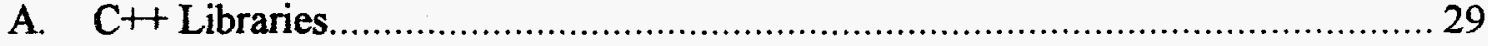

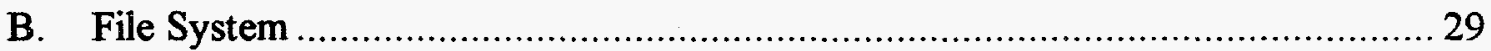

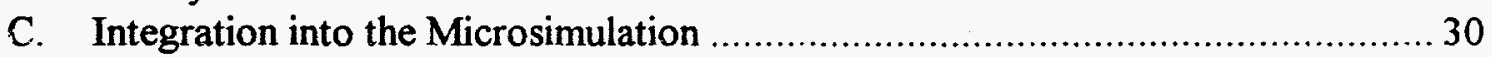

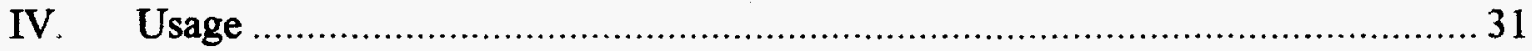

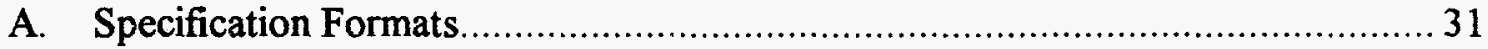

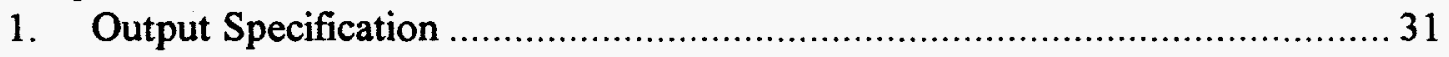

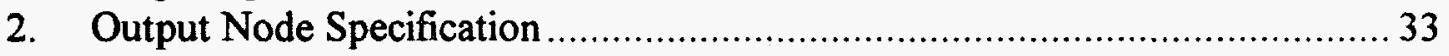

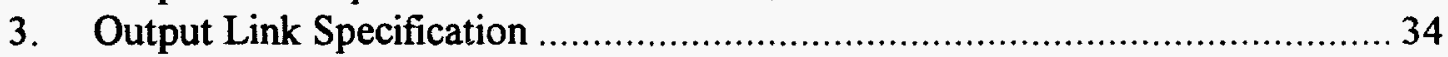

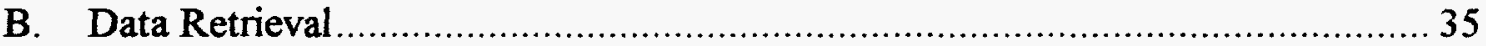

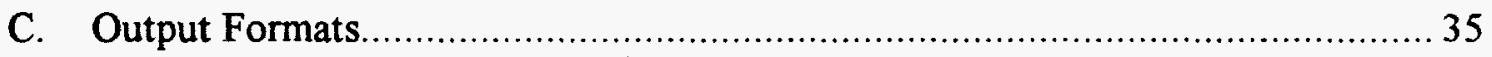

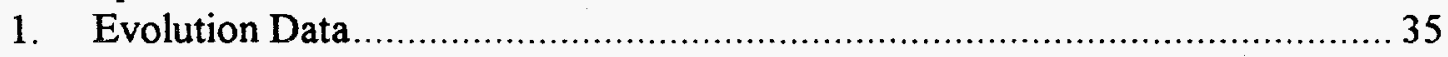

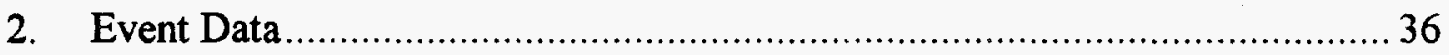

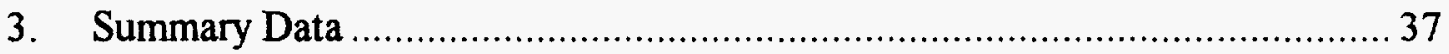

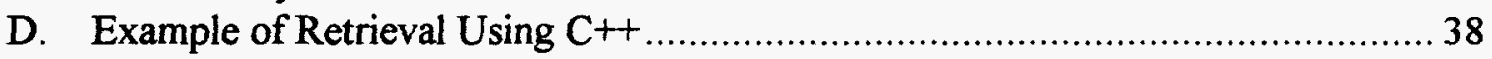

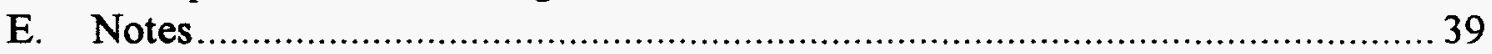

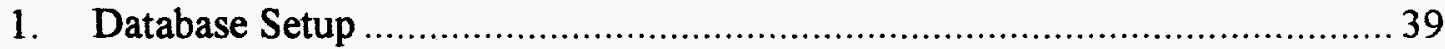

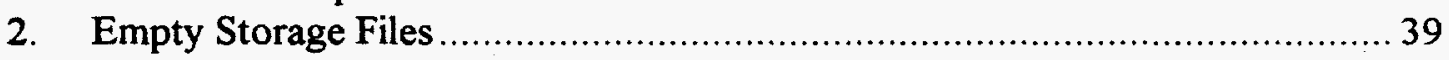

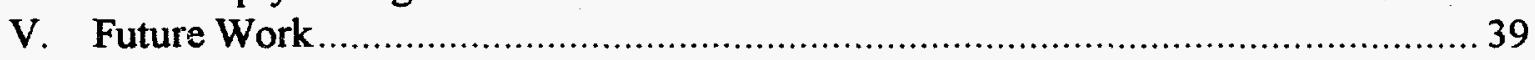

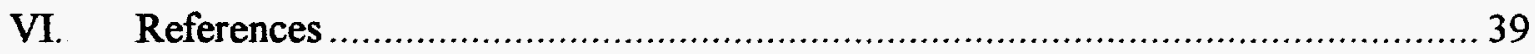

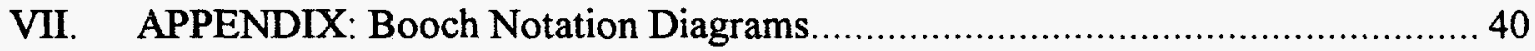

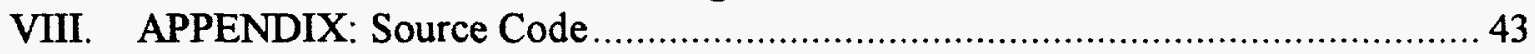

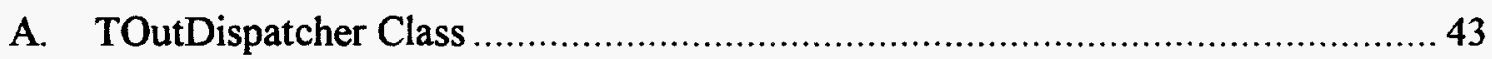

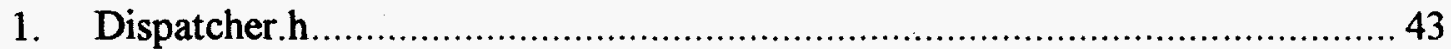

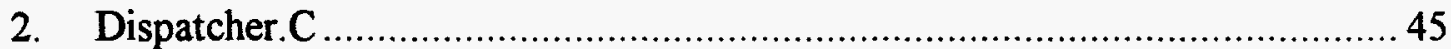

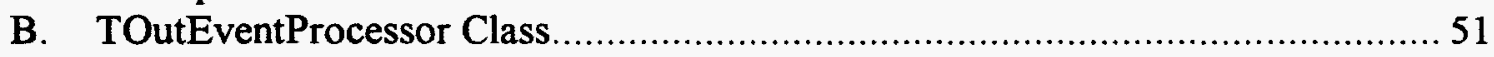

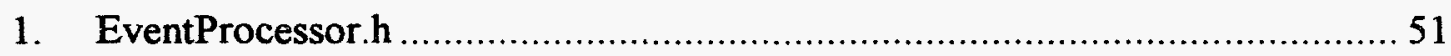

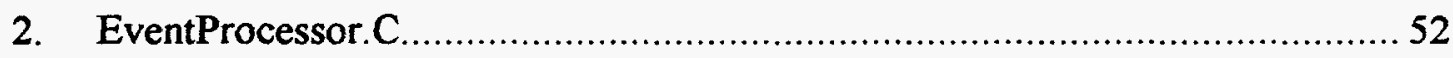

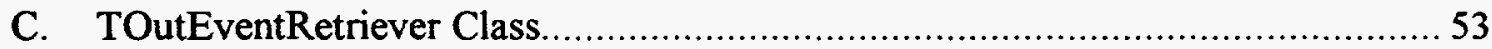

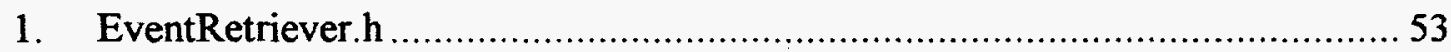




\section{DISCLAMMER}

Portions of this document may be illegible in electronic image products. Images are produced from the best available original document. 


\section{DISCLAMMER}

This report was prepared as an account of work sponsored by an agency of the United States Government. Neither the United States Government nor any agency thereof, nor any of their employees, makes any warranty, express or implied, or assumes any legal liability or responsibility for the accuracy, completeness, or usefulness of any information, apparatus, product, or process disciosed, or represents that its use would not infringe privately owned rights. Reference herein to any specific commercial product, process, or service by trade name, trademark, manufacturer, or otherwise does not necessarily constitute or imply its endorsement, recommendation, or favoring by the United States Government or any agency thereof. The views and opinions of authors expressed herein do not necessarily state or reflect those of the United States Government or any agency thereof. 



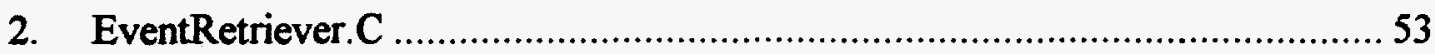

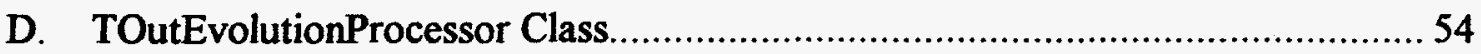

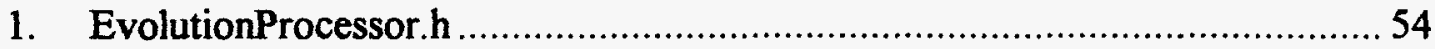

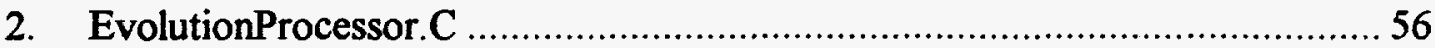

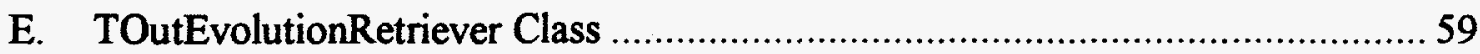

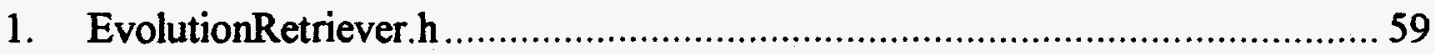

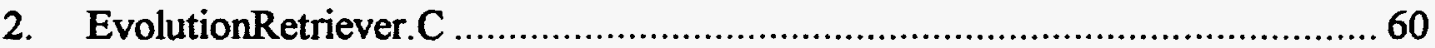

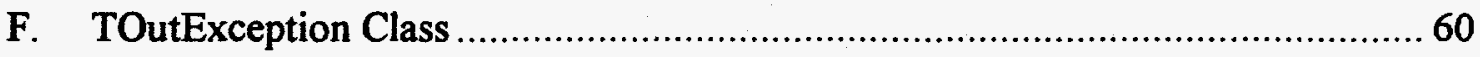

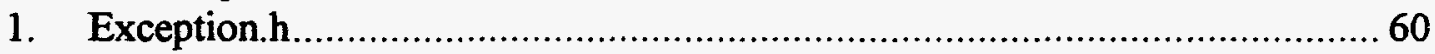

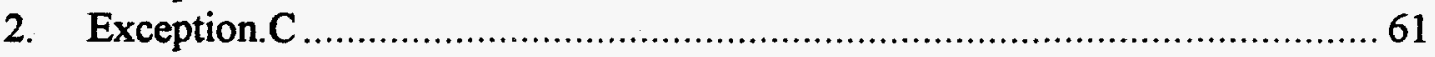

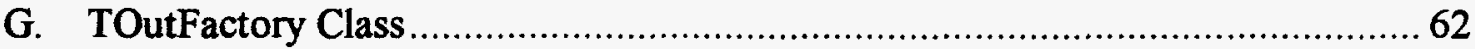

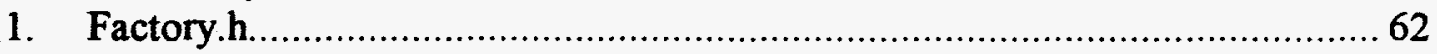

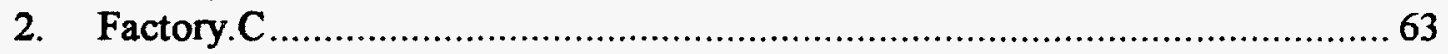

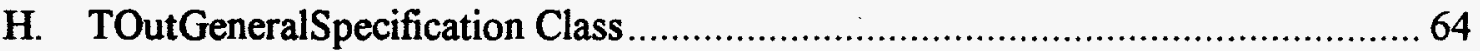

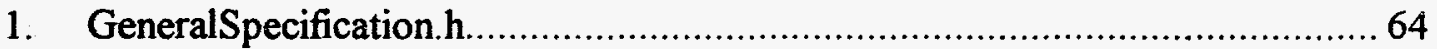

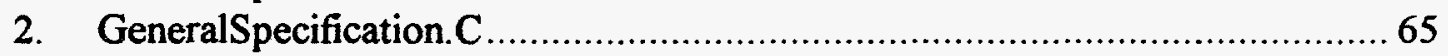

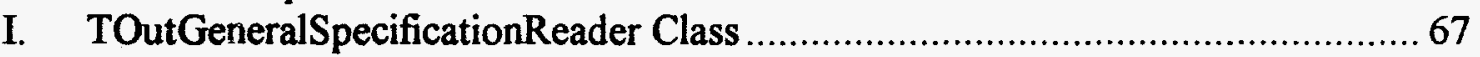

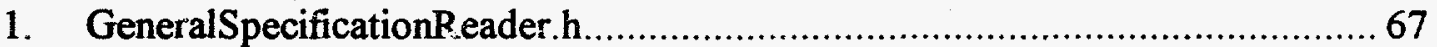

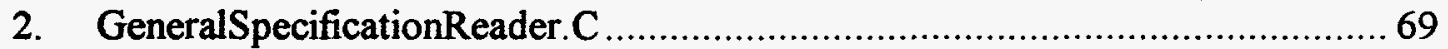

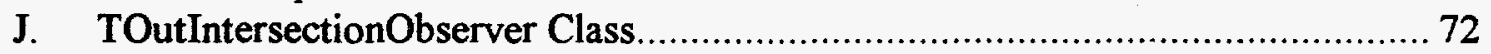

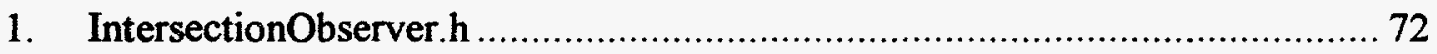

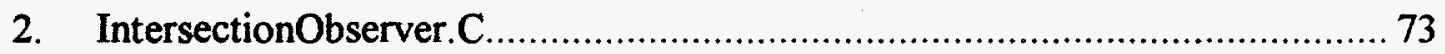

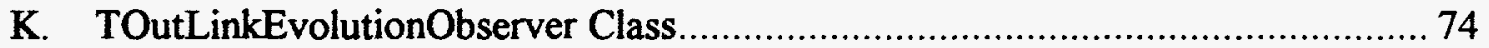

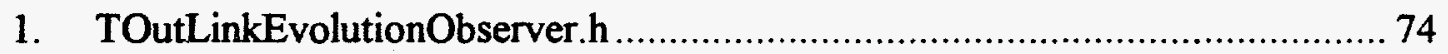

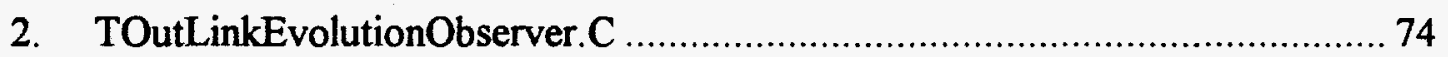

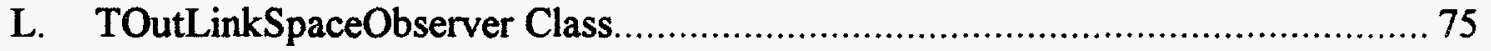

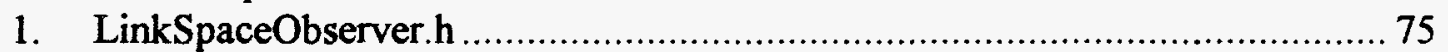

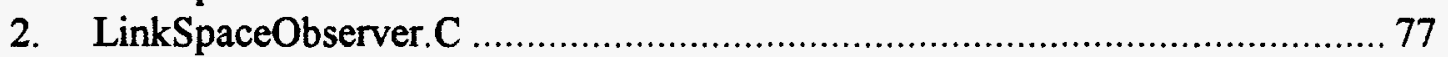

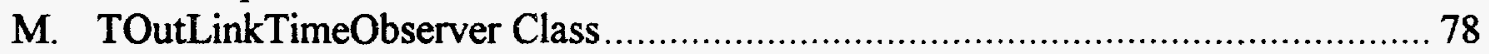

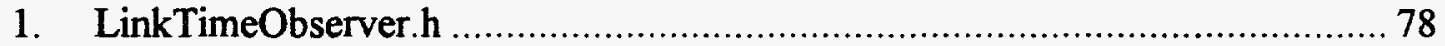

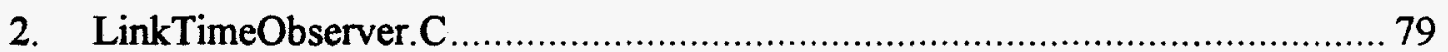

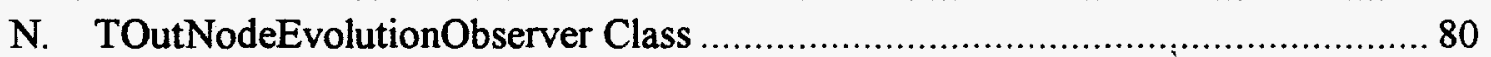

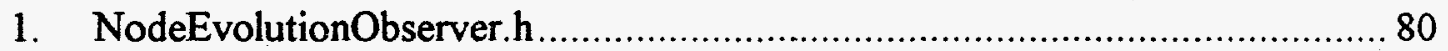

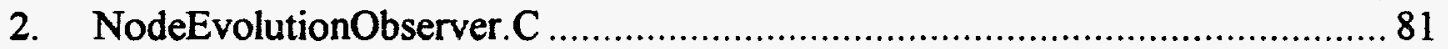

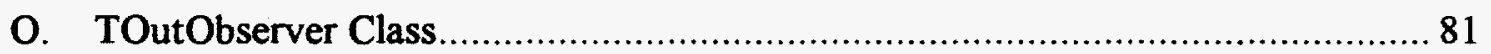

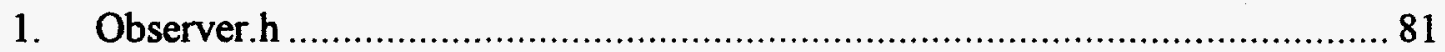

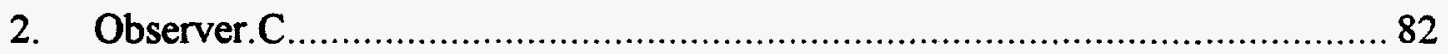

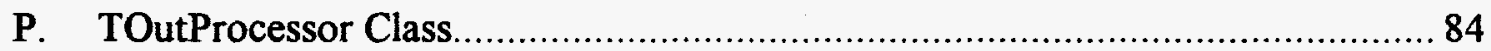

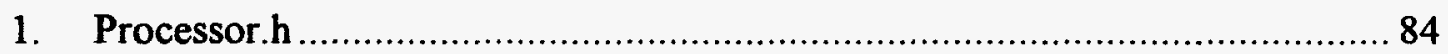

2. Processor.C …

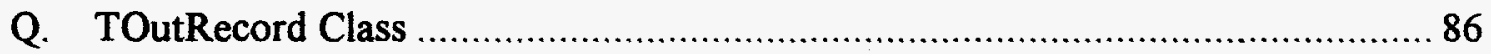

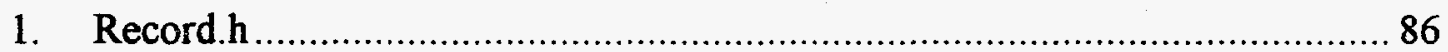

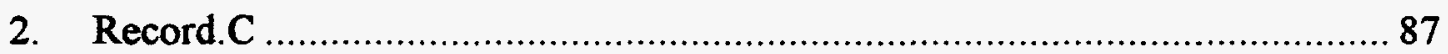

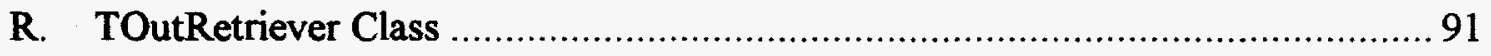

1. Retriever.h 


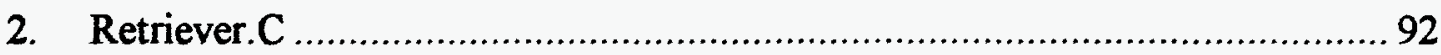

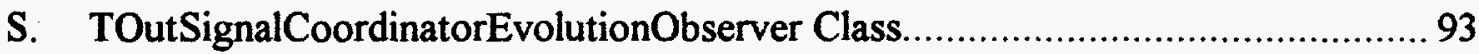

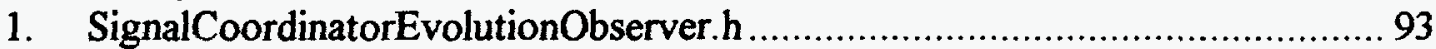

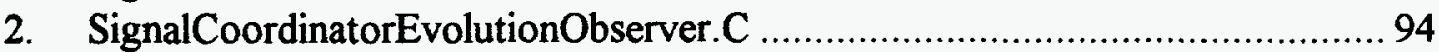

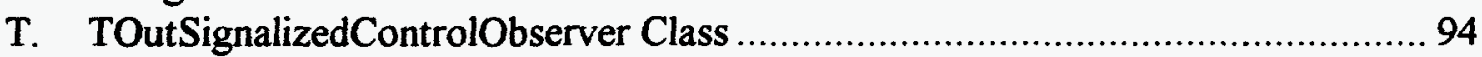

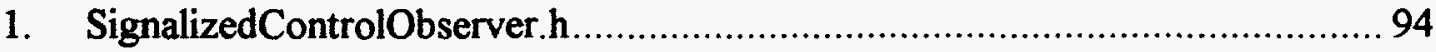

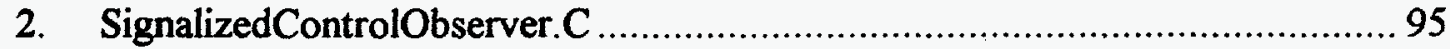

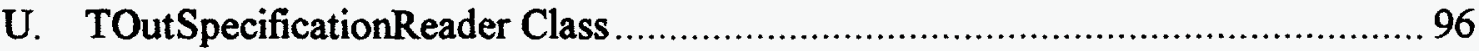

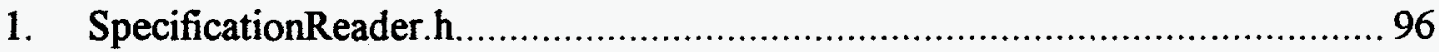

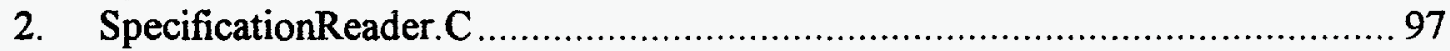

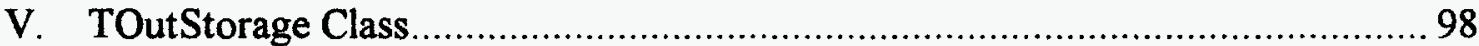

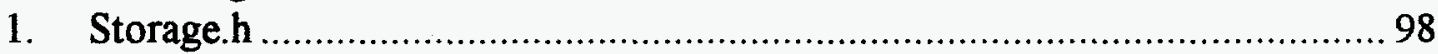

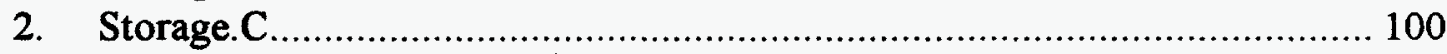

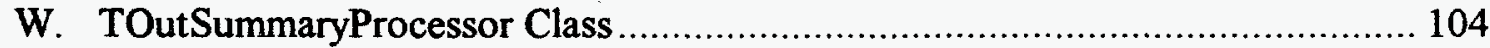

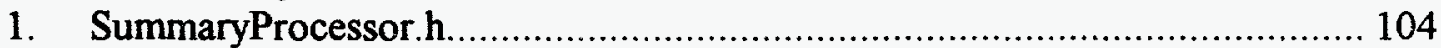

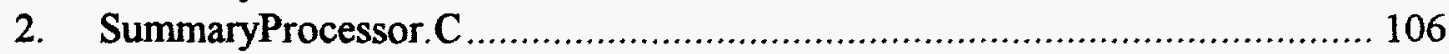

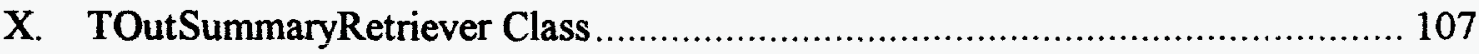

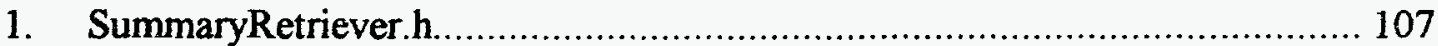

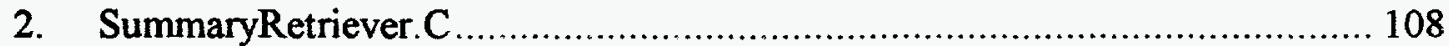

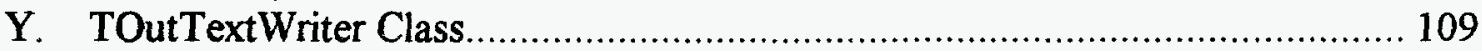

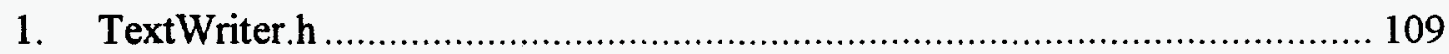

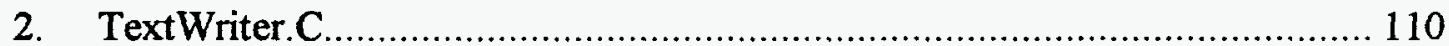

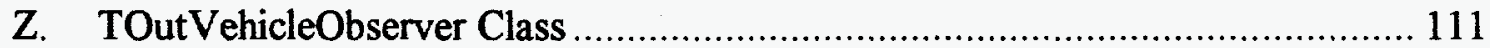

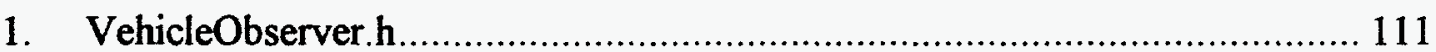

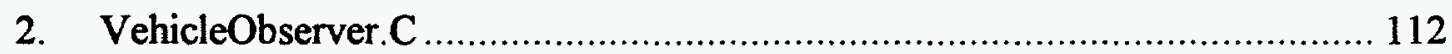

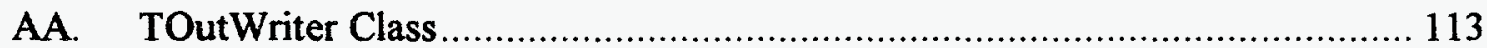

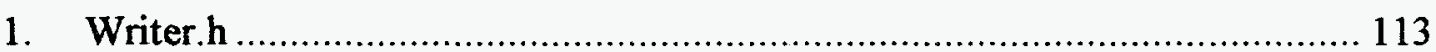

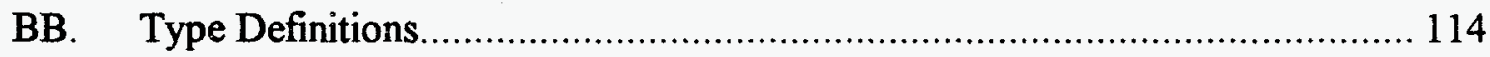

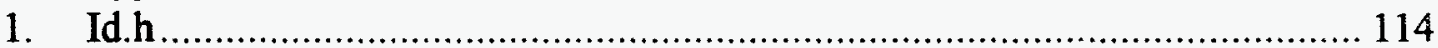

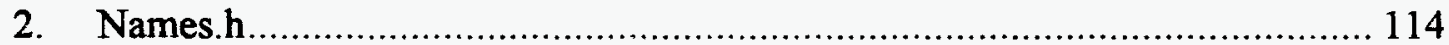

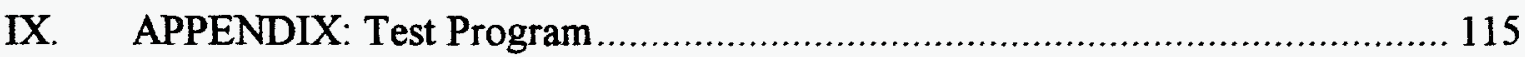

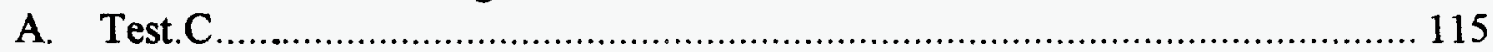

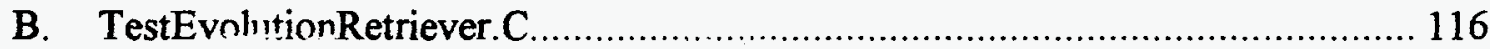

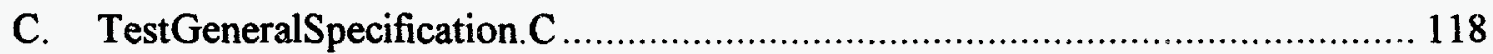

D. TestRecord.C

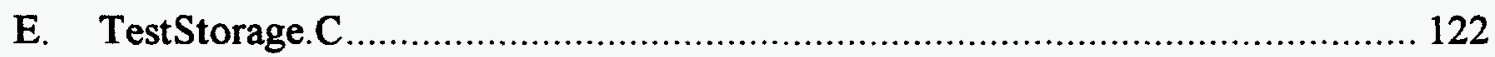

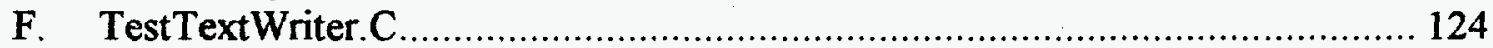

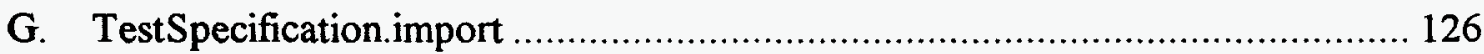

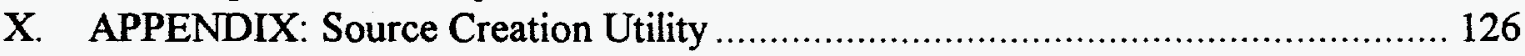

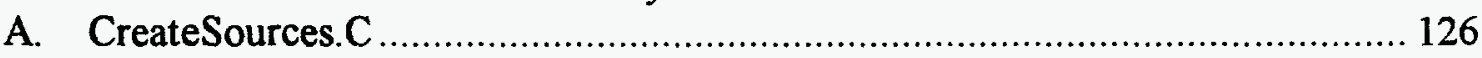

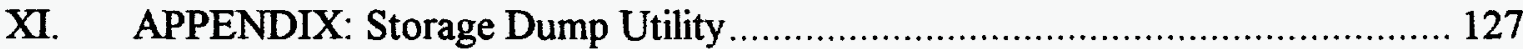

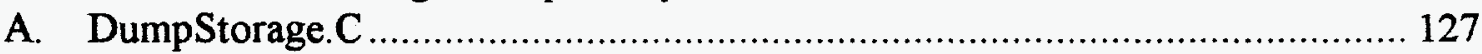




\section{Introduction}

The output subsystem collects data from a running microsimulation, stores the data for future use, and manages the subsequent retrieval of the data. It forms a layer separating the other subsystems from the actual data files so that the other subsystems do not need to access the data files at the physical level or deal with the physical location and organization of the files. Figure 1 shows the position of the simulation output subsystem within the TRANSIMS software architecture. This subsystem only depends on the database subsystem (strongly) and the network subsystem (weakly) and is not tied to the specific design used for the IOC-1 microsimulation; this opens the possibility to reuse it in other TRANSIMS traffic simulations.

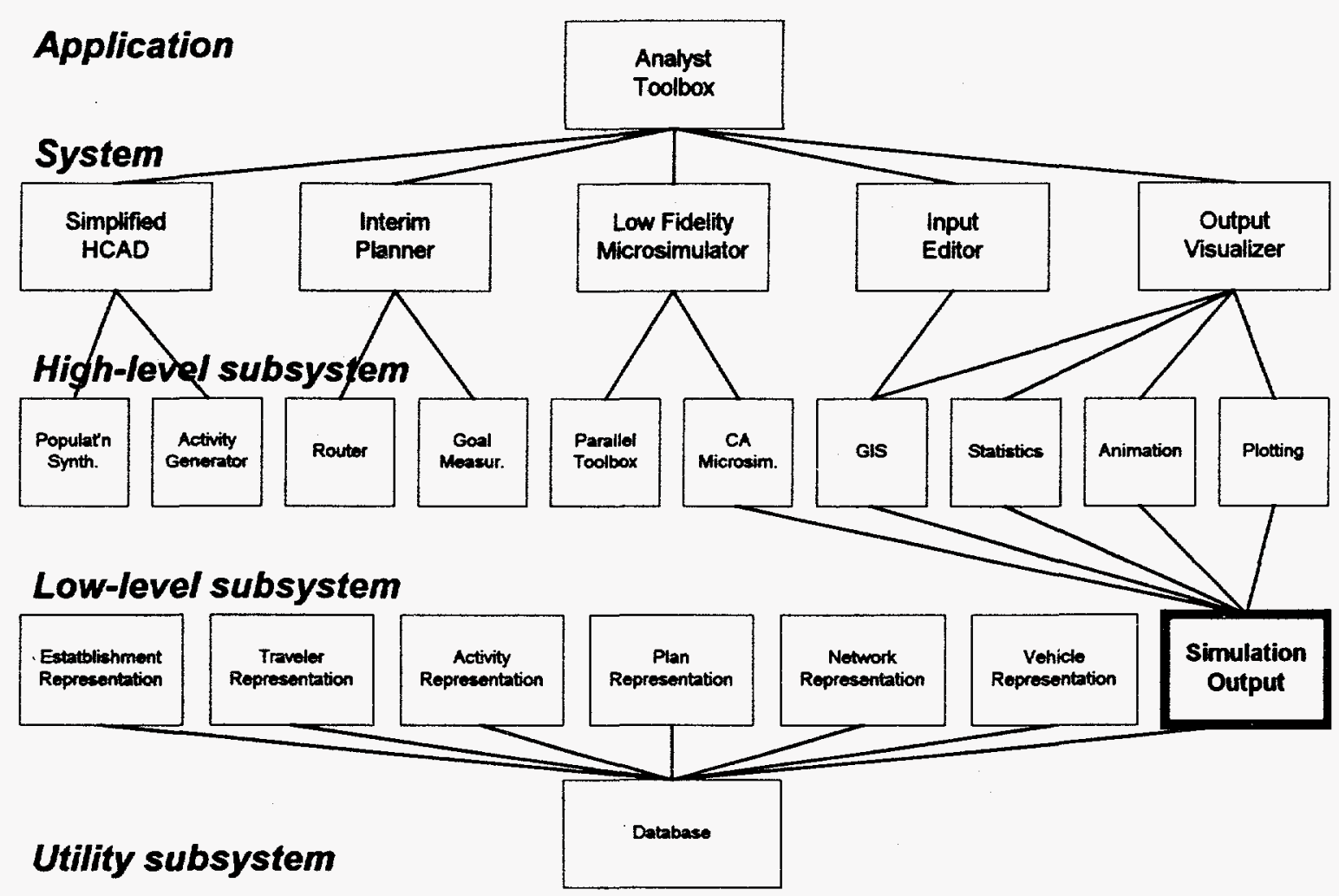

Figure 1. Location of the simulation output subsystem in the TRANSIMS software architecture.

This subsystem also allows the user to specify what data is collected and retrieved, and to filter it by space and time. Users can configure the subsystem to collect a wide variety of trajectory, event, and summary data from the simulation. Figure 2 shows an example of how data collection can be configured. The data can be accessed in binary format via a direct connection to the subsystem or in a delimited-text format for off-line postprocessing. 


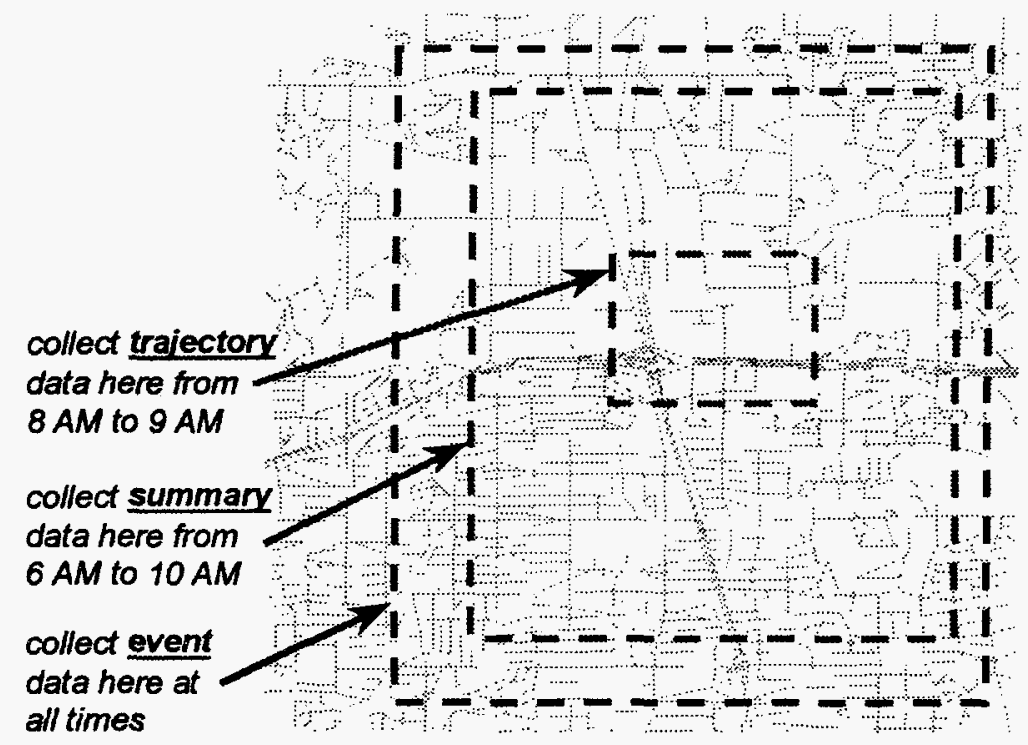

Figure 2. Example of how data collection can be filtered by space and by time.

The subsystem has been put to a wide variety of uses in the first TRANSIMS case study. Evolution data was used for animating vehicle movement, making "snapshots" of the traffic periodically, understanding the traffic behavior induced by CA rules, refining the driving logic, and deriving fundamental diagrams. Event data has helped to locate problems with network data, driver logic, and plans, and to record the entry and exit times of vehicles in and out of the study area. Summary data provided a means to animate vehicle densities, identify congestion and deadlocks, and replan trips using observed link travel times.

The collection occurs in a distributed manner such that the subsystem's impact on the microsimulation performance is minimized: Although the simulation output subsystem runs on multiple computational nodes (CPNs) during data collection, it does not require any communication between the CPNs. This leaves the full communication bandwidth available for use by the simulation proper. The retrieval, on the other hand, provides a unified view of the distributed data by coordinating the retrieval of data from remote file systems. Figure 3 illustrates the dual uses of the subsystem. 


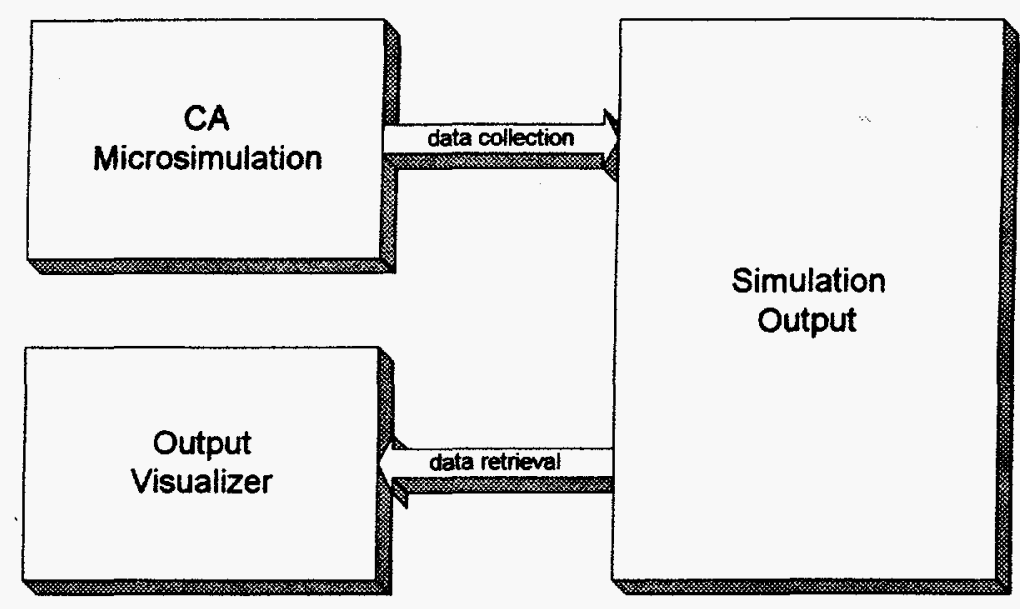

Figure 3. Dual uses of the simulation output subsystem.

The body of this document outlines the design, implementation, and usage of the subsystem. The appendices contain the complete $\mathrm{C}+i$ - source code for the subsystem.

\section{Design}

\section{A. Concepts}

\section{Types of Data Collection}

The output subsystem currently can collect three types of data: evolution (trajectory) data, event data, and summary data. Any number of each of these may be collected simultaneously in a simulation.

Evolution data provides the most detailed information about how the state of the microsimulation evolves in time. The vehicle data for links consists of the location, velocity, and status of each vehicle; this provides a complete "trajectory" for each vehicle in the simulation. The vehicle data for intersections consists of the location of the vehicle within the intersection buffer. The traffic control data simply reports the current phase and allowed movements at the traffic control. Evolution data may be collected for each time step; the data is not summarized (i.e., totaled or averaged) in any way.

Event data supplies information on exceptional conditions of vehicle status. Examples are when a vehicle becomes lost (unable to follow its plan), when the plan for a vehicle is invalid, and when the vehicle enters or exits the study area. Event data is only collected when an event occurs.

Summary data reports aggregate data about the simulation. The link travel time data consists of counts of vehicles exiting links and means and variances of the vehicle traversal times for those links. Link density data provides counts and mean velocities of vehicles in variably-sized "boxes" that partition links. Summary data is sampled and reported periodically throughout the simulation. 


\section{Filtering}

The simulation output subsystem has the capability to collect data on any subset of nodes and links in the road network (Figure 2). It is also possible to set the starting and ending times within which data collection occurs (also Figure 2). A user can also specify the frequency of reporting for evolution and summary data and the sampling frequency (i.e., the frequency at which the data is observed) for summary data. The space and time filtering can be different for each type of data.

\section{B. Classes}

The simulation output subsystem has classes containing domain knowledge and classes forming a domain-independent data management layer (Figure 4). Figure 5, Figure 6, and Figure 7, show the relationships between classes used for evolution, event, and summary data collection, respectively; Figure 8 shows the relationships between classes used for data retrieval.

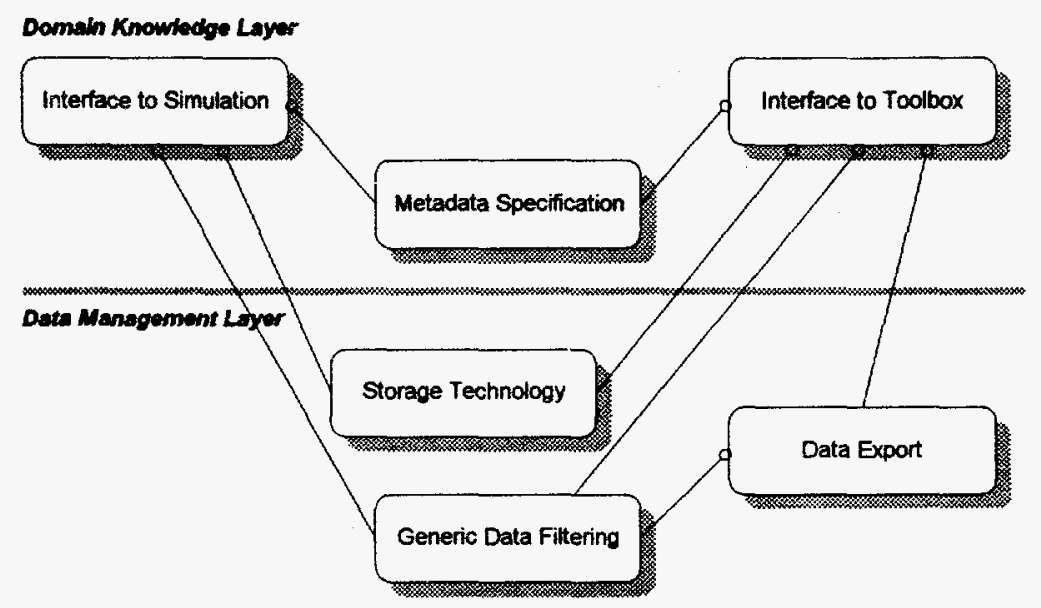

Figure 4. Categories of classes in the TRANSIMS simulation output subsystem. 


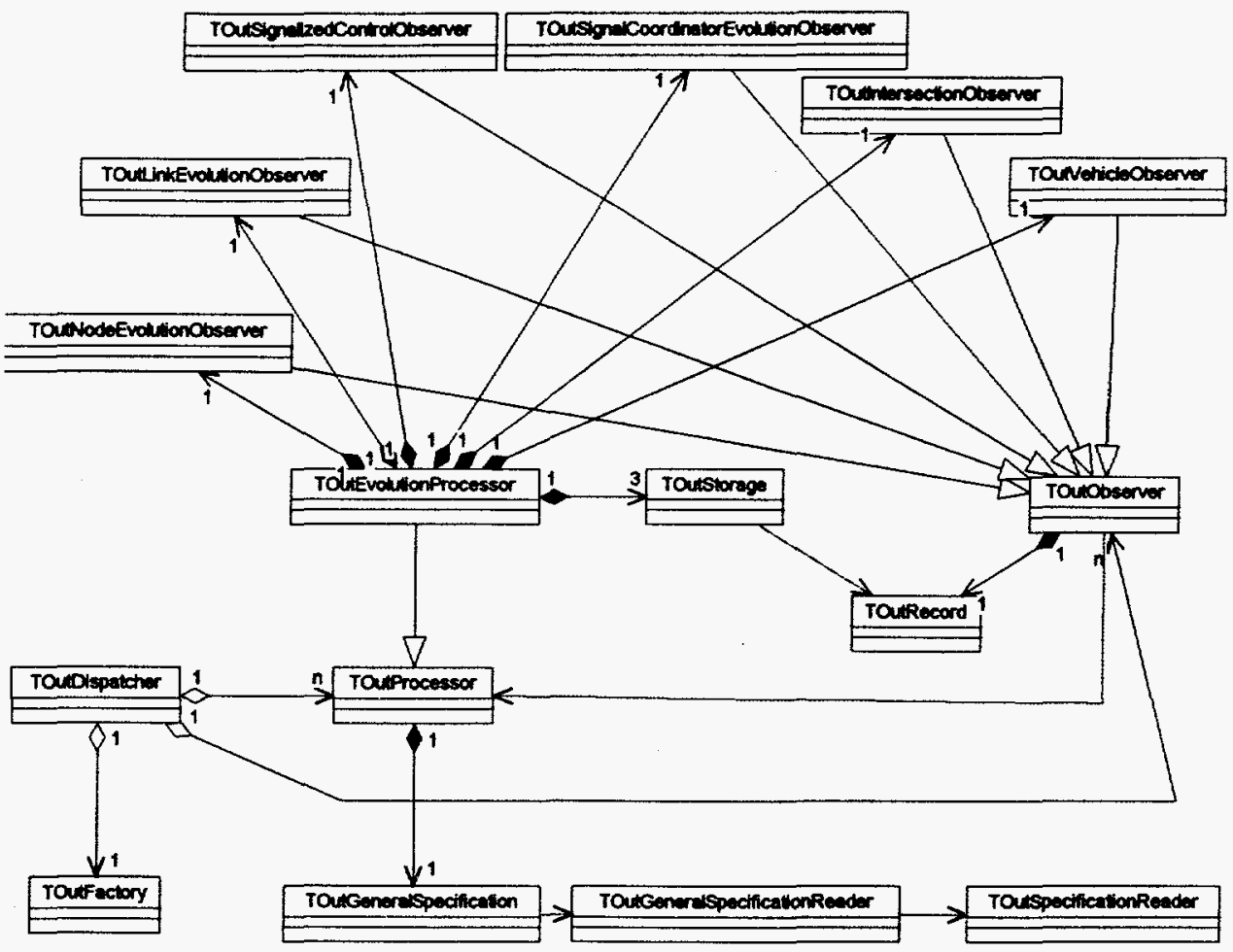

Figure 5. Class diagram for the TRANSIMS simulation output subsystem classes involved in evolution data collection (unified notation).

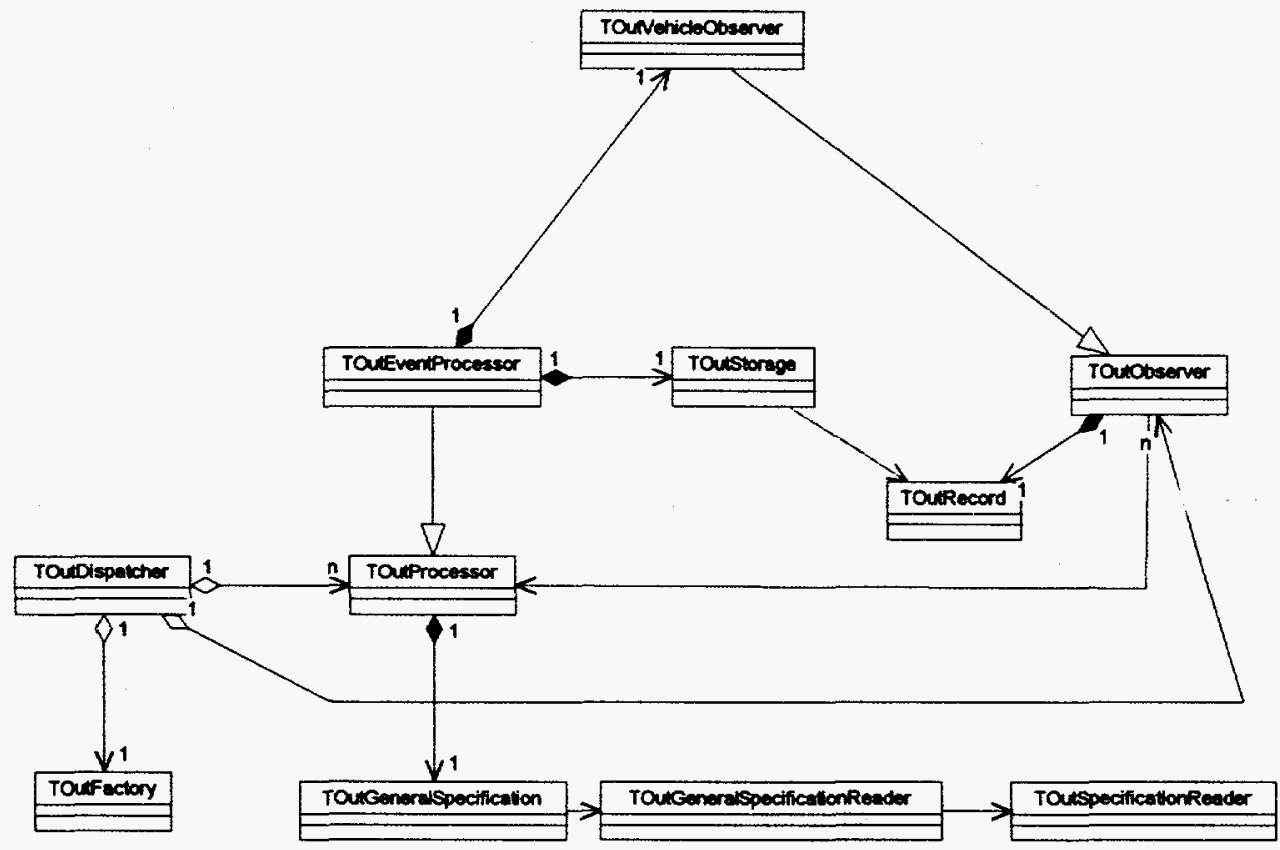

Figure 6. Class diagram for the TRANSIMS simulation output subsystem classes involved in event data collection (unified notation). 


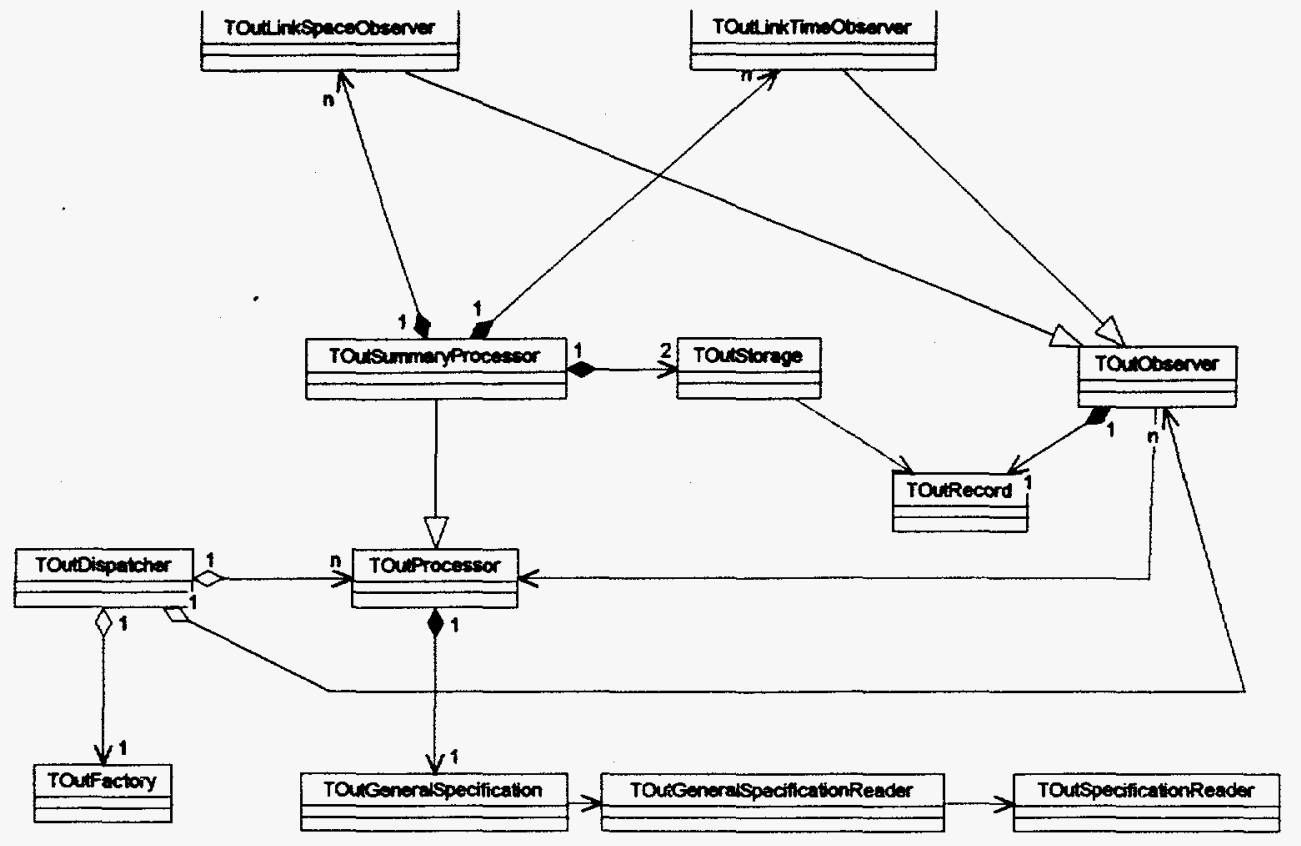

Figure 7. Class diagram for the TRANSIMS simulation output subsystem classes involved in summary data collection (unified notation).

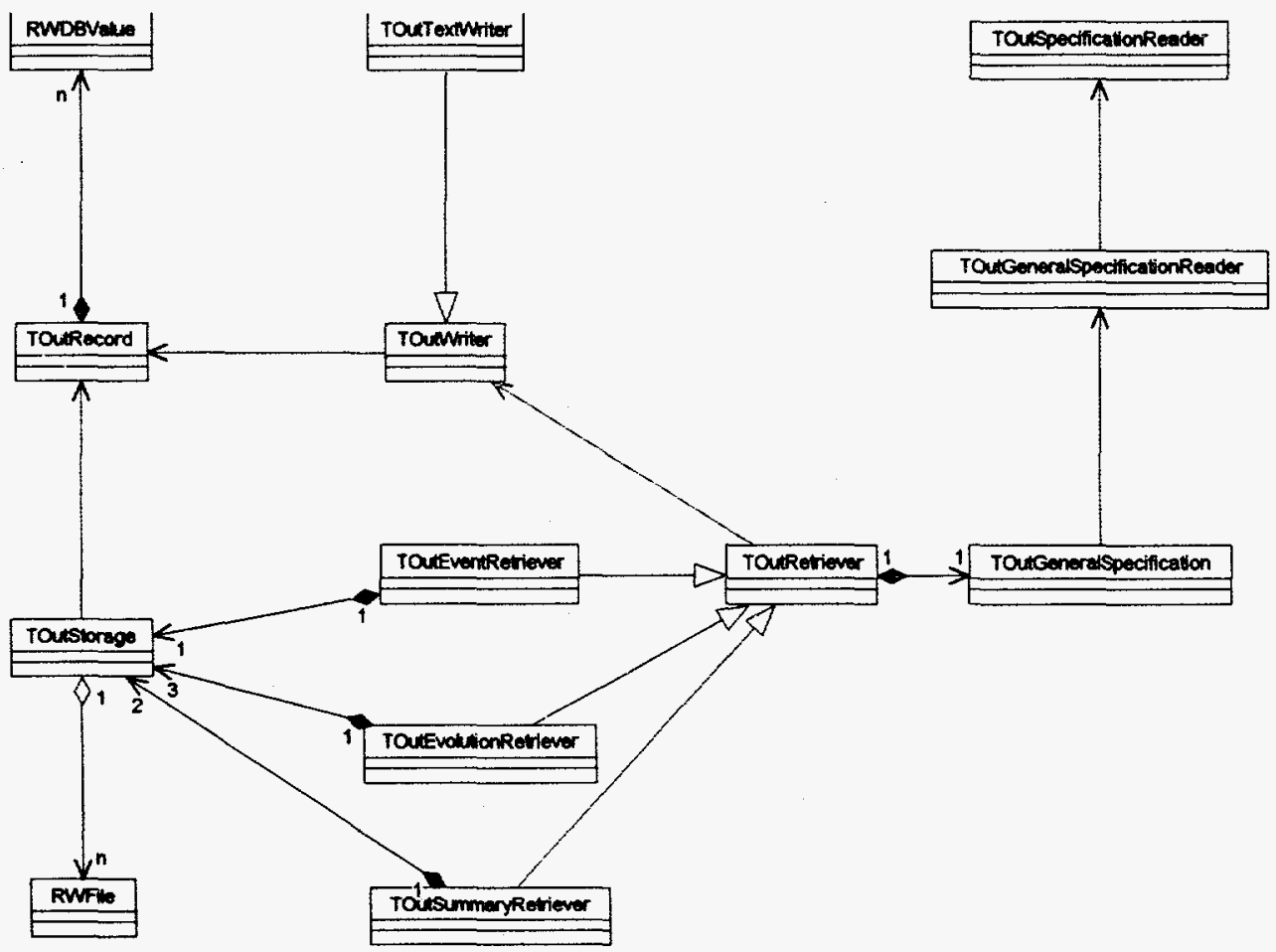

Figure 8. Class diagram for the TRANSIMS simulation output subsystem classes involved in data retrieval (unified notation). 


\section{TOutDispatcher}

An output dispatcher coordinates the construction of simulation output objects and supervises the transfer of data. Each dispatcher has processors and observers; it also remembers the factory it uses to construct objects.

enum EObserverType \{kNodeEvolutionobserver, kLinkEvolutionobserver, kVehicleobserver, kIntersectionobserver, kSignalCoordinatorEvolutionobserver, kSignalizedControlobserver, kLinkspaceobserver, kLinkTimeobserver\}

Observer types.

TOutDispatcher (TOutSpecificationReader\& reader, TOutFactory\& factory)

Construct an output dispatcher.

bool operator $==$ (const ToutDispatcher\& dispatcher) const bool operator!= (const ToutDispatcher\& dispatcher) const

Return whether two output dispatchers are the same.

void Recordoutput (REAL time)

Begin output recording for this time step.

ProcessorMap\& GetProcessors()

const ProcessorMap\& GetProcessors() const

Return the processors.

ObserverMap\& Getobservers()

const ObserverMap\& Getobservers() const

Return the observers.

EvolutionProcessorSet\& GetEvolutionProcessors()

const EvolutionProcessorSet\& GetEvolutionProcessors() const

Return the evolution processors.

EventProcessorSet\& GetEventProcessors()

const EventProcessorset\& GetEventProcessors() const

Return the event processors.

SummaryProcessorset\& GetsummaryProcessors()

const SummaryProcessorset\& GetSummaryProcessors() const

Return the summary processors.

void SetNodeObservers (TOutObserver: : ObserverMap\& observers)

Define the node observers.

void SetLinkobservers (TOutobserver: : ObserverMap\& observers)

Define the link observers. 
void SetVehicleobservers (TOutobserver: : observerMap\& observers)

Define the vehicle observers.

void SetIntersectionobservers (Toutobserver: : ObserverMap\& observers)

Define the intersection observers.

void SetSignalCoordinatorobservers (TOutobserver: : ObserverMap\& observers)

Define the signal coordinator observers.

void SetSignalizedControlobservers (TOutobserver: : ObserverMap\& observers)

Define the signalized control observers.

void SetLinkSpaceobservers (TOutobserver: : ObserverMap\& observers)

Define the link space observers.

void SetLinkTimeobservers (TOutobserver: :ObserverMap\& observers)

Define the link time observers.

void ClearLinkSpaceobservers (TOutobserver: :ObserverMap\& observers)

Undefine the link space observers.

void ClearLinkTimeobservers (TOutobserver: : ObserverMap\& observers)

Undefine the link time observers.

\section{TOutFactory}

An output factory allocates and constructs new simulation output objects.

ToutFactory()

Construct a factory.

virtual ToutEvolutionProcessor*

NewEvolutionProcessor (OutProcessorId id, const ToutGeneralspecification\& specification)

Return a new evolution processor from the specification.

virtual TOutEventProcessor* NewEventProcessor(OutProcessorId id, const ToutGeneralspecification\& specification)

Return a new event processor from the specification.

virtual ToutSummaryProcessor* NewSummaryProcessor (OutProcessorId id, const ToutGeneralspecification\& specification)

Return a new summary processor from the specification. 
virtual ToutLinkEvolutionobserver*

NewIinkEvolutionobserver (OutObserverId id)

Return a new link evolution observer.

virtual ToutVehicleobserver* NewVehicleobserver (OutobserverId id)

Return a new vehicle observer.

virtual ToutNodeEvolutionobserver*

NewNodeEvolutionobserver (OutobserverId id)

Return a new node evolution observer.

virtual ToutIntersectionobserver*

NewIntersectionobserver (OutobserverId id)

Return a new intersection observer.

virtual ToutSignalCoordinatorEvolutionobserver*

NewSignalCoordinatorEvolutionobserver (OutobserverId id)

Return a new signal coordinator evolution observer.

virtual ToutsignalizedControlobserver*

NewSignalizedControlobserver (OutobserverId id)

Return a new signalized control observer.

virtual TOutLinkspaceobserver*

NewLinkSpaceobserver (OutobserverId id)

Return a new link space observer.

virtual TOutLinkTimeobserver* NewLinkTimeobserver(OutobserverId id);

Return a new link time observer.

\section{TOutGeneralSpecification}

The general specification defines the frequency and extent of data to be collected or retrieved in both space and time. Each specification has a root, a name, a minimum time, a maximum time, a time step, a time sample, a box length, a collection region, a set of node ids, and a set of link ids.

static const REAL kMinusInfinity

static const REAL kPlusinfinity

Time constants.

TOutGeneralSpecification (TOutGeneralSpecificationReader\& reader)

Construct a general specification from a reader.

const string\& GetRoot() const

Return the root for the specification. 
const string \& GetName() const

Return the name for the specification.

bool CollectForTime (REAL time) const

Return whether data should be collected for the specified time.

bool sampleForTime(REAL time) const

Return whether data should be sampled at the specified time.

bool IsAtstartTime(REAL time) const

Return whether the specified time is the start time.

REAL GetBoxLength() const

Return the box length.

bool CollectForPoint (const TGeoPoint\& point) const

Return whether data should be collected for the specified point in space.

bool CollectForNode (NetNodeId id) const

Return whether data should be collected for the specified node.

bool CollectForLink(NetLinkId id) const

Return whether data should be collected for the specified link.

\section{TOutGeneralSpecificationReader}

A general specification reader reads specifications from the database. Each general specification reader has database table accessors for the specification and for node and link tables.

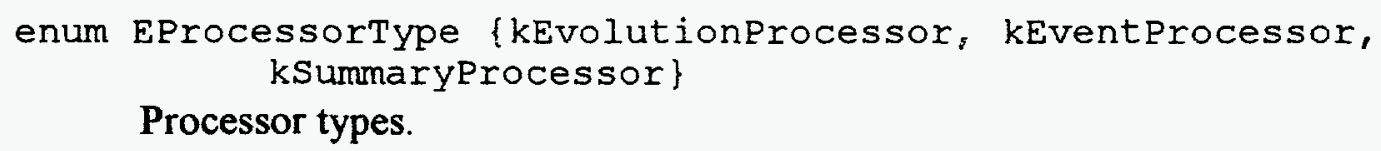

ToutGeneralspecificationReader (TOutSpecificationReader\& reader)

Construct a general specification reader.

TNetGeneralspecificationReader (const TNetGeneralspecificationReader \& reader)

Construct a copy of the given general specification reader.

TNetGeneralSpecificationReader \& operator $=($ const

TNetGeneralspecificationReader\& reader)

Make the reader a copy of the given general specification reader.

void Reset ()

Reset the iteration over the table. 
void GetNextSpecification()

Get the next specification in the table.

bool MoreSpecifications() const

Return whether there are any more specifications in the table.

string GetRoot() const

Return the root of the specification.

string GetName() const

Return the name of the specification.

REAL GetMinimumTime() const

Return the minimum time of the specification.

REAL GetMaximumTime() const

Return the maximum time of the specification.

REAL GetTimestep() const

Return the time step of the specification.

REAL GetTimeSample() const

Return the time sampling of the specification.

REAL GetBoxLength() const

Return the box length of the specification.

TGeoRectangle GetRegion() const

Return the geographic region of the specification.

NodeIdSet GetNodes() const

Return the nodes in the specification.

LinkIdSet GetLinks() const

Return the links in the specification.

EProcessorType GetProcessorType() const

Return the processor type in the specification. A TOutInvalidprocessor exception is thrown if the processor is not a valid type.

\section{TOutSpecificationReader}

A specification reader reads an output specification from the database. Each reader has a general specification table, a node specification table, and a link specification table.

ToutSpecificationReader(TDbTable generalTable, TDbTable nodeTable, TDbTable IinkTable)

Construct a reader for the specified tables. 
TDbTable\& GetGeneralTable()

Return the general specification table.

TDbTable\& GetNodeTable()

Return the node specification table.

TDbTable\& GetLinkTable()

Return the link specification table.

\section{TOutProcessor}

An output processor coordinates the processing of domain information into a domainindependent representation that is filtered and summarized before storage. A processor has an id and a general output specification; the class keeps track of next available processor id.

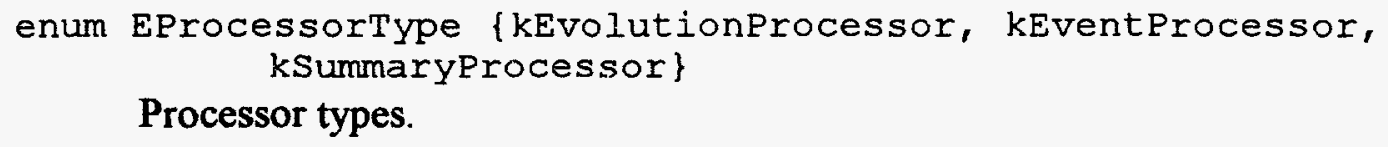

Processor types.

TOutProcessor(OutProcessorId id, const TOutGeneralspecification\& specification)

Construct a processor.

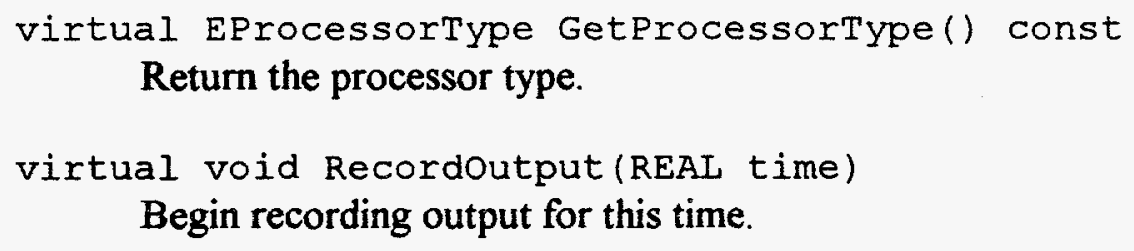

\section{TOutEvolutionProcessor}

An output evolution processor deals with evolving data such as that needed for animation, waterfall plots, etc. An evolution processor has a node observer, a link observer, a vehicle observer, an intersection observer, a signal coordinator observer, and a signalized control observer, and is connected to corresponding storage objects. 
TOutEvolutionProcessor (OutProcessorId, const ToutGeneralspecification specification)

Construct an evolution processor.

EProcessorType GetProcessorType() const

Return the processor type.

TOutEvolutionspecification\& GetEvolutionspecification()

const TOutEvolutionspecification\& GetEvolutionspecification() const

Return the evolution specification.

virtual void Recordoutput (REAL time)

Begin recording output for this time step.

virtual void RecordNode()

Finish recording output for a node.

virtual void RecordLink()

Finish recording output for a link.

virtual void RecordVehicle()

Finish recording output for a vehicle.

virtual void Recordintersection()

Finish recording output for an intersection.

virtual void RecordSignalCoordinator()

Finish recording output for a signal coordinator.

virtual void RecordSignalizedControl()

Finish recording output for a signalized control.

TOutobserver\& GetNodeobserver()

const TOutobserver \& GetNodeobserver() const

Return the node observer.

void SetNodeobserver (TOutobserver\& observer)

Define the node observer.

Toutobserver\& GetLinkobserver()

const TOutobserver\& GetLinkobserver() const

Return the link observer.

void SetLinkobserver (Toutobserver observer)

Define the link observer. 
TOutobserver\& GetVehicleobserver()

const Toutobserver\& GetVehicleobserver() const

Return the vehicle observer.

void SetVehicleobserver (TOutobserver\& observer)

Define the vehicle observer.

TOutobserver\& GetIntersectionobserver()

const Toutobserver\& GetIntersectionobserver() const

Return the intersection observer.

void SetIntersectionobserver (TOutobserver\& observer)

Define the intersection observer.

Toutobserver\& GetsignalCoordinatorobserver()

const Toutobserver\& GetSignalcoordinatorobserver() const

Return the signal coordinator observer.

void SetSignalCoordinatorobserver (TOutobserver\& observer)

Define the signal coordinator observer.

TOutObserver\& GetSignalizedControlobserver()

const TOutobserver\& GetSignalizedControlobserver() const

Return the signalized control observer.

void SetSignalizedControlobserver (TOutobserver\& observer)

Define the signalized control observer.

\section{TOutEventProcessor}

An output event processor deals with conditions occurring in the simulation such as vehicle entry, vehicle exit, and lost vehicles. An event processor has a vehicle observer and a vehicle status mask and is connected to a vehicle storage.

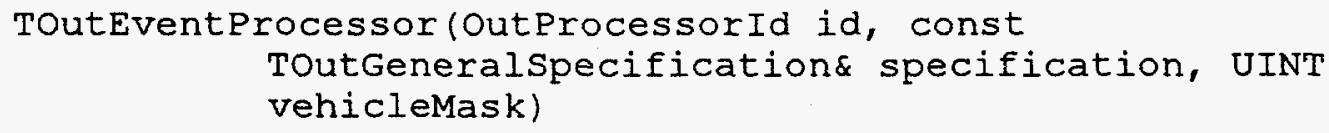

Construct an event processor.

EProcessorType GetProcessorType() const

Return the processor type.

virtual void Recordoutput (REAL time)

Begin recording output for this time step.

virtual void RecordVehicle()

Finish recording output for a vehicle. 
TOutObserver\& GetVehicleobserver()

const Toutobserver\& GetVehicleobserver() const

Return the vehicle observer.

void SetVehicleobserver (Toutobserver \& observer)

Define the vehicle observer.

UINT GetVehicleMask() const

Return the vehicle status mask.

\section{TOutSummaryProcessor}

An output summary processor collects statistics on the simulation. A summary processor has space and time observers and is connected to corresponding storages.

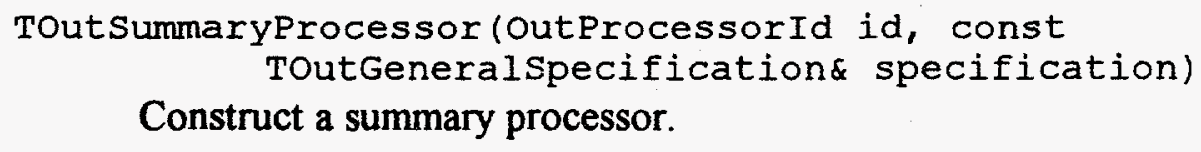

Construct a summary processor.

EProcessorType GetProcessorType() const

Return the processor type.

virtual void Recordoutput (REAI time)

Begin recording output for this time step.

virtual void RecordSpace (const TOutobserver\& observer)

Finish recording output for link space data.

virtual void RecordTime (const Toutobserver\& observer)

Finish recording output for link time data.

ObserverSet\& GetSpaceobservers()

const Observerset\& GetSpaceobservers() const

Return the space observers.

ObserverSet\& GetTimeobservers()

const ObserverSet\& GetTimeobservers() const

Return the time observers.

void AddSpaceobserver (TOutobserver\& observer)

Define a space observer.

void AddTimeobserver (TOutobserver \& observer)

Define a time observer.

void Removespaceobserver (TOutobserver\& observer)

Undefine a space observer. 
void RemoveTimeobserver (TOutobserver\& observer)

Undefine a time observer.

\section{TOutObserver}

An observer converts data from the object to which it is attached into the generic form understood by the output subsystem. An observer has an id and an output record. The class keeps track of the next available observer id.

TOutobserver (OutobserverId)

Construct an observer.

virtual void Observe(const void* object, const ToutProcessor\& processor)

An observer has an observe function for noting the values of data members of interest in the observed object. This virtual function in the basic representation must be overridden in the view.

OutObserverId GetId()

const OutobserverId GetId() const

Return the observer's id.

ToutRecord\& GetRecord()

const ToutRecord\& GetRecord() const

Return the associated record.

static OutobserverId GetNextId()

Return the next unused observer id.

void setTime (REAL time)

Define the record's time.

11. TOutVehicleObserver

A vehicle observer observes data related to vehicles.

ToutVehicleviseiver (Outobservertd id)

Construct a vehicle observer.

void SetId(UINT vehicle)

Define the vehicle's id.

void SetLink (NetLinkId link)

Define the id of the link the vehicle is on.

void SetLane (NetLaneNumber lane)

Define the lane number the vehicle is on.

void SetDistance (REAL distance)

Define the vehicle's distance from the node. 
void SetNode (NetNodeId node)

Define the id of the node from which the distance is measured from.

void SetVelocity (REAL velocity)

Define the vehicle's velocity.

void Setstatus (BYTE status)

Define the vehicle's status.

12. TOutNodeEvolutionObserver

A node evolution observer observes evolving data related to nodes.

TOutNodeEvolutionobserver (OutObserverId id)

Construct a node evolution observer.

13. TOutLinkEvolutionObserver

A link evolution observer observes evolving data related to links.

TOutLinkEvolutionobserver (OutobserverId id)

Construct a link evolution observer.

\section{TOutSignalCoordinatorEvolutionObserver}

A signal coordinator evolution observer observes evolving data related to signal coordinators.

TOutSignalCoordinatorEvolutionobserver (OutobserverId id)

Construct a signal coordinator evolution observer.

15. TOutSignalizedControlObserver

A signalized control observer observes data related to signals.

TOutSignalizedControlobserver (OutobserverId id)

Construct a signalized control observer

void SetLink(NetLinkId link)

Define the id of the link entering the intersection.

void SetLane (NetLaneNumber lane)

Define the lane number of the lane entering the intersection.

void SetNode (NetNodeId node)

Define the id of the node associated with the intersection.

void SetSignal (TNetTrafficControl: :ETrafficControl state)

Define the current signal state. 


\section{TOutIntersectionObserver}

A intersection observer observes data related to intersections.

TOutIntersectionobserver (OutobserverId id)

Construct an intersection observer.

void SetId(UINT vehicle)

Define the vehicle's id.

void SetLink (NetLinkId link)

Define the id of the link the vehicle entered from.

void SetLane (NetLaneNumber lane)

Define the lane number the vehicle entered from.

void SetNode (NetNodeId node)

Define the id of the node the intersection is associated with.

void SetoIndex (BYTE index)

Define the index of the vehicle's position in the queue.

\section{TOutLinkSpaceObserver}

A link space observer summarizes vehicle spatial data on a link. Each observer has a link length, a box length, and box data.

TOutLinkSpaceobserver (OutobserverId id)

Construct a link space observer.

bool IsInitialized() const

Return whether the observer has been initialized.

void ClearBoxData()

Clear the box data for the observer.

void SetLink (NetLinkId id)

Set the link id.

void SetNode (NetNodeId id)

Set the departure node id.

void setLengths (REAL linkLength, REAL boxLength, REAL celllength)

Set the link, box, and cell lengths.

void AddVehicle (REAL distance, REAL velocity)

Add a vehicle. 
void Reportobservations (TOutProcessor processor)

Report the observations to the specified processor.

\section{TOutLinkTimeObserver}

A link time observer records vehicle travel times on a link. Each link time observer has a vehicle count, a total of travel times, and a total of travel time squares.

TOutLinkTimeobserver (OutObserverId id)

Construct a link time observer.

void AddVehicle (REAL time)

Add a vehicle.

void ClearData()

Clear the data for the observer.

void SetLink (NetLinkId id)

Set the link id.

void SetNode (NetNodeId id)

Set the departure node id.

void Reportobservations (TOutProcessor\& processor)

Report the observations to the specified processor.

\section{TOutRetriever}

An output retriever acts as an interface to coordinate the retrieval of data stored in a simulation. Each retriever has a general specification and may refer to a network.

virtual void Retrieve()

Perform the retrieval.

ToutRetriever(const TOutGeneralspecification\& specification, const TNetNetwork* network = NULL)

Construct a retriever based on the given specification and network.

ToutGeneralspecification\& GetGeneralspecification()

const ToutGeneralspecification\& GetGeneralspecification() const

Return the general specification.

const TNetNetwork* GetNetwork() const

Return the network, if any.

void BasicRetrieve (Toutstorage\& storage, TOutWriter\& writer, bool sort, bool filter = TRUE)

Retrieve data from the specified storage and put it in the specified writer, sorting it if indicated, and performing time and space filtering if indicated. 


\section{TOutEvolutionRetriever}

An evolution retriever gets specific trajectory data from storage and coordinates its conversion and filtering. The retriever is connected to a vehicle storage, an intersection storage, and a signal storage.

TOutEvolutionRetriever(const TOutstorage: : HostSet\& hosts, const ToutGeneralspecification\& specification, const TNetNetwork* network = NULL)

Construct a reader for the specified hosts, given specification, and network.

void Retrieve(ToutWriter\& vehicleWriter, TOutWriter\& intersectionwriter, Toutwriter signalWriter, bool sort $=$ TRUE)

Perform the retrieval on the specified writers.

\section{TOutEventRetriever}

An event retriever gets specific event data from storage and coordinates its conversion and filtering. The retriever is connected to a vehicle storage.

TOutEventRetriever(const Toutstorage: : HostSet\& hosts, const ToutGeneralspecification\& specification, const TNetNetwork ${ }^{\star}$ network = NULL)

Construct a reader for the specified hosts, given specification, and network.

void Retrieve (TOutwriter\& vehiclewriter, bool sort $=$ TRUE)

Perform the retrieval on the specified writers.

\section{TOutSummaryRetriever}

An event retriever gets specific summary data from storage and coordinates its conversion and filtering. The retriever is connected to a space storage and a time storage.

ToutSummaryRetriever(const Toutstorage: :Hostset\& hosts, const ToutGeneralspecification\& specification, const TNetNetwork* network = NULI)

Construct a reader for the specified hosts, given specification, and network.

void Retrieve(ToutWriter\& spaceWriter, ToutWriter\& timeWriter, bool sort $=$ TRUE)

Perform the retrieval on the specified writers.

\section{TOutWriter}

An output writer provides an interface for the external writing of data from simulation output. The exception ToutwriterFailure is thrown if an operation fails.

Toutwriter()

Construct a writer. 


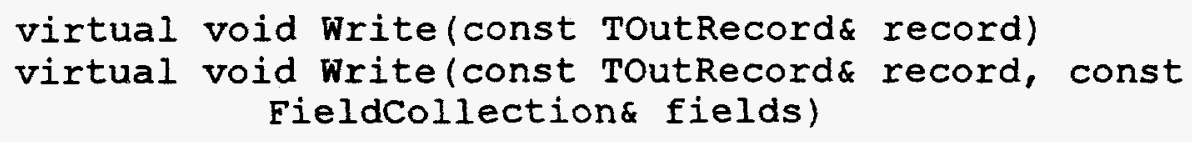

Write the specified record.

\section{TOutTextWriter}

A text writer puts simulation output data into a formatted text file. The exception ToutWriterfailure is thrown if an operation fails. Each text writer is connected to a file and has a delimiter. A text writer may have to include header information in its output.

ToutTextwriter(const string\& file, const string\& delimiter $=$ " (t", bool includeHeader = FALSE)

Open the specified file for writing, including the record header.

void Write(const ToutRecord\& record)

void Write(const TOutRecord\& record, const FieldCollection\& fields)

Write the specified record with fields in the given order.

\section{TOutStorage}

An output storage manages the distributed file system and isolates the rest of the simulation output objects from the details of the physical storage. Member functions throw the exception ToutstorageFailure when errors occur. The class has a local host name. Each instance has a file suffix, a root location, a basic name, and a file on each host.

static const long kBegin

static const long kEnd

Constants for seek positions.

enum Mode \{kRead, kWrite, kDelete\}

Storage modes: Use read mode for opening existing files, write mode for creating a new file, and delete mode for deleting existing files.

Toutstorage (const string \& root, const string\& name, Mode mode $=$ $k$ Read)

TOutStorage (const HostSet\& hosts, const string\& root, const string\& name, Mode mode $=$ kRead)

Connect the storage with the given root and basic name to the specified hosts.

const string\& GetRoot() const

Return the root name.

const string\& GetName() const

Return the basic name. 
Hostset GetHosts() const

Return the host names.

HostHandle GetHostHandle (const string\& host) const

Return the host handle.

long Getoffset() const

long Getoffset(Hosthandle host) const

long Getoffset (const string \& host) const

Return the current offset for the specified host file.

bool AtEnd() const

bool AtEnd (HostHandle host) const

bool AtEnd (const string \& host) const

Return whether an end-of-file has occurred for the specified host file.

void seek (long position = kBegin)

void Seek(Hosthandle host, long position = kBegin)

void Seek (const string\& host, long position $=\mathrm{kBegin}$ )

Position the specified host file to the given location.

void Write (const ToutRecord\& record)

void Write(HostHandle host, const ToutRecord\& record)

void Write (const string\& host, const ToutRecord\& record)

Write the given record on the specified host.

bool Read(ToutRecord\& record)

bool Read(const string\& host, ToutRecord\& record)

bool Read(HostHandle host, ToutRecord\& record)

Read the given record on the specified host. Return whether a record was available for reading.

void WriteHeader (const ToutRecord\& record)

void WriteHeader (const string\& host, const ToutRecord\& record)

void WriteHeader (HostHandle host, const ToutRecord\& record)

Write the given record header on the specified host.

bool ReadHeader (ToutRecord\& record)

bool ReadHeader (const string\& host, ToutRecord\& record)

bool ReadHeader (HostHandle host, ToutRecord\& record)

Read the given record header on the specified host. Return whether a record was available for reading.

void Flush()

Flush any pending operations.

\section{TOutRecord}

This class is used for storing the values of a collection of fields. Each record has a field map holding the current values of the fields. 
enum Type \{kNoType, kChar, kUnsignedChar, kshort, kUnsignedShort, kInt, kUnsignedInt, kLong, kUnsignedLong, kFloat, kDouble, kstring\}

Field types.

ToutRecord ()

Construct a record.

TOutRecord(const ToutRecord\& record)

Make a copy of the given record.

TOutRecord\& operator= (const TOutRecord\& record)

Make the record a copy of the given record.

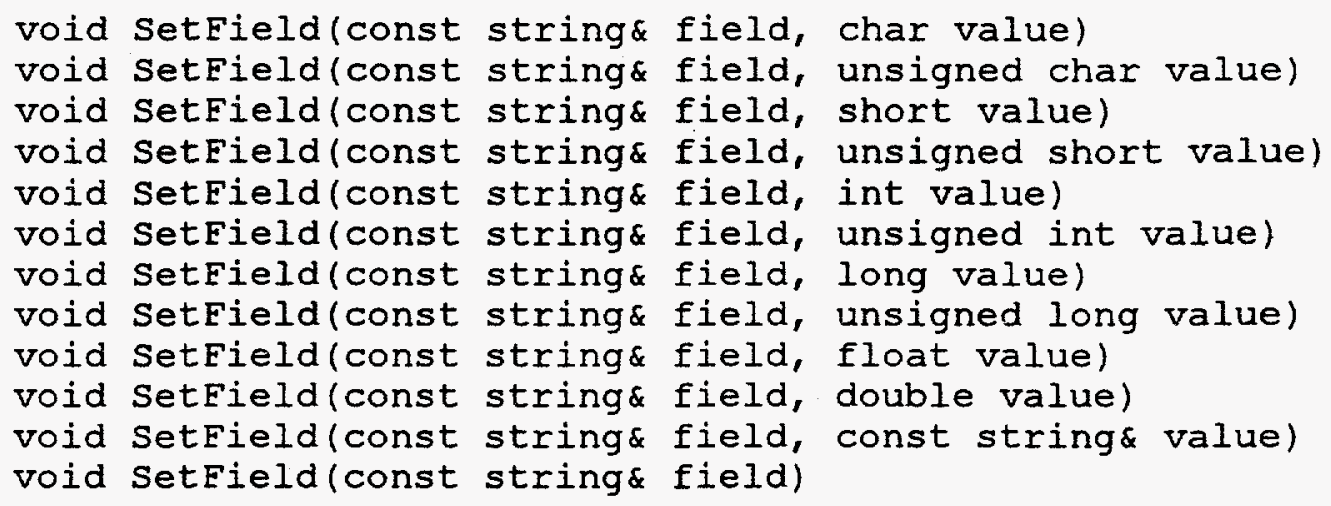

Set the value of the specified field.

void GetField(const string\& field, char\& value) const

void GetField(const string\& field, unsigned char\& value) const

void GetField(const string\& field, short\& value) const

void GetField(const string\& field, unsigned short\& value) const

void GetField(const string\& field, int\& value) const

void GetField(const string\& field, unsigned int\& value) const

void GetField(const string\& field, long\& value) const

void GetField(const string\& field, unsigned long\& value) const

void GetField(const string \& field, float\& value) const

void GetField(const string\& field, double\& value) const

void GetField(const string field, string\& value) const

Get the value of the specified field.

Type GetType (const string\& field) const

Return the type of the specified field.

FieldMap\& GetMap ()

const FieldMap\& GetMap() const

Return the map for the record.

FieldMapIterator GetIterator() const

Return an iterator for the record's map. 


\section{TOutException}

An output exception signals the failure of a simulation output subsystem function. Each exception has a message. Figure 9 shows the hierarchy of exception classes.

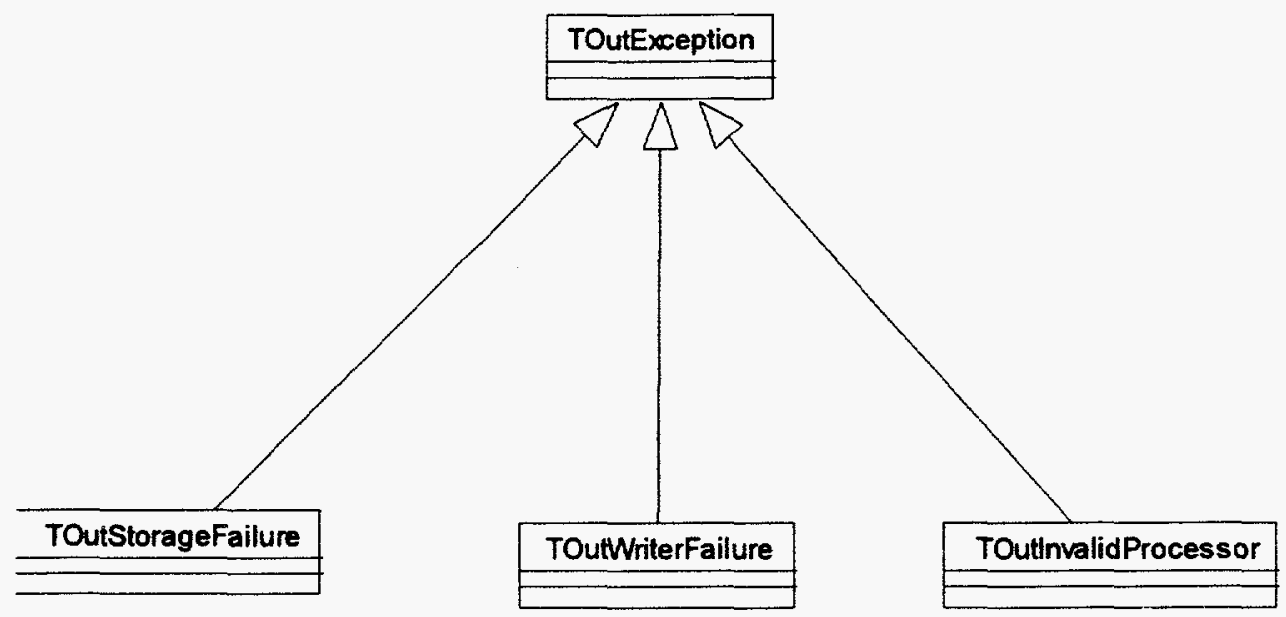

Figure 9. Exception hierarchy for the TRANSIMS simulation output subsystem (unified notation).

TOutException (const string\& message = "Simulation output error.")

Construct an exception with the specified message text.

ToutException(const ToutException\& exception)

Construct a copy of the specified exception.

TOutException\& operator=(const TOutException\& exception)

Make the exception a copy of the specified exception.

const string\& GetMessage() const

Return the message text for the exception.

class Toutstorage Failure

This exception is thrown when a storage operation fails.

class ToutwriterFailure

This exception is thrown when a writer operation fails.

class Tout InvalidProcessor

This exception is thrown when the processor type is invalid. 


\section{Implementation}

\section{A. C++ Libraries}

The Booch Components [RW 94] the provide $\mathrm{C}++$ container classes that the simulation output subsystem uses extensively. The DBtools.h++ [SL 95; Su 95] and Tools.h++ [Ke 94] libraries provide platform-independent data type and file system support, respectively. The subsystem also uses the standard C++ library [PI 95], the standard C library [P1 92], and the POSIX library [ $\mathrm{Ga} 95]$. All of these libraries compile on a wide variety of platforms (UNIX and otherwise).

\section{B. File System}

Although the simulation output subsystem runs on multiple computational nodes (CPNs), it stores output data locally (Figure 10) and thus does not require any communication between the CPNs. A unified view of the data is provided during data retrieval by accessing and collating the data on multiple remote disks (Figure 11).

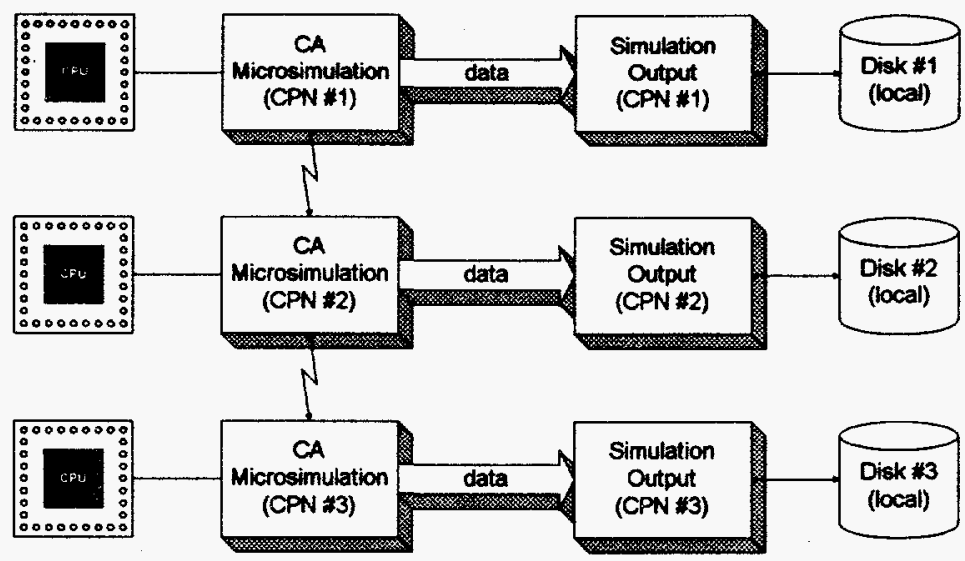

Figure 10. Simulation output subsystem configuration for simultaneous data collection on multiple CPNs.

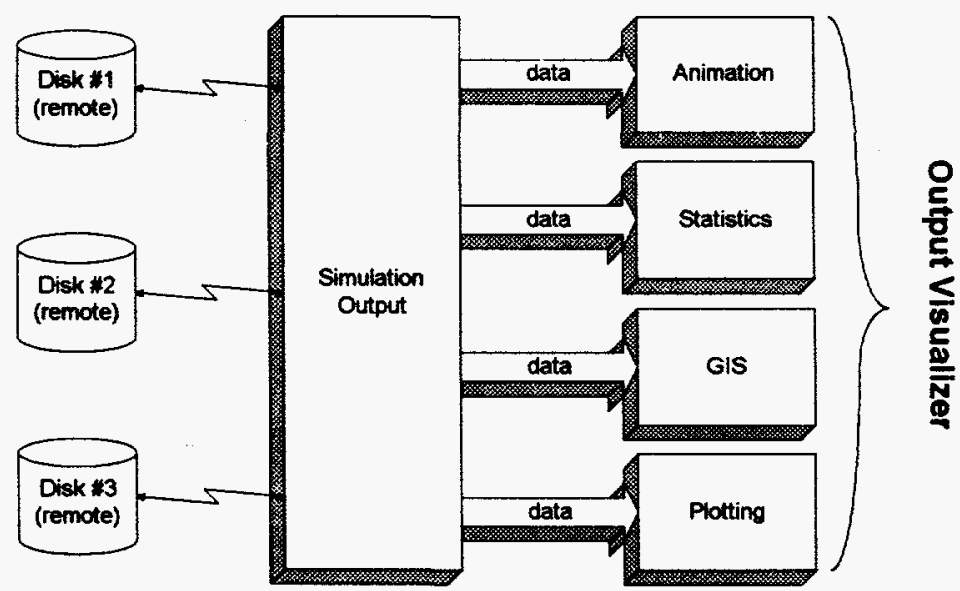

Figure 11. Simulation output subsystem configuration for data retrieval from multiple disks. 
The local-storage/remote-retrieval paradigm requires coordination between the microsimulation software and the postprocessing software. The simplest way to accomplish this is to have each CPN store data locally into a directory named "local" on each CPN-this will of course be a different physical disk for each CPN. Each of these "local" directories is given a different NFS (network file system) name (typically the name of the CPN) so that it can be accessed remotely. Figure 12 illustrates this scheme. Several other workable arrangements are possible, however.

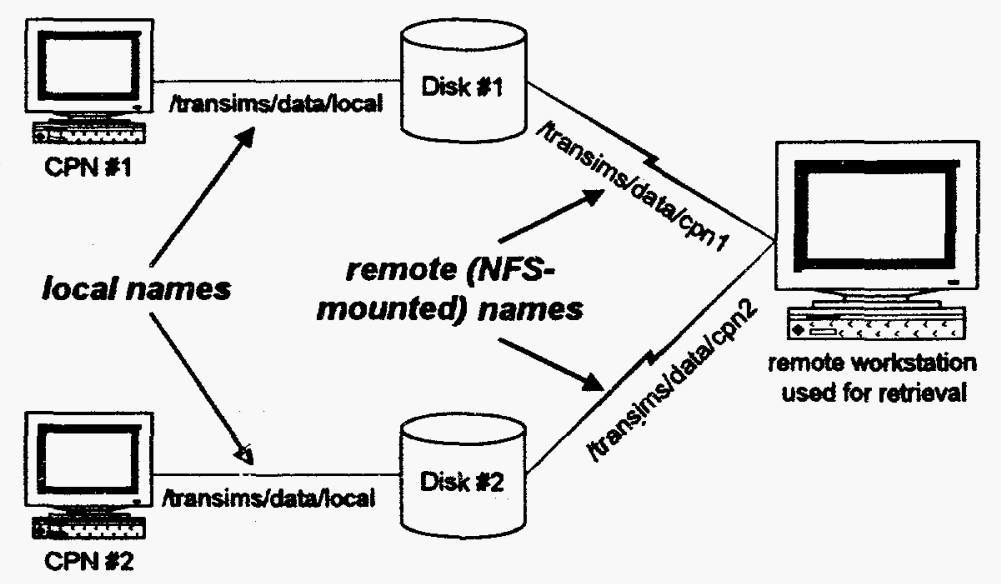

Figure 12. Typical naming and NFS-mounting scheme for simulation output directories.

\section{Integration into the Microsimulation}

The simulation output subsystem must be integrated into the microsimulation by subclassing the appropriate processor (TOut...Processor) and observer (TOut...Observer) classes and by providing a subclass of ToutFactory for creating instances of these subclasses. This mechanism allows a flexible yet tight coupling between the two subsystems without requiring the simulation output subsystem to be tailored to the specifics of the microsimulation. Figure 13 illustrates an example of how this subclassing can be implemented. 


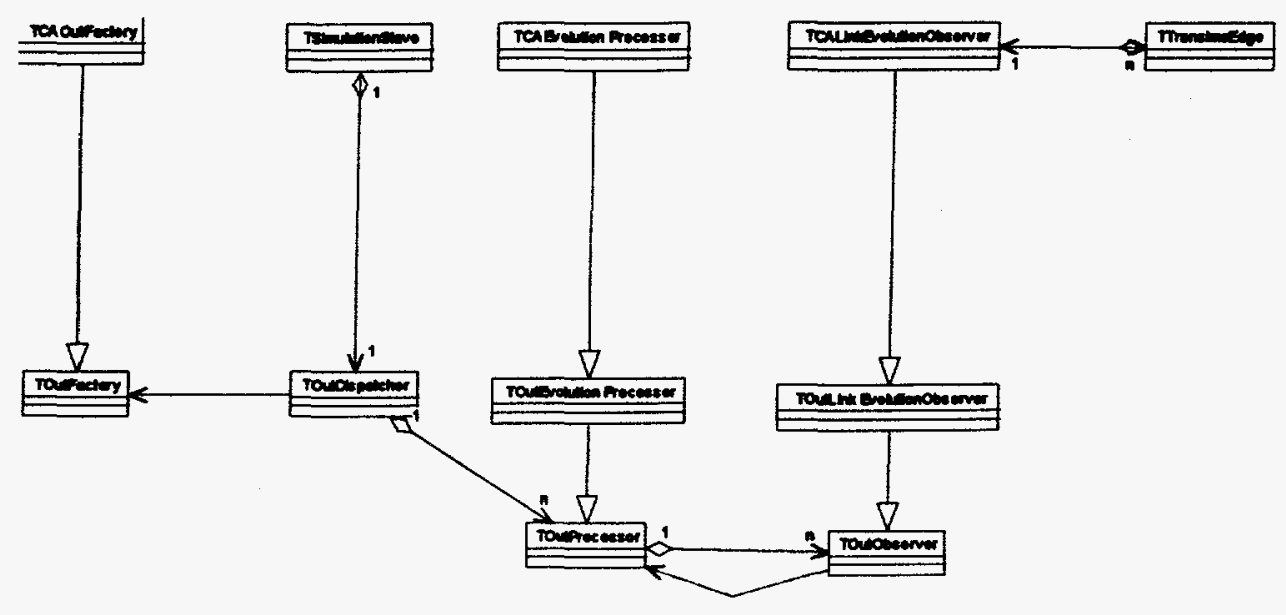

Figure 13. Erample of subclassing simulation output classes in a simulation (unified notation).

\section{Usage}

\section{A. Specification Formats}

This section describes the files that a user must currently prepare in order to use the simulation output subsystem in conjunction with the CA microsimulation.

The general specification output tables describe the general characteristics of the output that is to be collected during a CA run. These specifications are stored in the Oracle database management system prior to running the $\mathrm{CA}$, retrieved by the $\mathrm{CA}$ during execution, and used to guide the simulation output subsystem's collection and storage of data.

Tables may be created in Oracle by using the database functions of the Input Editor. Tables may also be created by using the database subsystem import utility, for which the usage is:

Import file

where file contains information and commands for the construction of one or more data tables. Import prints a brief help message if irvoked without a file. When constructing new tables, it is recommended that, when possible, an existing table description file which is similar to the desired new table description be copied and edited. This reduces the chances for errors in the SQL statements in the file.

Three tables are currently used to provide the general specification for the simulation output subsystem. Preparation of the files for each of these tables is described below.

\section{Output Specification}

The output specification table provides information about the time frame for collecting data and where the data should be stored. Table 1 defines the format for this table. The combination of ROOT and NAME must be unique among multiple simultaneous users of the 
subsystem, or else the output files will be overwritten. For example, evolutionary output might be collected on each CPN for vehicle, intersection, and signal data; the files created would be:

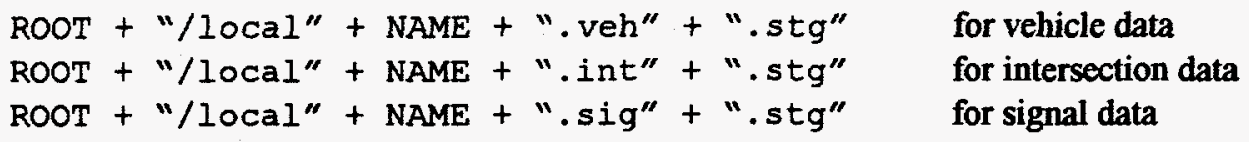

Typically, the directory ROOT + "/local" + NAME on each host is just a NFS (network file system) link to the physical directory ROOT + hostname + NAME; this allows both local and global views of the output directories (see Figure 12). Note that the output files must be deleted manually when they are no longer needed.

Table 1. Format for the output specification data table (an "Output Specification" data source in the database subsystem).

\begin{tabular}{|l|l|}
\hline Field & Interpretation \\
\hline ROOT & The directory where the output should be written. \\
\hline NAME & The output file name. \\
\hline PROCESSOR & $\begin{array}{l}\text { The type of processor: } \\
\text { - "Evolution": an evolution processor. } \\
\text { - "Event": an event processor. } \\
\text { - "Summary": a summary processor. }\end{array}$ \\
\hline TIMEMIN & The first time (in seconds from simulation start) at which to collect data. \\
\hline TIMEMAX & The last time (in seconds from simulation start) at which to collect data. \\
\hline TIMESTP & The frequency (in seconds) at which to report data (i.e., write it to disk). \\
\hline TIMESMP & The frequency (in seconds) at which to accumulate sample data. \\
\hline BOXIEN & The length of the boxes used for summary data. \\
\hline ABSCISSAMN & The minimum abscissa for which to collect data (currently ignored). \\
\hline ABSCISSAMX & The maximum abscissa for which to collect data (currently ignored). \\
\hline ORDINATEMN & The minimum ordinate for which to collect data (currently ignored). \\
\hline ORDINATEMX & The maximum ordinate for which to collect data (currently ignored). \\
\hline
\end{tabular}

An example output specification file is reproduced here:

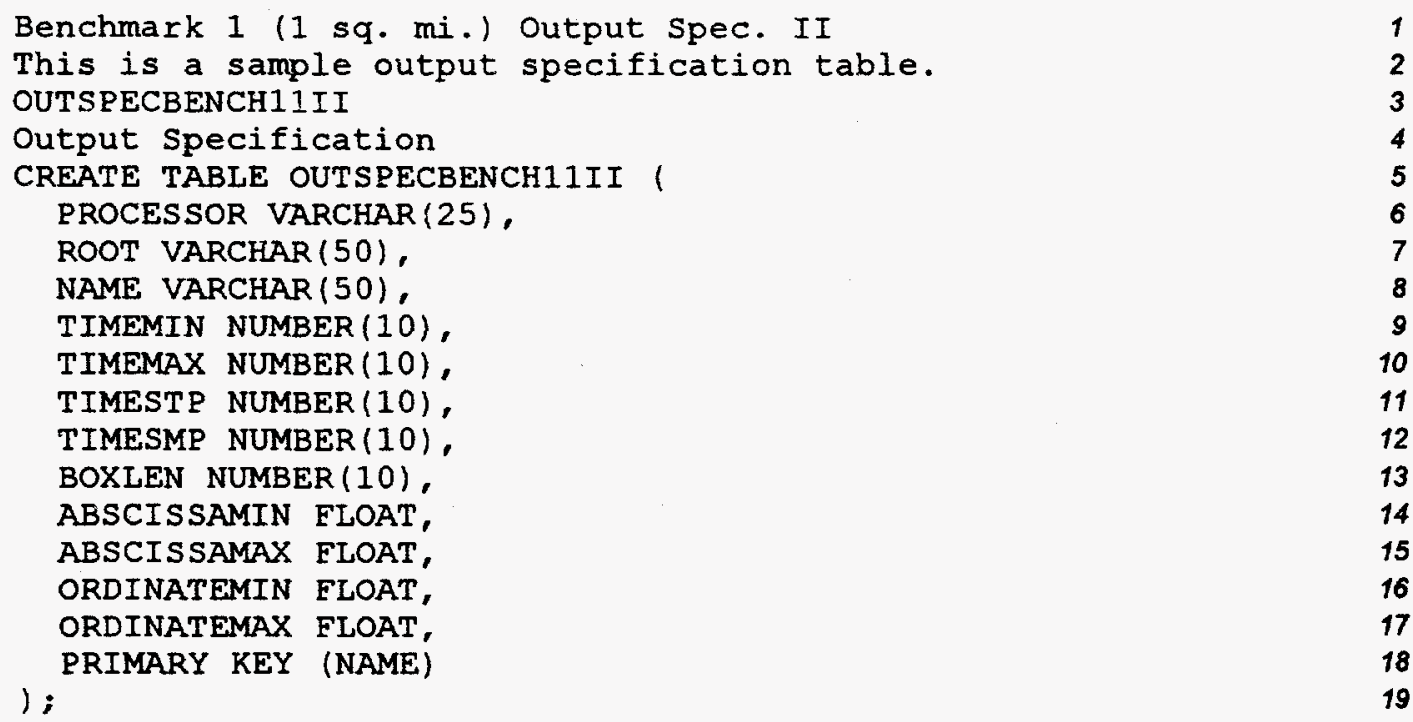




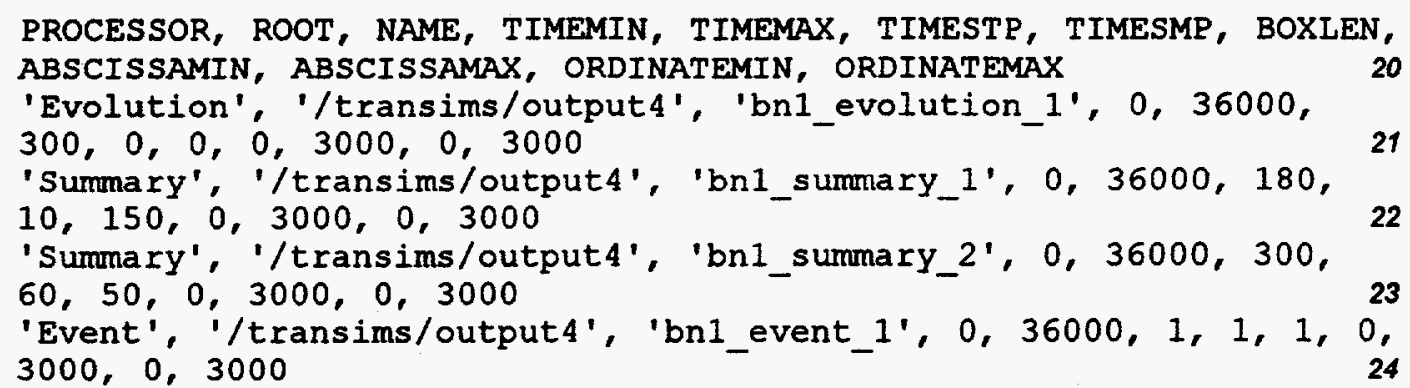

The first line of the file is the unique table name, with no more than 50 characters. The second line is a comment describing the table. Line 3 is a unique SQL table name. Line 4 is the data source name. Line 5 begins the SQL command for creating the table with the name specified in line 3. This command continues for fourteen additional lines and is terminated with a closing parenthesis and a semicolon. Line 20 names the columns whose values are specified in the same order and delimited by commas on lines 21 through 24 . One type of evolution data, two types of summary data, and one type of event data are specified for collection.

\section{Output Node Specification}

The output node specification table is used to specify the nodes at which data should be collected. Table 2 defines the format for this table. Until geographic filtering is supported this is the only way to indicate the nodes for which data collection occurs.

Table 2. Format for the output node specification data table (an "Output Node Specification" data source in the database subsystem).

\begin{tabular}{|l|l|}
\hline Field & Interpretation \\
\hline NAME & The output file name. \\
\hline NODE & The node id. \\
\hline
\end{tabular}

An example output node specification file is reproduced here:

$\begin{array}{lr}\text { Benchmark } 1 \text { (1 sq. mi.) Output Node Spec. II } & 1 \\ \text { This is a sample output node specification table. } \\ \text { OUTNODEBENCH11II } & 2 \\ \text { Output Node Specification } & 3 \\ \text { CREATE TABLE OUTNODEBENCH11II ( } & 4 \\ \text { NAME VARCHAR(50), } & 5 \\ \text { NODE NUMBER(10), } & 6 \\ \text { PRIMARY KEY (NAME, NODE) } & 7 \\ \text { J' } & 8 \\ \text { NAME, NODE } & 10 \\ \text { 'bn1_evolution_1',40006 } & 11 \\ \text { 'bn1_evolution_1',1793 } & 12 \\ \text { 'bn1_evolution_1',1808 } & 13 \\ \text { 'bn1_evolution_1',2413 } & 14 \\ \text { 'bn1_summary_2',2423 } & 15 \\ \text { 'bn1_summary_2',2424 } & 16 \\ \end{array}$


The first line of the file is the unique table name, with no more than 50 characters. The second line is a comment describing the table. Line 3 is a unique SQL table name. Line 4 is the data source name. Line 5 begins the SQL command for creating the table with the name specified in line 3. This command continues for four additional lines. Line 10 names the columns whose values are specified on lines 11 and following. Evolution data is collected on five nodes and summary data on two nodes.

\section{Output Link Specification}

The output link specification table is used to specify the links at which data should be collected. Table 3 defines the format for this table. Until geographic filtering is supported this is the only way to indicate the links for which data collection occurs.

Table 3. Format for the output link specification data table (an "Output Link Specification" data source in the database subsystem).

\begin{tabular}{|l|l|}
\hline Field & Interpretation \\
\hline NAME & The output file name. \\
\hline LINK & The link id. \\
\hline
\end{tabular}

An example output link specification file is reproduced here:

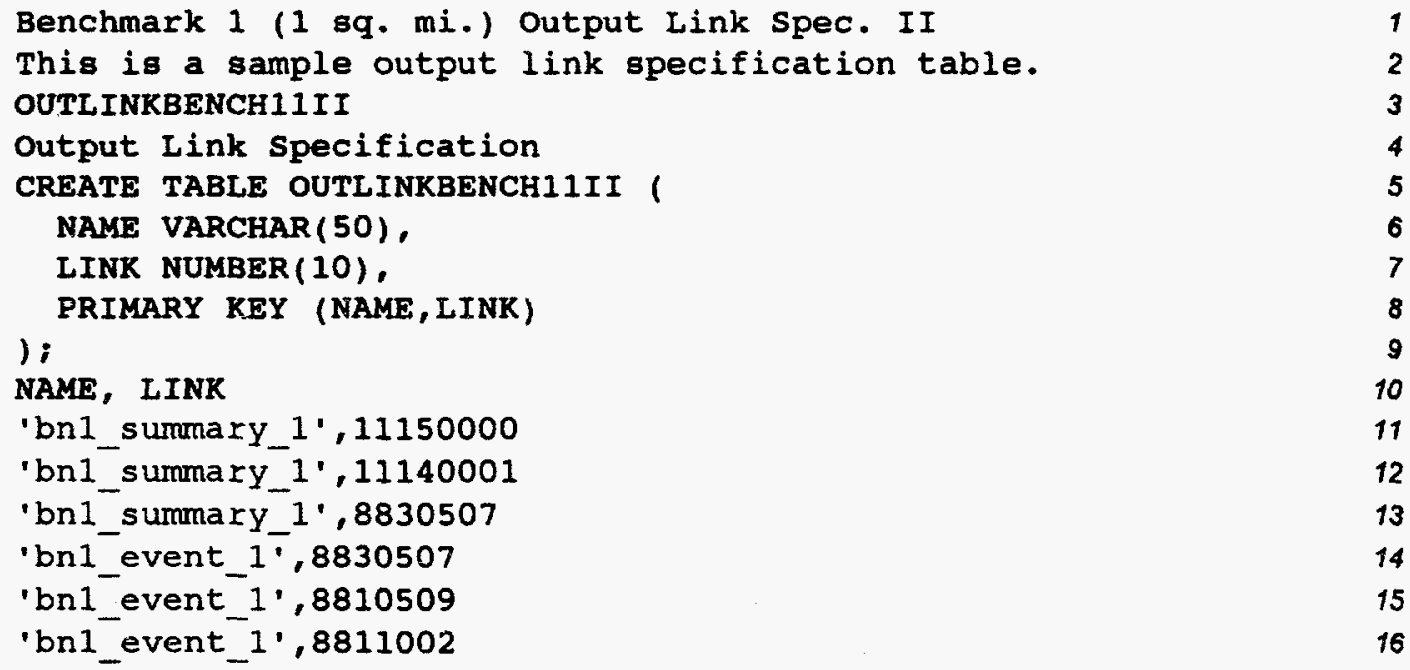

The first line of the file is the unique table name, with no more than 50 characters. The second line is a comment describing the table. Line 3 is a unique SQL table name. Line 4 is the data source name. Line 5 begins the SQL command for creating the table with the name specified in line 3 . This command continues for four additional lines. Line 10 names the variables whose values are specified on lines 11 and following. Summary data is collected on three links and event data on three links. 


\section{B. Data Retrieval}

The binary output stored in a distributed manner by the microsimulation is generally postprocessed to collect it into a single location. The DumpStorage utility may be used to postprocess the output into a text format for display or analysis. The usage is:

Dumpstorage outname root name hosts...

where outname is the destination file name, root is the root directory for the host subdirectories, name is the name of the storage file, and hosts... are the host subdirectory names. For example,

Dumpstorage bn1_summary_1.txt /transims/output4 bn1_summary_1.tim bach faure sous $\bar{a}$

collates the data from the files

/transims/output 4/bach/bn1 summary 1.tim.stg

/transims/output 4/faure/bn $\overline{1}$ summary 1. tim.stg

/transims/output 4/sousa/bn1_summary_1.tim.stg

into the file

. /bn1_summary_1.txt

in a tab-delimited ASCII text format.

\section{Output Formats}

\section{Evolution Data}

Vehicle evolution data files have the storage file suffix .veh.stg. Table 4 lists the fields present in such files; each record in the file represent a single vehicle. The data reporting start time, finish time, and reporting frequency are given by the output specification. The output specification also determines on which links the data is collected.

Table 4. Data format for vehicle evolution storage (.veh . stg) files.

\begin{tabular}{|c|c|}
\hline Field & Interpretation \\
\hline VEHICLE & The vehicle id. \\
\hline NODE & The node the vehicle was traveling away from. \\
\hline LINK & The link the vehicle was traveling on. \\
\hline LANE & The lane the vehicle was traveling on. \\
\hline TIME & The time the data was taken (in seconds from simulation start). \\
\hline DISTANCE & The distance (in meters) the vehicle was away from the node setback \\
\hline VELOCITY & The velocity (in meters per second) the vehicle was traveling. \\
\hline STATUS & $\begin{array}{l}\text { The vehicle status bits. } \\
\text { 1: The vehicle is lost. } \\
\text { - 2: The vehicle is at a dead end. } \\
\text { 4: The vehicle has just entered the study area. } \\
\text { 8: The vehicle has just exited the study area. } \\
\text { - 16: The vehicle is in the study area. } \\
\text { - 32: The vehicle has an invalid plan. }\end{array}$ \\
\hline
\end{tabular}


Intersection evolution data files have the storage file suffix . int.stg. Table 5 lists the fields present in such files; each record in the file represents a single vehicle. The data reporting start time, finish time, and reporting frequency are given by the output specification. The output specification also determines on which nodes the data is collected.

Table 5. Data format for intersection evolution storage (.int. $z \mathrm{tg})$ files.

\begin{tabular}{|l|l|}
\hline Field & Interpretation \\
\hline VEHICLE & The vehicle id. \\
\hline NODE & The node where the vehicle is located. \\
\hline TIME & The time the data was taken (in seconds from simulation start). \\
\hline LINK & The link the vehicle entered from. \\
\hline LANE & The lane the vehicle entered from. \\
\hline QINDEX & The vehicle position in the queue. \\
\hline
\end{tabular}

Signal evolution data files have the storage files suffix .sig.stg. Table 6 lists the fields present in such files; each record in the file represents a incoming lane at an intersection. The data reporting start time, finish time, and reporting frequency are given by the output specification. The output specification also determines on which nodes the data is collected.

Table 6. Data format for signal evolution storage (. $\mathrm{sig} . \mathrm{stg})$ files.

\begin{tabular}{|c|c|}
\hline Field & Interpretation \\
\hline NODE & The node where the signal is located. \\
\hline TIME & The time the data was taken (in seconds from simulation start). \\
\hline LNNK & The link entering the signal. \\
\hline LANE & The lane entering the signal. \\
\hline SIGNAL & $\begin{array}{l}\text { The type of control present: } \\
\text { - } 0: \text { None. } \\
\text { - } 1 \text { : Stop. } \\
\text { - } 2 \text { : Yield. } \\
\text { - } \text { 3: Wait. } \\
\text { - } 4: \text { Caution. } \\
\text { - } \text { 5: Permitted. } \\
\text { 6: Protected. }\end{array}$ \\
\hline
\end{tabular}

\section{Event Data}

Vehicle event data files have the storage file suffix .veh.stg. Table 7 lists the fields present in such files; each record in the file represents a single vehicle event. The data reporting start time, finish time, and reporting frequency are given by the output specification. This data is collected for all links-i.e., the output link specification is ignored. 
Table 7. Data format for vehicle event storage (.veh. stg) files.

\begin{tabular}{|c|c|}
\hline Field & Interpretation \\
\hline VEHICLE & The vehicle id. \\
\hline NODE & The node the vehicle was traveling away from. \\
\hline LINK & The link the vehicle was traveling on. \\
\hline LANE & The lane the vehicle was traveling on. \\
\hline TIME & The time the data was taken (in seconds from simulation start). \\
\hline DISTANCE & The distance (in meters) the vehicle was away from the node setback \\
\hline VELOCITY & The velocity (in meters per second) the vehicle was traveling. \\
\hline STATUS & $\begin{array}{l}\text { The vehicle status bits. } \\
\text { - 1: The vehicle is lost. } \\
\text { - } 2: \text { The vehicle is at a dead end. } \\
\text { 4: The vehicle has just entered the study area. } \\
\text { - } 8 \text { : The vehicle has just exited the study area. } \\
\text { 16: The vehicle is in the study area. } \\
\text { - } 32: \text { The vehicle has an invalid plan. }\end{array}$ \\
\hline
\end{tabular}

\section{Summary Data}

Link space summary data files have the storage file suffix .spa.stg. Table 8 lists the fields present in such files; each record in the file represents the summary for a single "box" on a link. If there is a short box, it is at the beginning of the link. The beginning distance of the box, which is not written in the file, is the ending distance of the box minus the box size. The data reporting start time, finish time, the sampling frequency, the data reporting frequency, and the box size are given by the output specification. The output specification also determines on which links the data is collected. Note that no data is reported at the reporting start time. Also, there may be two entries for links that are split by a CPN boundary; when this happens, it is necessary to add the respective COUNT and SUM entries for the duplicate box records.

Table 8. Data format for link space summary storage (. spa. s tg) files.

\begin{tabular}{|l|l|}
\hline Field & Interpretation \\
\hline LINK & The link being reported. \\
\hline NODE & The node from which vehicles were traveling. \\
\hline DISTANCE & The ending distance of the box (in meters) from the node setback \\
\hline TIME & The time the data was taken (in seconds from simulation start). \\
\hline COUNT & The number of vehicles in the box. \\
\hline SUM & The sum of the vehicle velocities (in meters per second) in the box. \\
\hline
\end{tabular}

Link time summary data files have the storage file suffix .tim.stg. Table 9 lists the fields present in such files; each record in the file represents the summary for a single direction of a link. The data reporting start time, finish time, and reporting frequency are given by the output specification. The output specification also determines on which links the data is collected. Note that no data is reported at the reporting start time. Also, there may be two entries for links that are split by a CPN boundary; when this happens, it is necessary to add the respective COUNT, SUM, and SUMSQUARES entries for the duplicate link records. 
Table 9. Data format for link time summary storage (.tim. stg) files.

\begin{tabular}{|l|l|}
\hline Field & Interpretation \\
\hline LINK & The link being reported. \\
\hline NODE & The node from which vehicles were traveling. \\
\hline TIME & The time the data was taken (in seconds from simulation start). \\
\hline COUNT & The number of vehicles leaving the link. \\
\hline SUM & $\begin{array}{l}\text { The sum of the vehicle travel times (in seconds) for vehicles leaving the link. } \\
\text { The time spent in the previous intersection is included in this value. }\end{array}$ \\
\hline SUMSQUARES & $\begin{array}{l}\text { The sum of the vehicle travel time squares (in seconds squared) for vehicles } \\
\text { leaving the link. The time spent in the previous intersection is included in } \\
\text { this value. }\end{array}$ \\
\hline
\end{tabular}

\section{Example of Retrieval Using C++}

The following example shows how to access and retrieve evolution data using $\mathrm{C}++$ calls to the simulation output subsystem.

// Create a character buffer for lines from standard input. char line [1000];

// Get the file name for the vehicle text output file. cin.getline (line, 1000);

TOutWriter ${ }^{\star}$ vehicleWriter $=$ new ToutTextWriter(line, "\t", TRUE);

// Get the file name for the output text files.

cin.getline(line, 1000);

ToutWriter* intersectionWriter = new ToutTextWriter(line, "\t", TRUE);

cin.getline(line, 1000);

TOutWriter ${ }^{*}$ signalWriter = new TOutTextWriter(line, "\t", TRUE);

TDbDirectory directory (TDbDirectoryDescription("IOC-1"));

// Open the data sources.

TDbSource generalsource (directory,

directory.GetSource ("Output Specification")):

TDbSource nodesource (directory, directory.GetSource("Output Node Specification"));

TDbSource linksource(directory, directory.GetSource("Output Link Specification"));

// Get the data table names and open the data tables. cin.getline(line, 1000);

TDbTable generalTable (generalsource, generalsource. GetTable (Iine));

cin.getline(line, 1000);

TDbTable nodeTable (nodeSource, nodeSource.GetTable(line));

cin.getline (line, 1000);

TDbTable IinkTable(linkSource, Iinksource.GetTable(line));

// Create the specification.

TOutGeneralspecificationReader

reader (TOutSpecificationReader (generalTable,

reader. Reset (); nodeTable, linkTable)); 


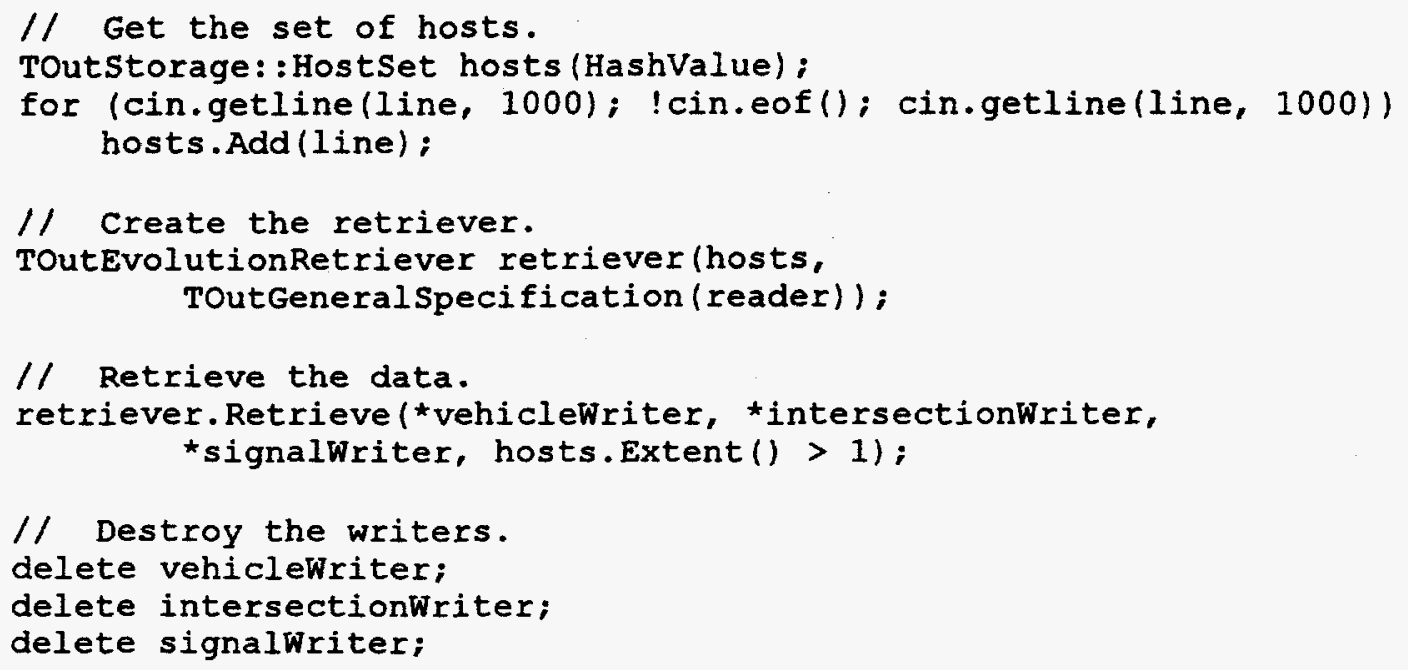

\section{B. Notes}

\section{Database Setup}

The CreateSources utility must be executed before the first use of the simulation output subsystem. This application registers the data sources in the database subsystem.

\section{Empty Storage Files}

The present implementation of the TOut...Retriever classes cannot handle input files of zero length. If no data is collected on a CPN, it is advisable to delete the empty storage file(s) created for that CPN.

\section{Future Work}

Future work planned for the TRANSIMS simulation output subsystem will focus on several areas:

- collecting new types of data such as turn counts, fundamental diagrams, and velocityacceleration histograms,

- enhancing the performance of the subsystem by supporting data compression, indexing, sorting, and filtering,

- improving the DumpStorage utility to provide more flexibility in exporting data, and

- eliminating the dependence on commercial products such as DBtools.ht+ and Tools.h+t.

\section{References}

[Ga 95] B. O. Gallmeister, POSIX.4: Programming for the Real World, (Sebastopol, California: O'Reilly \& Associates, 1995).

[Ke 94] T. Keffer, Tools.h++ Introduction and Reference Mamual, Version 6, (Corvallis, Oregon: Rogue Wave Software, 1994).

[PI 92] P. J. Plauger, The Standard C Library, (Englewood Cliffs, New Jersey: Prentice Hall, 1992). 
[P1 95] P. J. Plauger, The Draft Standard C ++ Library, (Englewood Cliffs, New Jersey: Prentice Hall, 1995).

[RW 94] Rogue Wave Software, The C++ Booch Components, Version 2.3, (Corvallis, Oregon: Rogue Wave Software, 1994).

[SL 95] S. Sulsky and K. L. Lohn, DBtools.h++ User's Guide and Tutorial, Version 1, (Corvallis, Oregon: Rogue Wave Software, 1995).

[Su 95] S. Sulsky, DBtools.h++ Class Reference, Version 1, (Corvallis, Oregon: Rogue Wave Software, 1995).

\section{APPENDIX: Booch Notation Diagrams}

Figure 14, Figure 15, Figure 16, and Figure 17 repeat the class diagrams of Figure 5, Figure 6, Figure 7, and Figure 8; Figure 18 repeats the exception hierarchy of Figure 9; and Figure 19 repeats the example of Figure 13 using Booch notation.

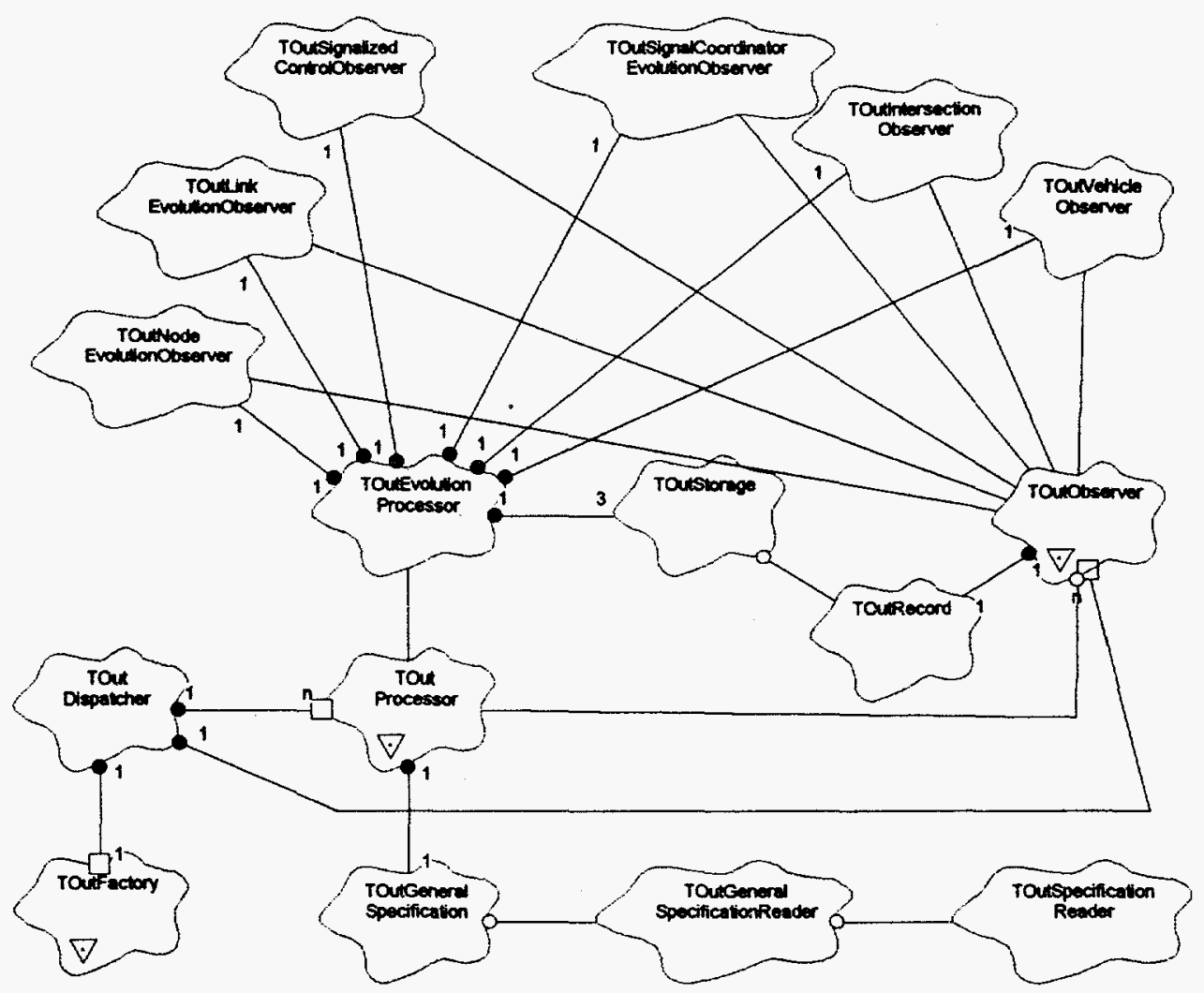

Figure 14. Class diagram for the TRANSIMS simulation output subsystem classes involved in evolution data collection (Booch notation). 


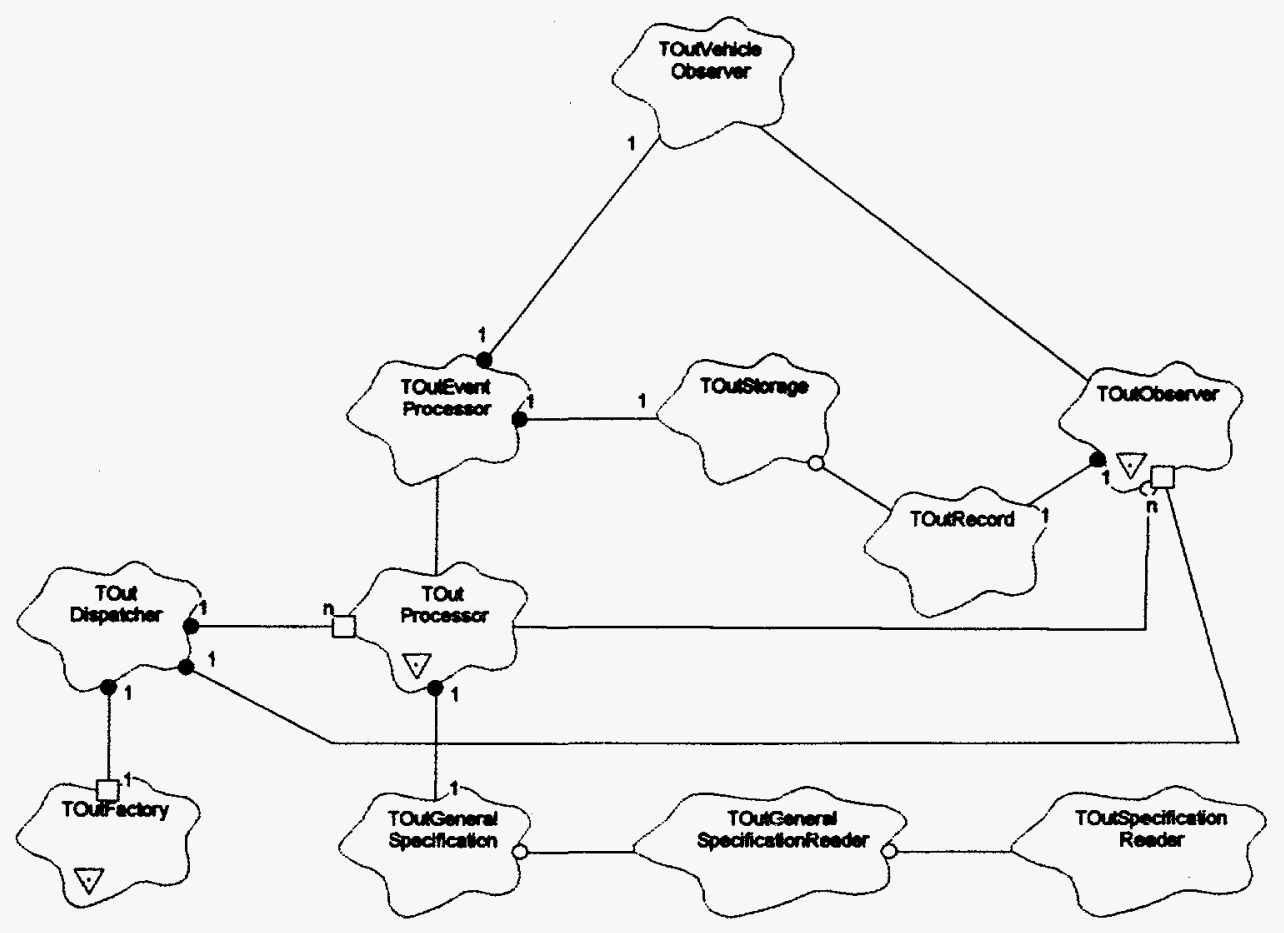

Figure 15. Class diagram for the TRANSIMS simulation output subsystem classes involved in event data collection (Booch notation).

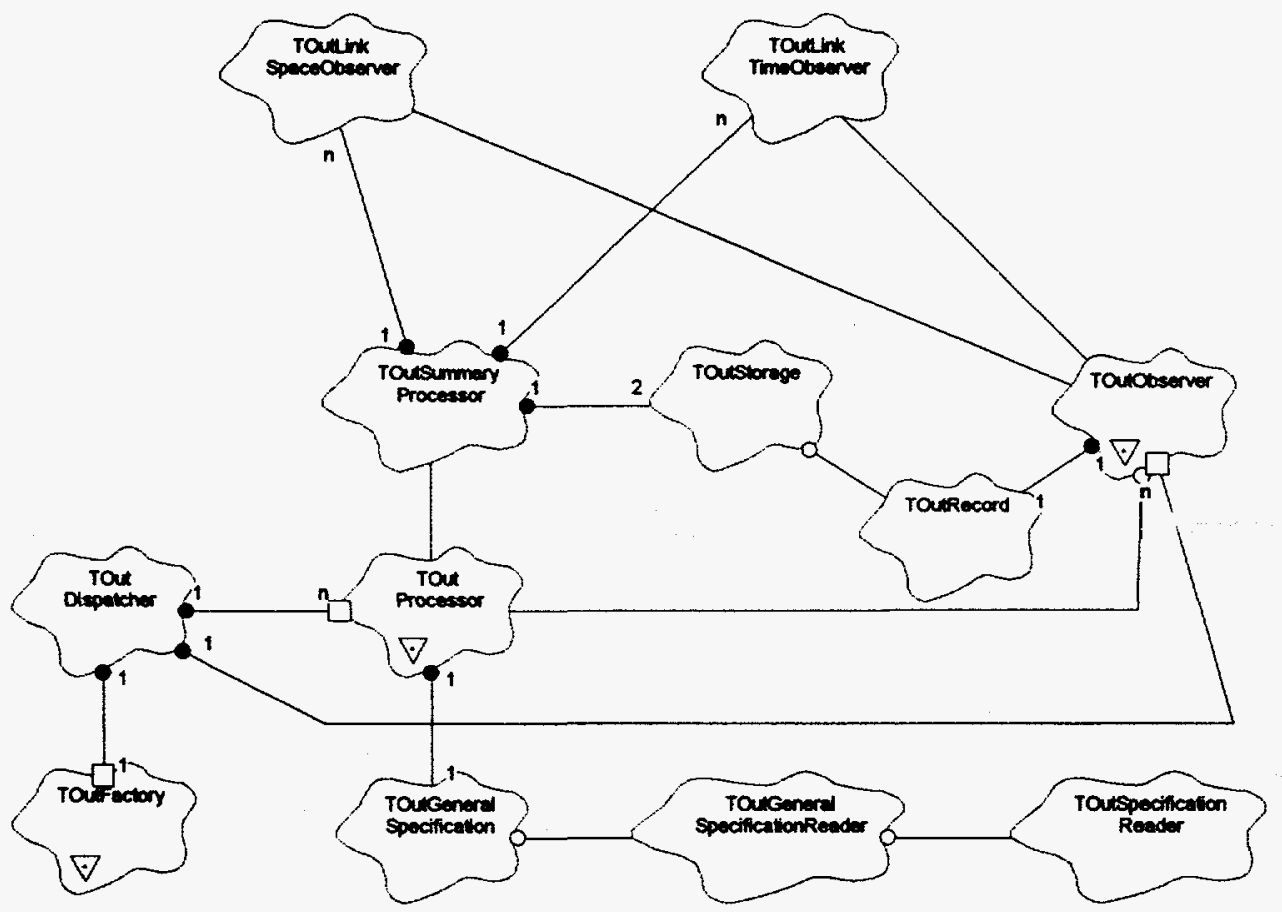

Figure 16. Class diagram for the TRANSIMS simulation output subsystem classes involved in summary data collection (Booch notation). 


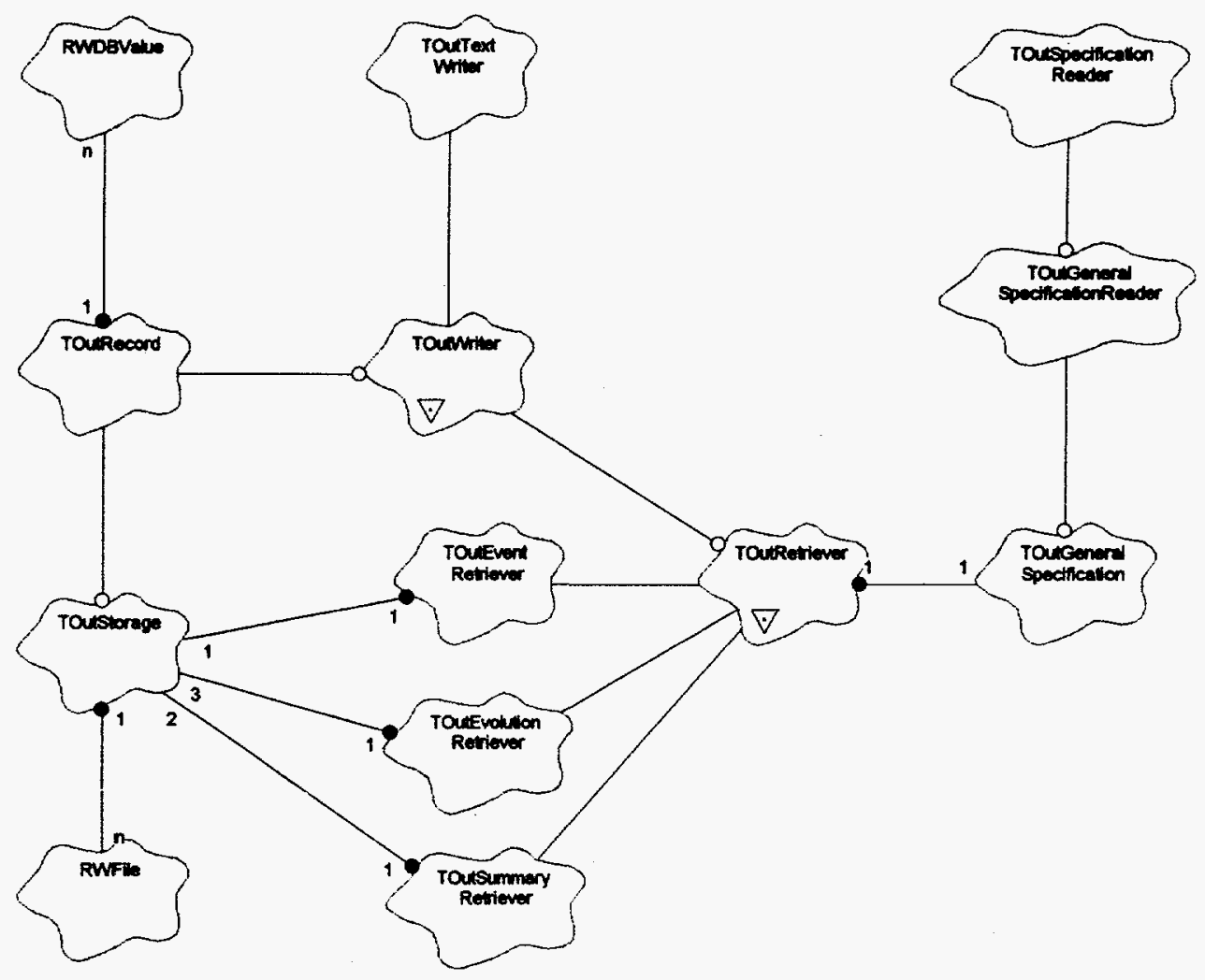

Figure 17. Class diagram for the TRANSIMS simulation output subsystem classes involved in data retrieval (Booch notation).

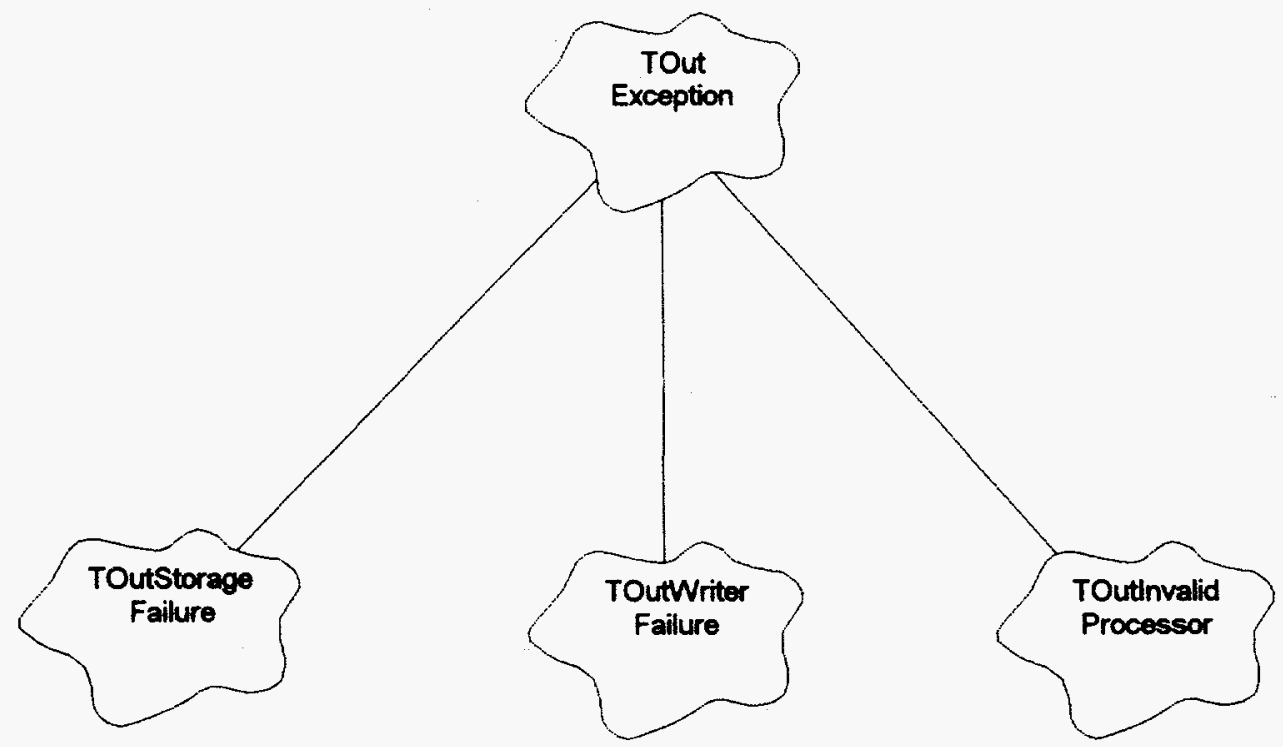

Figure 18. Exception hierarchy for the TRANSIMS simulation output subsystem (Booch notation). 


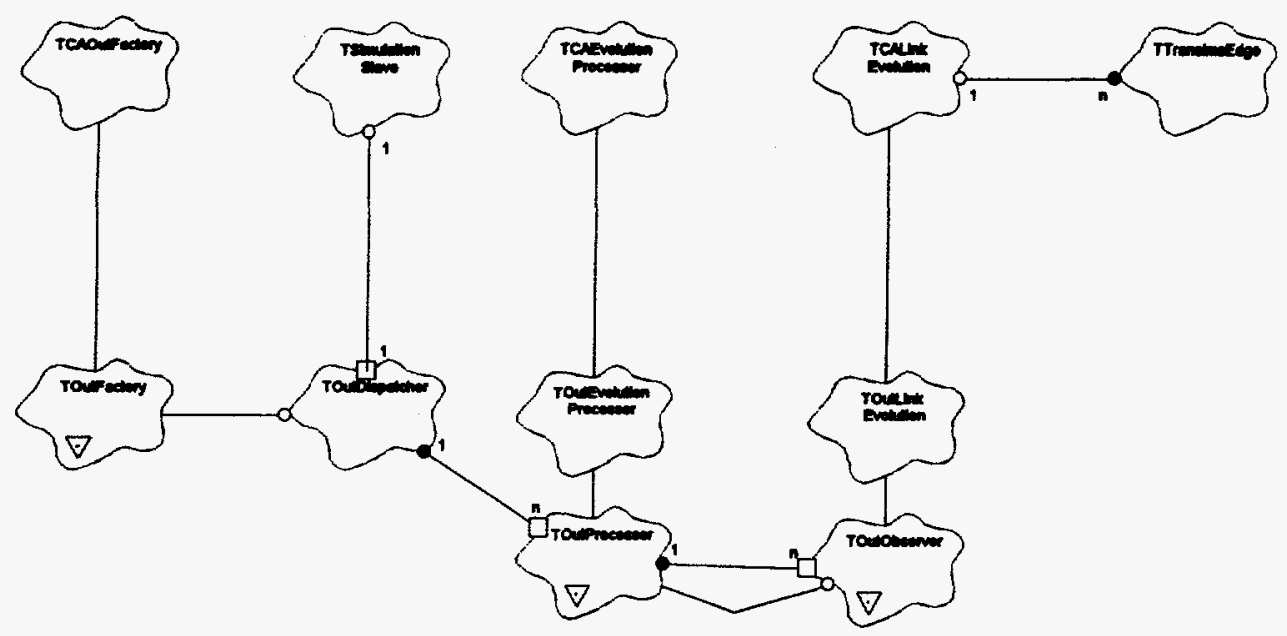

Figure 19. Example of subclassing simulation output classes in a simulation (Booch notation).

\section{APPENDIX: Source Code}

This appendix contains the complete $\mathrm{C}++$ source code for the simulation output subsystem classes.

\section{A. TOutDispatcher Class}

1. Dispatcher.h

$1 /$ Project: TRANSIMS

// Subsystem: Simulation Output

// SRCSfile: Dispatcher.h,v \$

// SRevision: 0.5 \$

// \$Date: 1996/06/19 17:07:17\$

// \$State: Stab \$

// SAuthor: bwb \$

// U.S. Government Copyright 1995

// All right reserved

\# ifndef TRANSIMS OUT DISPATCHER

\#define TRANSIMS_OUT_DISPATCHER

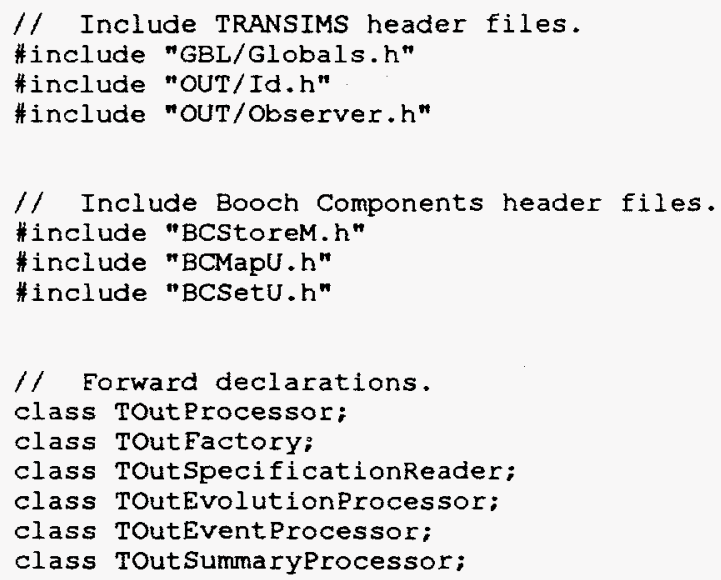


// objects and supervises the transfer of data.

class ToutDispatcher

1

public:

// Type definitions.

typedef BC TUnboundedMap<OutProcessorId, TOutProcessor*, 10U, BC CManaged> Processormap;

typedef BC_TMapActiveIterator<OutProcessorId, TOutProcessor*> ProcessorMapIterator:

typedef BC_TUnboundedMap<OutObserverId, TOutObserver*, 10U, BC_CManaged> ObserverMap;

typedef BC TMapActiveIterator<OutObserverId, TOutObserver*> ObserverMapIterator;

typedef BC TUnboundedSet<TOutEvolutionProcessor*, 10U, BC CManaged> EvölutionProcessorset;

typedef BC TSetActiveIterator<ToutEvolutionProcessor ${ }^{\star}>$ EvolutionProcessorsetIterator;

typedef BC_TUnboundedSet<TOutEventProcessor*, 10U, BC_CManaged> EventProcessorset:

typedef BC_TSetActiveIterator<TOutEventProcessor*> Event ProcessorsetIterator;

typedef BC TUnboundedSet<TOutSummaryProcessor*, 10U, BC CManaged> SummaryProcessorset:

typedef BC TSetActiveIterator<TOutSummaryProcessor*> SummaryProcessorsetIterator;

$/ /$ Observer types.

enum EObserverType (kNodeEvolutionobserver, klinkEvolutionobserver, kVehicleobserver, kIntersectionobserver, kSignalCoordinatorEvolutionobserver, kSignalizedControlobserver, kLinkspaceobserver, kLinkTimeObserver\};

// Construct an output dispatcher.

ToutDispatcher(TOutspecificationReader\& reader, ToutFactory\& factory);

// Destruct an output dispatcher.

-TOutDispatcher():

// Return whether two output dispatchers are the same.

bool operator $=$ (const TOutDispatcher\& dispatcher) const

bool operator!=(const ToutDispatcher\& dispatcher) const:

// Begin output recording for this time step.

void Recordoutput (REAL timel;

// Return the processors.

ProcessorMap\& GetProcessors (1)

const ProcessorMap\& GetProcessors() const

/1 Return the observers.

ObserverMap\& GetObservers();

const ObserverMap\& Getobservers () const;

i/ Return the evolution processors.

EvolutionProcessorset\& GetEvolutionProcessors();

const EvolutionProcessorSet\& GetEvolutionProcessors () const;

$1 /$ Return the event processors.

EventProcessorSet\& GetEventProcessors (1);

const EventProcessorSet GetEventProcessors() const;

// Return the summary processors.

SummaryProcessorset\& GetSummaryProcessors();

const SummaryProcessorSet\& GetSummaryProcessors() const;

// Define the node observers.

void SetNodeObservers (TOutObserver: :ObserverMap\& observers) :

// Define the link observers.

void SetLinkObservers (TOutObserver: : ObserverMap\& observers);

// Define the vehicle observers.

void SetVehicleobservers (TOutObserver: :ObserverMap\& observers); 
// Define the intersection observers.

void SetIntersectionobservers (TOutObserver: :ObserverMap\& observers):

// Define the signal coordinator observers.

void SetSignalCoordinatorobservers (TOutObserver: :ObserverMap\& observers);

// Define the signalized control observers.

void SetSignalizedControlobservers (TOutObserver: :ObserverMap\& observers);

// Define the link space observers.

void SetLinkSpaceObservers (TOutObserver: :ObserverMapd observers);

// Define the link time observers.

void SetIinkTimeObservers (TOutObserver: : ObserverMap\& observers);

1/ Undefine the link space observers.

void ClearLinkSpaceObservers (TOutObserver::ObserverMap\& observers);

// Undefine the link time observers.

void ClearLinkTimeObservers (TOutObserver: :ObserverMap observers);

private:

// Do not allow dispatchers to be copied.

TOutDispatcher(const TOutDispatcher\&) \{\}

// Do not allow dispatchers to be assigned.

TOutDispatcher operator= (const TOutDispatcher\&) (return *this;)

// Each dispatcher has a processor map.

Processormap fProcessors;

// Each dispatcher has an observer map.

ObserverMap fobservers:

// Each dispatcher has evolution processors.

EvolutionProcessorset fEvolutionProcessors:

// Each dispatcher has event processors.

EventProcessorset fEventProcessors;

// Each dispatcher has summary processors.

SummaryProcessorset fSummaryProcessors;

// Each dispatcher remembers the factor it uses.

; ;

TOutFactory* fFactory;

\#endif // TRANSIMS_OUT_DISPATCHER

\section{Dispatcher.C}

$1 /$ Project: TRANSIMS

1/ Subsystem: Simulation Output

// SRCSfile: Dispatcher.C, $\$$

// SRevision: 0.5 \$

// SDate: 1996/06/19 17:06:25\$

$1 /$ \$state: stab \$

11 SAuthor: bwb \$

// U.S. Government Copyright 1995

// All right reserved

$/ /$ Include TRANSIMS header filles.

\#include "OUT/Dispatcher.h"

\#include "OuT/Processor.h"

\#include "OUT/Factory.h"

\#include "OUT/Exception.h"

"include "OUT/EvolutionProcessor.h"

\#include "OUT/EventProcessor.h"

\#include "OUT/SummaryProcessor.h"

\#include "OUT/LinkEvolutionobserver.h" 


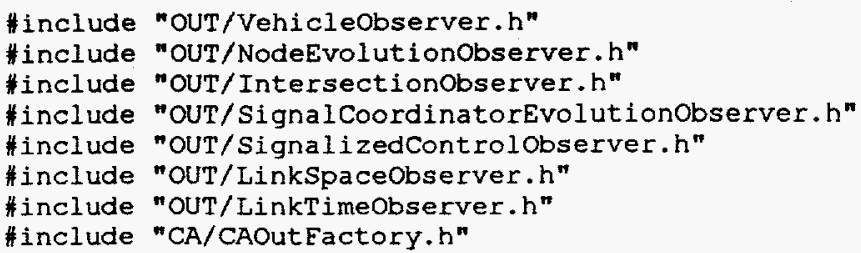

1/ Define the hash function for evolution processor sets.

static BC_Index EvolutionProcessorHashValue(TOutEvolutionProcessor* const\& proc)

// Define the hash function for event processor sets. static BC Index EventProcessorhashValue (TOutEventProcessor* const\& proc) i

// Construct an output dispatcher.

TOutDispatcher: :TOutDispatcher(TOutSpecificationReader\& reader, TOutFactory\& factory)

: fProcessors (ProcessorIdHashValue). fObservers (ObserverIdHashvalue), fEvolutionProcessors (EvolutionProcessorHashvalue), fEventProcessors (EventProcessorHashValue). fSumaryProcessors (SummaryProcessorHashvalue), fFactory ( \&actory)

TOutGeneralSpecificationReader genReader (reader):

for (genReader.Reset (); genReader.MoreSpecifications(); genReader.GetNextSpecification(1) (

if (genReader. GetProcessorType() == ToutGeneralspecificationReader: :kEvolutionfrocessor) (

TOutEvolutionProcessor $*$ proc $=$ factory. NewEvolutionProcessor (TOutProcessor: : GetNextId(), TOutGeneralSpecification (genReader)): fProcessors.Bind (proc->GetId (), proc); fEvolutionProcessors. Add (proc);

OutobserverId oid:

oid = kLinkEvolutionobserver;

if (fobservers.IsBound (oid) $==0$ ) ( TOutLinkEvolutionObserver* obs = factory.NewLinkEvolutionobserver(oid): fobservers. Bind (obs->GetId(), obs): 


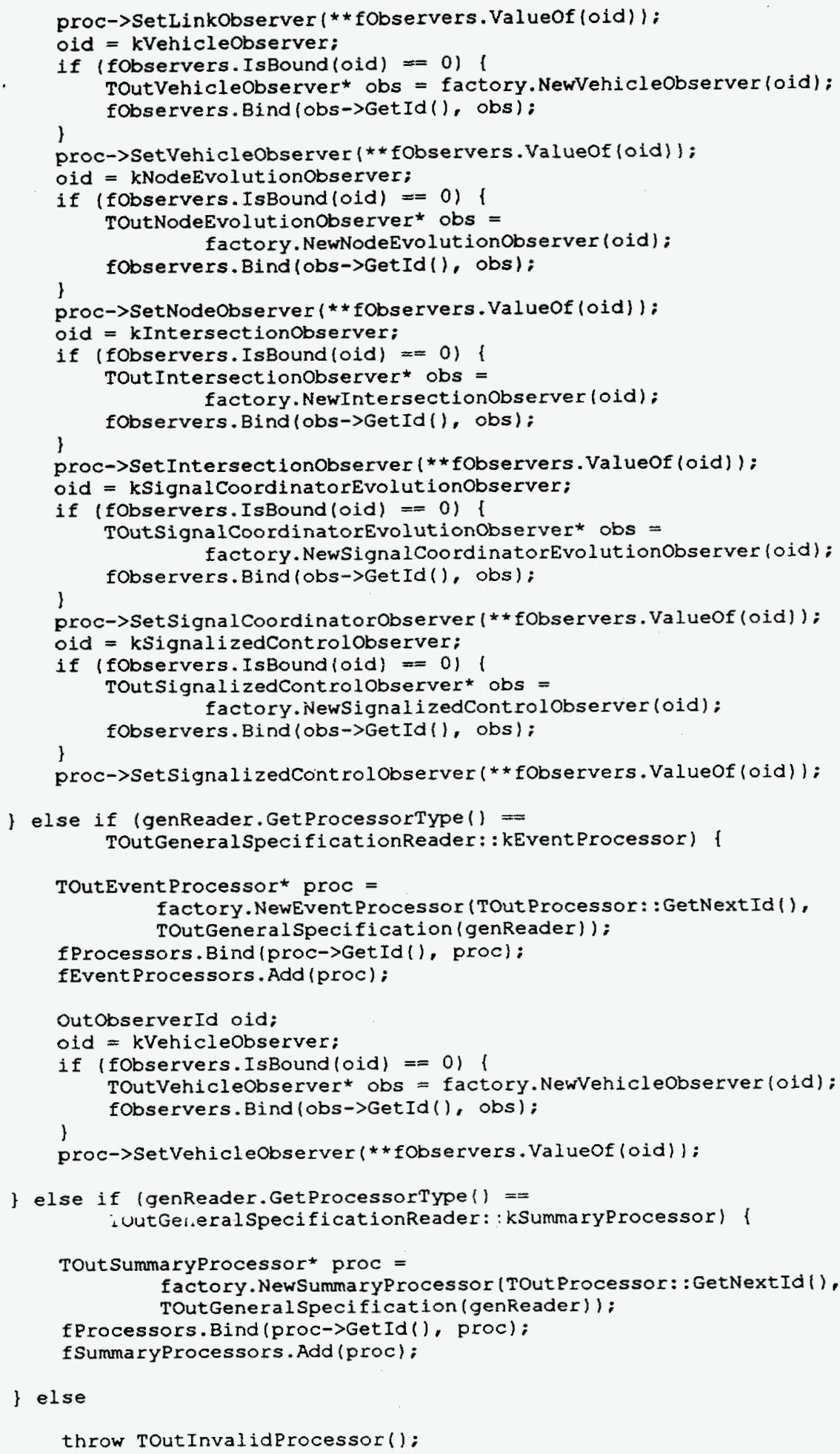




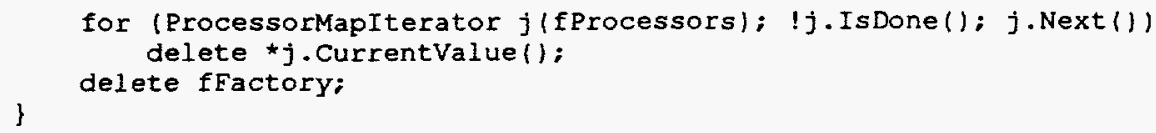




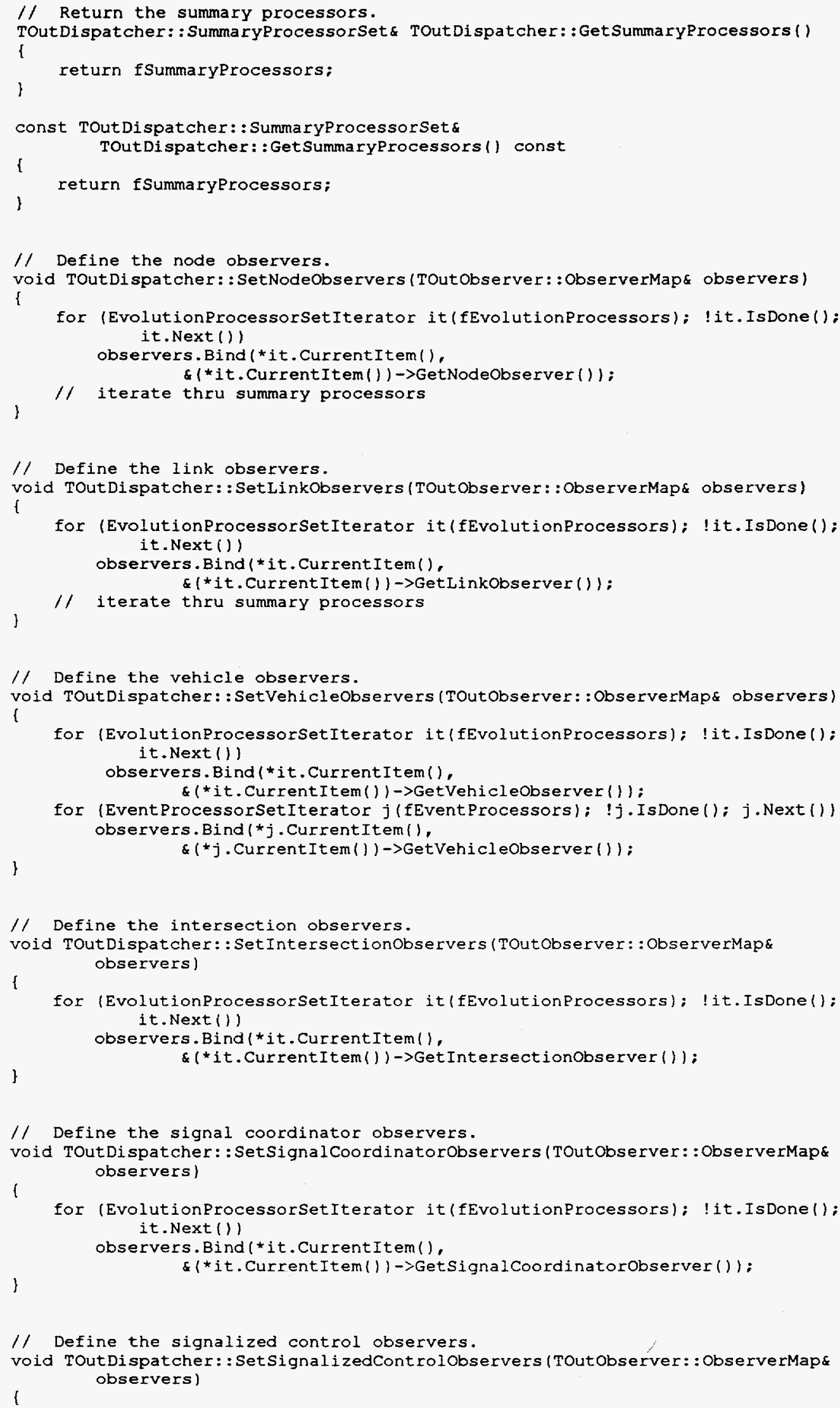


for (EvolutionProcessorsetIterator it(fEvolutionProcessors): ! it. IsDone(); it. Next ())

observers.Bind (*it. CurrentItem(), $\&(* i t$. CurrentItem( )) $\rightarrow$ GetSignalizedControlobserver ()):

\}

// Define a link space observers.

void TOutDispatcher: :SetLinkSpaceobservers (TOutObserver: : ObserverMap\& observers) I

TOutIinkSpaceobserver* observer =

fEactory->NewLinkSpaceObserver (TOutObserver: : GetNextId()):

for (SummaryProcessorsetIterator i (fSummaryProcessors); ! i. IsDone(); i. Next ()) (

observers.Bind (*i. CurrentItem(), observer):

(*i. CurrentItem ()) $\rightarrow$ AddSpaceobserver (*observer);

了

fobservers.Bind (observer->GetId(), observer);

// Define a link time observers.

void TOutDispatcher: : SethinkTimeobservers(TOutObserver: :ObserverMap\& observers) t

ToutLinkTimeobserver* observer =

fFactory->NewLinkTimeObserver (TOutObserver: :GetNextId ());

for (SummaryProcessorSetIterator i (fSummaryProcessors): ! i. IsDone(); i. Next ()) i

observers.Bind (*i. CurrentItem(), observer);

$(* i$. CurrentItem())->AddTimeobserver (*observer) :

\}

fObservers.Bind (observer->GetId (), observer);

1/ Undefine a link space observers.

void TOutDispatcher: : ClearLinkSpaceObservers (TOutObserver: : ObserverMap observers

bool deleted = EALSE;

for (TOutObserver::ObserverMapIterator i(observers); !i.IsDone(): i. Next ()) \{

ToutSummaryProcessor* processor $=$ (TOutSummaryProcessor ${ }^{*}$ )

*i. CurrentItem ():

Toutobserver* observer $=* i$. CurrentValue();

processor $\rightarrow$ RemoveSpaceobserver (*observer):

fobservers.Unbind (observer->GetId());

if (ldeleted) (//ISSUE(bwb): We may have to generalized this later. delete observer

\} deleted = TRUE;

observers. Clearl):

\}

(1) Undefine a link time observers.

void TOutDispatcher: :ClearLinkTimeobservers(TOutobserver: : ObserverMap\&

I observers)

bool deleted = EALSE;

for (TOutObserver:: ObserverMapIterator i (observers); ! $\mathfrak{i}$. IsDone (): i. Next ()) 1

TOutSummaryProcessor ${ }^{*}$ processor $=$ (TOutSummaryProcessor ${ }^{*}$ )

*i. CurrentItem()

TOutobserver* observer $=* i$. CurrentValue ():

processor $\rightarrow$ RemoveTimeobserver (*observer);

fobservers. Unbind (observer->GetId()):

if (!deleted) (//ISSUE (bwb): We may have to generalized this later.

delete observer:

)

deleted = TRUE;

observers.Clear(\};

1 


\section{B. TOutEventProcessor Class}

\section{EventProcessor.h}

$1 /$ Project: TRANSIMS

// Subsystem: Simulation Output

// \$RCSfile: Eventprocessor.h,v \$

// \$Revision: 1.2 \$

// \$Date: 1996/06/19 17:13:17 \$

// \$State: stab \$

// \$Author: bwb \$

// U.S. Government Copyright 1996

// All right reserved

\#ifndef TRANSIMS OUT EVENTPROCESSOR "define TRANSIMS_OUT_EVENTPROCESSOR

// Include TRANSIMS header files.

"include "OUT/Processor.h"

\#include "OuT/Storage. $h$ "

$1 /$ Forward declarations.

class Toutobserver;

$1 /$ An output event processor deals with conditions occuring in the simulation // such as vehicle entry, vehicle exit, and lost vehicles.

class TOutEventProcessor

1

: public TOutProcessor

public:

//. Construct a event processor.

TOutEventProcessor (OutProcessorId id, const TOutGeneralspecification specification, UINT vehicleMask);

$1 /$ Destruct a event processor

virtual TOutEventProcessor():

$1 /$ Return the processor type.

EProcessorType GetProcessorType() const;

// Begin recording output for this time step.

virtual void Recordoutput (REAL time);

// Finish recording output for a vehicle.

virtual void RecordVehiclel):

// Return the vehicle observer.

TOutObserver \&etVehicleobserver ():

const TOutObserver GetVehicleobserver() const;

// Define the vehicle observer.

void SetVehicleobserver(TOutobservers observer);

// Return the vehicle status mask.

UINT GetVehicleMask() const:

private:

// An event processor has a vehicle observer.

TOutobserver* fVehicleobserver:

// An event processor is connected to a vehicle storage.

TOutStorage fVehiclestorage;

$1 /$ An event processor has a vehicle status mask.

\};

UINT fVehicleMask; 
\#endif // TRANSIMS_OUT_EVENTPROCESSOR

\section{EventProcessor.C}

$1 /$ Project: TRANSIMS

1/ Subsystem: Simulation Output

// SRCSfile: EventProcessor.C,v $\$$

1/ SRevision: 1.2 \$

// SDate: 1996/06/19 17:12:02 \$

$1 /$ SState: Stab \$

// \$Author: bwb \$

// U.S. Government Copyright 1996

// All right reserved

// Include TRANSIMS header files.

\#include "OUT/EventProcessor.h"

\#include "OUT/Exception.h"

\#include "OUT/Observer.h"

\#include "OUT/Names.h"

// Construct an event processor.

TOutEventProcessor: : TOutEventProcessor (OutProcessorId id, const

TOutGeneralspecification specification, UINT vehicleMask)

: ToutProcessor(id, specification).

fVehicleobserver ((TOutObserver*) NULL),

fVehiclestorage(specification.GetRoot (), specification.GetName() + "." + kVehiclesuffix, TOutStorage: :kWrite),

1

fVehicleMask (vehicleMask)

$1 /$ Destroy an event processor.

TOutEventProcessor: : TOutEvent Processor (\})

1

$1 /$ Return the processor type.

TOutProcessor: : EProcessorType TOutEventProcessor: :GetProcessorType() const \{

1

return kEventProcessor;

// Begin recording output for this time step.

void TOutEventProcessor: : Recordoutput (REAL)

\{

\}

return;

// Finish recording output for a vehicle.

void TOutEventProcessor: : RecordVehicle()

1

if (fVehiclestorage.Getoffset () == 0)

fVehicleStorage. WriteHeader (fVehicleobserver->GetRecord ());

\}

fVehiclestorage.Write(fVehicleobserver->GetRecord ());

// Return the vehicle observer.

TOutObserver\& TOutEventProcessor: :GetVehicleobserver()

1

\}

return *fVehicleobserver;

const TOutObserver\& TOutEventProcessor: :GetVehicleobserver( ) const l

return * fvehicleobserver; 
// Define the vehicle observer.

void TOutEventProcessor: : SetVehicleobserver(TOutobserver\& observer)

i

l

fVehicleobserver = sobserver;

// Return the vehicle status mask.

UINT TOutEventProcessor::GetVehicleMask() const

\{

\}

return fVehicleMask;

\section{TOutEventRetriever Class}

\section{EventRetriever.h}

$1 /$ Project: TRANSIMS

// Subsystem: Simulation Output

// \$RCSfile: EventRetriever.h,v $\$$

// SRevision: 0.1 \$

// \$Date: 1996/06/19 17:13:59 \$

// \$State: Stab \$

/1 \$Author: bwb \$

$/ /$ U.S. Government Copyright 1995

$/ /$ All rights reserved

\#ifndef TRANSIMS_OUT_EVENTRETRIEVER "define TRANSIMS_OUT_EVENTRETRIEVER

// Include TRANSIMS header files.

\#include <GBL/Globals.h>

\#include <OUT/Retriever.h>

// An event retriever gets specific event data from storage and

// coordinates its conversion and filtering.

class TOutEventRetriever

I

: public ToutRetriever

public:

// Construct a reader for the specified hosts and given specification,

// and network.

TOutEventRetriever const TOutStorage: :HostSet\& hosts, const

TOutGeneralSpecification specification, const TNetNetwork* network

= NULL):

// Perform the retrieval on the specified writers.

void Retrieve(TOutWriter vehicleWriter, bool sort = TRUE):

private:

// The retriever is connected to a vehicle storage.

l;

TOutStorage fVehiclestorage;

\#endif // TRANSIMS_OUT_EVENTRETRIEVER

\section{EventRetriever.C}

// Project: TRANSIMS

// Subsystem: Simulation Output

// SRCSfile: EventRetriever.C, $\mathrm{s}$

// \$Revision: 0.1 \$

// \$Date: 1996/06/19 17:14:37 \$ 


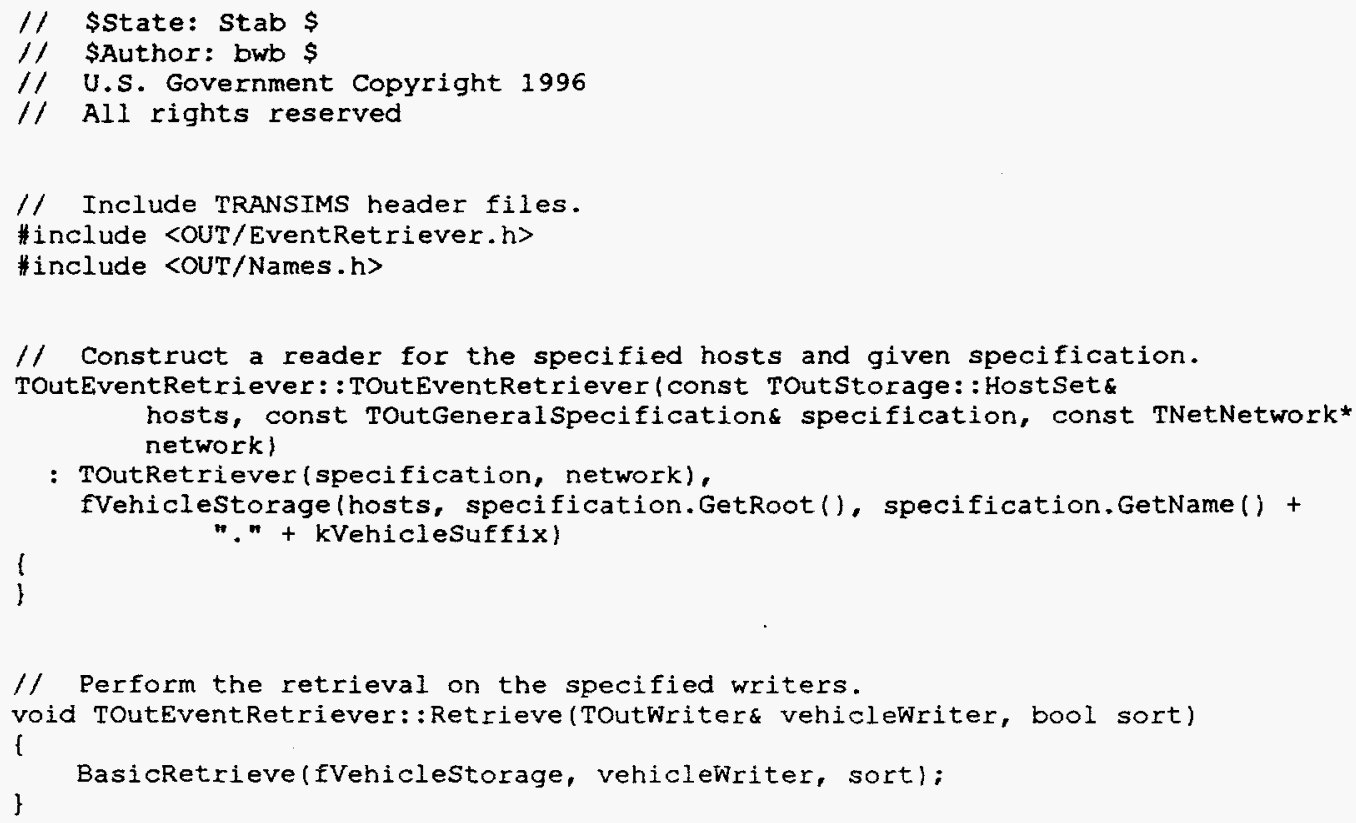

\section{TOutEvolutionProcessor Class}

\section{EvolutionProcessor.h}

$1 /$ Project: TRANSIMS

// Subsystem: Simulation Output

// SRCSfile: EvolutionProcessor.h,v $\$$

/1. SRevision: 0.6 \$

// \$Date: 1996/06/19 17:16:38 \$

$1 /$ SState: Stab \$

$1 /$ \$Author: bwb \$

// U.S. Government Copyright 1995

$/ /$ All right reserved

\#ifndef TRANSIMS OUT EVOLPROC \#define TRANSIMS_OUT_EVOLPROC

// Include TRANSIMS header files.

\#include "OUT/Processor.h"

\#include "OuT/Storage. $h$ "

// Forward declarations.

class TOutEvolutionspecification:

class ToutObserver:

// An output evolution processor deals with evolving data such as

// that needed for animation, waterfall plots, etc.

class ToutEvolutionProcessor

: public ToutProcessor

public:

$1 /$ Construct an evolution processor.

TOutEvolutionProcessor (OutProcessorId, const TOutGeneralspecificationd):

$1 /$ Destruct an evolution processor.

virtual TOutEvolutionProcessor();

// Return the processor type.

EProcessorType GetProcessorType() const; 
$/ /$ Begin recording output for this time step.

virtual void Recordoutput (REAL time):

$/ /$ Finish recording output for a node.

virtual void RecordNode():

// Elnish recording output for a link.

virtual void RecordLink();

// Finish recording output for a vehicle.

virtual vold RecordVehicle();

// Finish recording output for an intersection. virtual void Recordintersection(1):

// Finish recording output for a signal coordinator. virtual void RecordSignalcoordinator (1);

// Finish recording output for a signalized control. virtual void RecordsignalizedControl():

// Return the node observer.

TOutObserver GetNodeObserver ();

const TOutObserver GetNodeObserver() const

1/ Define the node observer.

void SetNodeobserver (TOutObserver\& observer);

// Return the link observer.

TOutObserver\& GetIinkObserver():

const TOutObserver GetLinkObserver() const;

// Define the link observer.

void SetLinkobserver(TOutobserver observer):

// Return the vehicle observer.

TOutObserver \& GetVehicleobserver(1):

const Toutobserver\& GetVehicleobserver() const:

// Define the vehicle observer.

void SetVehicleobserver(TOutObserver\& observer);

// Return the intersection observer.

TOutObserver\& GetIntersectionobserver();

const TOutObserver \& GetIntersectionobserver() const

// Define the intersection observer.

void Set Intersectionobserver(TOutobservers observer):

$1 /$ Return the signal coordinator observer.

TOutObserver\& GetSignalCoordinatorobserver():

const TOutObserver\& GetSignalCoordinatorobserver() const;

$1 /$ Define the signal coordinator observer.

void SetSignalCoordinatorobserver (TOutoosezver\& observer);

// Return the signalized control observer.

TOutObserver GetSignalizedControlobserver();

const TOutobserver \& GetSignalizedControlobserver() const

// Define the signalized control observer.

void SetSignalizedControlobserver(TOutObserver\& observer);

private:

// An evolution processor has a node observer.

TOutObserver* fNodeObserver:

// An evolution processor has a link observer.

TOutObserver* fLinkObserver;

// An evolution processor has a vehicle observer.

TOutObserver* fVehicleobserver;

/f An evolution processor has an intersection observer. 
TOutobserver* fIntersectionobserver;

/f An evolution processor has a signal coordinator observer. TOutObserver* fSignalCoordinatorobserver:

// An evolution processor has a signalized control observer. TOutObserver ${ }^{\text {fSignalizedControlobserver; }}$

// An evolution processor is connected to a vehicle storage. Toutstorage fVehiclestorage;

// An evolution processor is connected to an intersection storage. Toutstorage fIntersectionstorage;

// An evolution processor is connected to a signalized control storage. i: TOutstorage fSignalstorage;

\section{"endif // TRANSIMS_OUT_EVOLPROC}

\section{EvolutionProcessor.C}

$1 /$ Project: TRANSIMS

$/ /$ Subsystem: Simulation Output

// \$RCSfile: EvolutionProcessor.C,v $\$$

// SRevision: 0.6 \$

// \$Date: 1996/06/19 17:15:42 \$

// \$State: Stab \$

// \$Author: bwb \$

// U.S. Government Copyright 1995

// Ali right reserved

// Include TRANSIMS header files.

\#include "OUT/EvolutionProcessor.h"

"include "OUT/Exception.h"

\#include "OUT/Observer.h"

\#include "OUT/Names.h"

$1 /$ Construct an evolution processor.

TOutEvolutionProcessor: :TOutEvolutionProcessorlOutProcessorId id, const TOutGeneralspecifications spec

: ToutProcessor(id, spec),

fNodeobserver ( (TOutObserver*) NULL),

fLinkObserver( (TOutObserver*) NULL),

fVehicleobserver ( (TOutObserver*) NULL),

fIntersectionobserver ( (TOutObserver*) NULL).

fSignalCoordinatorobserver( (TOutObserver*) NULL),

fSignalizedControlobserver ( TOutobserver*) NULL),

fVehicleStorage (spec.GetRoot (), spec.GetName() + " ." + kVehicleSuffix, TOutstorage: : kWrite),

fIntersectionStorage (spec.GetRoot (), spec.GetNamel) + " . + kIntersectionSuffix, ToutStorage: :kWritel,

fSignalstorage (spec.GetRoot (), spec.GetNamel) + "." + kSignalsuffix, TOutStorage: : kWrite)

i

// Destruct an evolution processor.

TOutEvolution Processor: : TOutEvolutionProcessor()

?

// Return the processor type.

TOutProcessor::EProcessorType TOutEvolutionProcessor: :GetProcessorType() const

return kEvolutionProcessor;

\} 


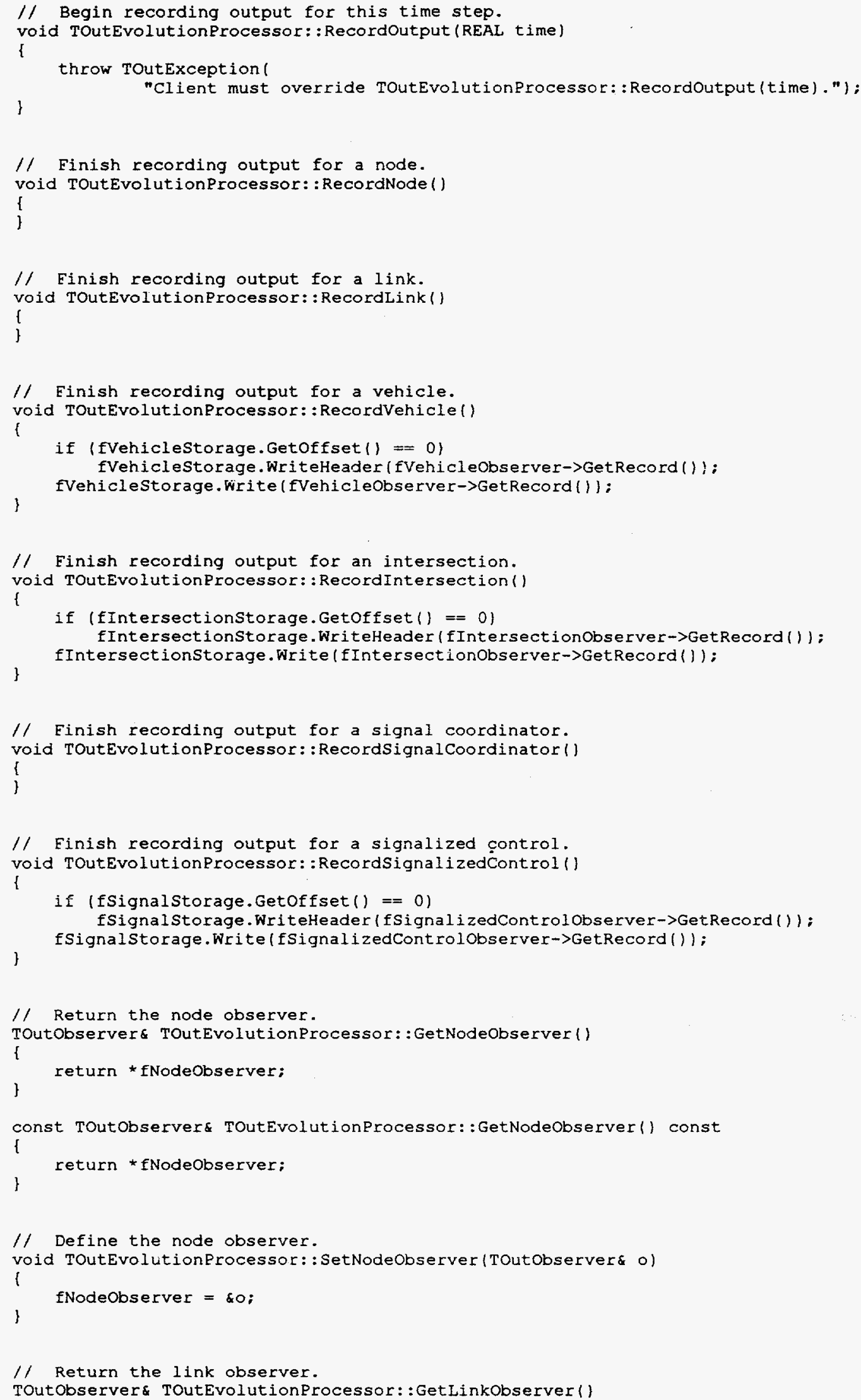


1

return *fLinkObserver:

const TOutObserver TOutEvolutionProcessor: :GetLinkObserver() const

$$
1
$$

return *fLinkobserver;

// Define the link observer.

void TOutEvolutionProcessor: : SetLinkObserver(TOutobserver\& of

\{

\}

fLinkObserver $=80$;

// Return the vehicle observer.

TOutObserver\& TOutEvolutionProcessor: : GetVehicleobserver()

1

)

return *fVehicleobserver;

const TOutObserver TOutEvolutionProcessor: :GetVehicleobserver() const l

1

return *fVehicleobserver;

// Derine the vehicle observer.

void TOutEvolutionProcessor: :SetVehicleObserver(TOutObserver o)

l

)

fVehicleobserver $=\& 0$;

// Return the intersection observer.

TOutObserver TOutEvolutionProcessor: :GetIntersectionobserver()

1

)

return *fIntersectionobserver;

const TOutobserver\& TOutEvolutionProcessor: :GetIntersectionObserver() const l

J

return *Intersectionobserver;

1/ Define the intersection observer.

void ToutEvolutionProcessor: :SetIntersectionobserver(TOutobserver\& of

\{

\}

fIntersectionobserver $=\& 0$;

// Return the signal coordinator observer.

TOutobserver\& TOutEvolutionProcessor::GetSignalCoordinatorobserver ()

i

)

return *fSignalCoordinatorobserver;

const TOutObserver\& TOutEvolutionProcessor: Getsignalcoordinatorobserver() const I

r

return * fSignalCoordinatorobserver;

// Define the signal coordinator observer.

void TOutEvolutionProcessor: SetSignalCoordinatorobserver (TOutobserver o)

1

\}

EsignalCoordinatorobserver $=$ \&

// Return the signalized control observer. 


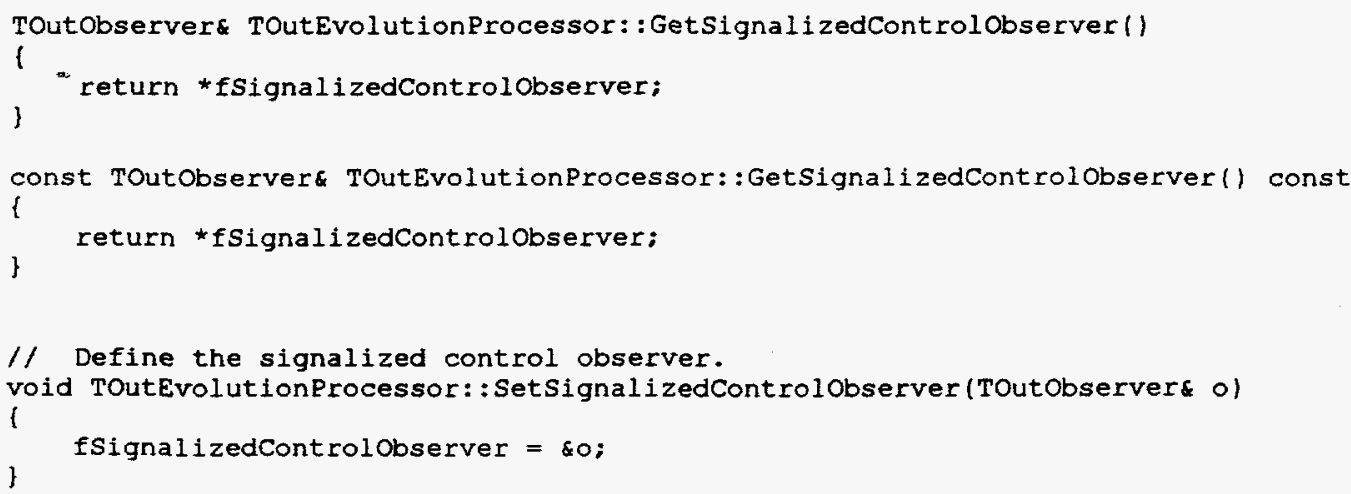

E. TOutEvolutionRetriever Class

\section{EvolutionRetriever.h}

1/ Project: TRANSIMS

// Subsystem: Simulation output

// \$RCSfile: EvolutionRetriever.h,v \$

$/ /$ SRevision: 0.8 \$

$/ /$ \$Date: 1996/06/19 17:21:54\$

$1 /$ \$state: Stab \$

/1 \$Author: bwb \$

// U.S. Government Copyright 1995

// All rights reserved

\#ifndef TRANSIMS OUT EVOLUTIONRETRIEVER \#define TRANSIMS OUT EVOLUTIONRETRIEVER

// Include TRANSIMS header files.

\#include <GBL/Globals.h>

\#include <out/Retriever.h>

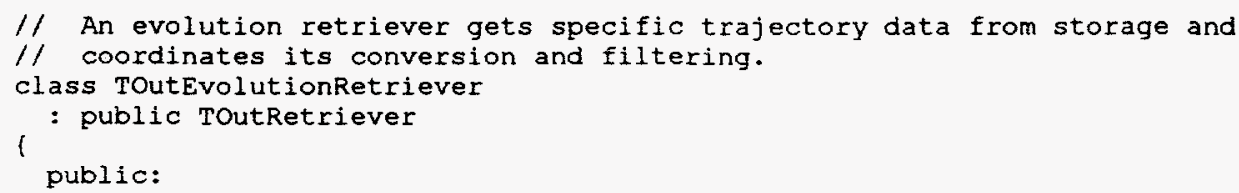

$/ /$ Construct a reader for the specified hosts and given specification, $/ /$ and network.

TOutEvolutionRetriever(const TOutStorage: HostSet $\&$ hosts, const TOutGeneralSpecification s specification, const TNetNetwork* network $=$ NULL):

// Perform the retrieval on the specified writers.

void Retrieve(TOutWriter vehiclewriter, Toutwriter\& intersectionwriter, TOutWriter signalWriter, bool sort = TRUEl;

private:

// The retriever is connected to a vehicle storage.

Toutstorage fVehiclestorage;

1/ The retriever is connected to an intersection storage.

ToutStorage fIntersectionstorage:

$1 /$ The retriever is connected to a signal storage.

l; Toutstorage fsignalstorage;

\#endif // TRANSIMS_OUT_EVOLUTIONRETRIEVER 


\section{EvolutionRetriever.C}

$1 /$ Project: TRANSIMS

// Subsystem: Simulation Output

// SRCSfile: EvolutionRetriever.C,v \$

$1 /$ SRevision: 0.9 \$

// SDate: 1996/06/19 17:21:35 \$

// \$state: Stab \$

$1 /$ SAuthor: bwb \$

// U.S. Government Copyright 1995

$/ /$ All rights reserved

// Include TRANSIMS header files.

\#include <OUT/EvolutionRetriever.h>

\#include <OUT/Names.h>

// Construct a reader for the specified hosts and given specification. TOutEvolutionRetriever: :TOutEvolutionRetriever(const TOutStorage: : HostSet\&

hosts, const ToutGeneralspecifications specification, const TNetNetwork* network)

: ToutRetriever (specification, network),

fVehicleStorage(hosts, specification. GetRoot(1), specification.GetName() + "." + kVehiclesuffix?,

fIntersectionstorage (hosts, specification.GetRoot(), specification.GetName() $+" . "+$ kIntersectionsuf $f(x)$.

fSignalstorage (hosts, specification.GetRoot(), specification.GetName() + 1 "." + kSignalsuffix)

// Perform the retrieval on the specified writers.

void TOutEvolutionRetriever: : Retrieve(TOutWriter\& vehiclewriter,

1

ToutWriter intersectionWriter, ToutWriter\& signalWriter, bool sort)

BasicRetrieve(fVehiclestorage, vehiclewriter, sort);

BasicRetrieve (fIntersectionstorage, intersectionWriter, sort):

子

BasicRetrieve(fSignalstorage, signalWriter, sort);

\section{F. TOutException Class}

\section{Exception.h}

$1 /$ Project: TRANSIMS

// Subsystem: Simulation output

$/ /$ SRCSfile: Exception.h, $\mathrm{v}$ \$

// SRevision: 0.6 \$

// \$Date: 1996/06/19 17:22:59\$

$1 /$ \$State: Stab $\$$

1/ \$Author: bwb \$

// U.S. Government Copyright 1995

// All rights reserved

\#ifndef TRANSIMS_OUT_EXCEPTION \#define TRANSIMS_OUT_EXCEPTION

// Include TRANSIMS header files. \#include <GBL/Globals.h>

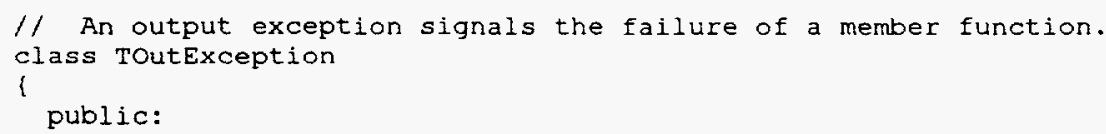


// Construct a copy of the specified exception.

// TOutException(const ToutExceptions exception);

1/ Make the exception a copy of the specified exception.

// TOutExceptions operator=(const TOutExceptions exception);

// Return the message text for the exception.

const string GetMessagel/ const;

private:

// Each exception has a message.

\} ; string fMessage;

// This exception is thrown when a storage operation fails. class ToutStorageFailure

1

: public TOutException

public:

// Construct an exception with the specified message text. TOutStorageFailure(const string\& message = "Storage failure.");

// Construct a copy of the specified exception.

// TOutStorageFailure(const TOutStorageFailure\& exception);

// Make the exception a copy of the specified exception.

// TOutStoragefailure\& operator=(const TOutStorageFailure\& exception);

; ;

// This exception is thrown when a writer operation fails.

class TOutWriterFailure

\{

: public TOutException

public:

$1 /$ Construct an exception with the specified message text.

TOutWriterFailure(const string\& message = "Writer failure.");

// Construct a copy of the specified exception.

// TOutWriterFailure(const TOutWriterFailure exception);

// Make the exception a copy of the specified exception.

// TOutWriterFailure\& operator=(const TOutWriterFailure\& exception);

:

$1 /$ This exception is thrown when the processor type is invalid.

class TOutInvalidProcessor

\{

: public ToutException

public:

// Construct an exception with the specified message text.

TOutInvalidProcessor(const strings message = "Invalid Processor.");

// Construct a copy of the specified exception.

// ToutInvalidProcessor(const ToutInvalidProcessor \& exception):

// Make the exception a copy of the specified exception.

// TOutInvalidProcessor\& operator=(const TOutInvalidProcessor\& exception):

; ;

\# endif // TRANSIMS_OUT_EXCEPTION

2. Exception.C

// Project: TRANSIMS

// Subsystem: Simulation Output

// \$RCSfile: Exception.c, $v \$$ 


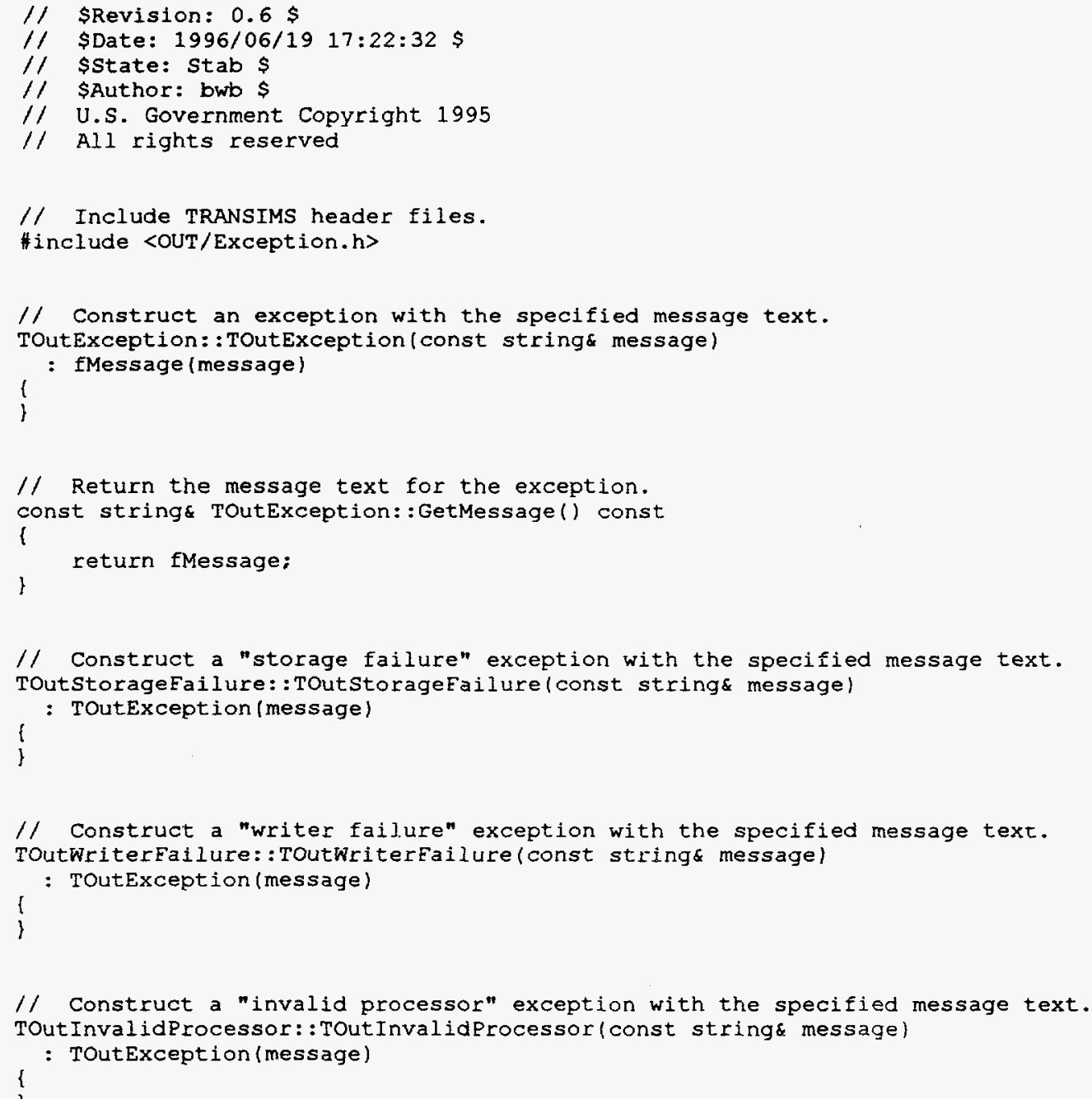

\section{G. TOutFactory Class}

\section{Factory.h}

$/ /$ Project: TRANSIMS

// Subsystem: Simulation Output

$1 /$ \$RCSfile: Factory.h, v \$

// SRevision: 0.4 \$

// \$Date: 1996/06/19 17:23:26\$

// SState: Stab \$

1/ \$Author: bwb \$

// U.S. Government Copyright 1995

// All right reserved

\#i fndef TRANSIMS OUT FACTORY \#define TRANSIMS_OUT_FACTORY

// Include TRANSIMS header files. \#include "OUT/Id.h"

$1 /$ Forward declarations.

class TOutEvolutionProcessor;

class TOutEventProcessor;

class ToutSummaryProcessor: 


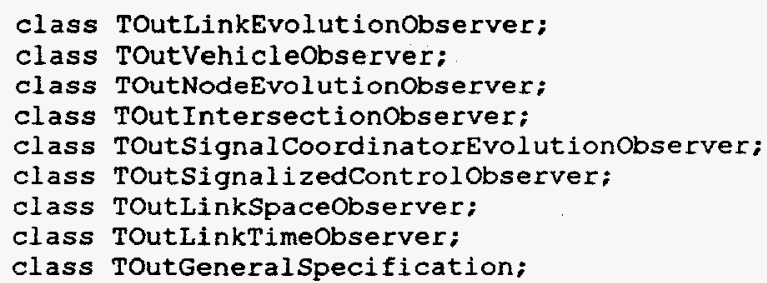

// An output factory allocates and constructs new output objects. class Toutfactory

I

public:

$1 /$ Construct a factory. ToutFactory ():

// Destruct a factory. virtual TOutFactory();

/f Return a new evolution processor from the specification.

virtual TOutEvolutionProcessor* NewEvolutionProcessor OutProcessorId id, const ToutGeneralspecifications specification $>0$;

// Return a new event processor from the specification.

virtual TOutEventProcessor* NewEventProcessorloutProcessorId id, const ToutGeneralspecification specification $=0$;

// Return a new summary processor from the specification.

virtual TOutSummaryProcessor* NewSummaryProcessor(OutProcessorId id, const TOutGeneralspecification specification) $=0$;

// Return a new link evolution observer.

virtual TOutLinkEvolutionobserver* NewLinkEvolutionobserver lOutobserverId id) $=0$;

// Return a new vehicle observer.

virtual TOutVehicleObserver NewVehicleobserver(OutObserverId id) = 0 ;

// Return a new node evolution observer.

virtual TOutNodeEvolutionObserver* NewNodeEvolutionObserver (OutObserverId id) $=0$;

// Return a new intersection observer.

virtual TOutIntersectionObserver* NewIntersectionobserver (OutobserverId id) $=0$

// Return a new signal coordinator evolution observer.

virtual TOutSignalCoordinatorEvolutionobserver* NewSignalCoordinatorEvolutionobserver (OutobserverId id) $=0$;

// Return a new signalized control observer.

virtual TOuisignatizedControlobserver* NewSignalizedControlobserver (OutObserverId id) $=0$;

// Return a new link space observer.

virtual TOutLinkSpaceObserver* NewLinkSpaceObserver(OutObserverId id) $=0$;

1/ Return a new link time observer.

virtual TOutLinkTimeObserver* NewLinkTimeObserver(OutobserverId id) =0:

\};

\#endif $/ /$ TRANSIMS_OUT EACTORY

\section{Factory.C}

$1 /$ Project: TRANSIMS

// Subsystem: Simulation Output

// SRCsfile: Eactory.C,v $\$$

// SRevision: $0.3 \mathrm{~s}$

// SDate: 1996/06/19 17:23:15\$ 


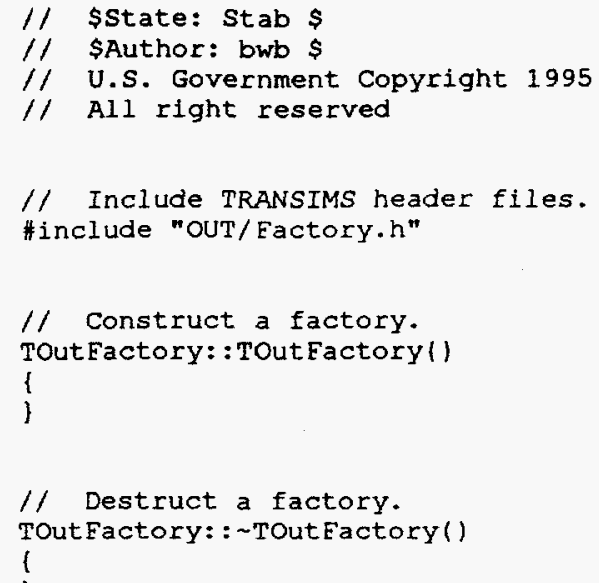

\section{H. TOutGeneralSpecification Class}

\section{GeneralSpecification.h}

$1 /$ Project: TRANSIMS

// Subsystem: Simulation Output

// \$RCSfile: Generalspecification.h,v \$

// SRevision: $0.6 \$$

// \$Date: 1996/06/19 17:24:30\$

$1 /$ \$State: Stab \$

$1 /$ \$Author: bwb \$

// U.S. Government Copyright 1995.

// All rights reserved

\#ifndef TRANSIMS OUT GENERALSPECIFICATION \#define TRANSIMS_OUT_GENERALSPECIFICATION

// Include Booch Components header files. \#include <BCstorem.h>

$\#$ include $\langle$ BCSetU.h>

// Include TRANSIMS header files.

\#include <GBL/Globals.h>

\#include 〈NET/Id.h>

\#include <NET/Rectangle.h〉

\#include <OUT/GeneralspecificationReader.h>

1/ The general specification defines the frequency and extent of data to be

$/ /$ collected or retrieved in both space and time.

class TOutGeneralspecification

i

public:

$1 /$ Type definitions.

typedef BC TUnboundedSet<NetNodeId, 10000U, BC CManaged> NodeIdSet;

typedef $B C$-TSetActiveIterator<NetNodeId> NodeI'setIterator;

typedef BC-TUnboundedSet<NetLinkId, 10000 , BC CManaged> LinkIdSet;

typedef BC_TSetActiveIterator<NetLinkId> LinkId̄SetIterator;

1/ Time constants.

static const REAL kMinusinfinity;

static const REAL kPlusInfinity;

// Construct a general specification from a reader.

TOutGeneralSpecification (TOutGeneralSpecificationReader \& reader);

// Return the root for the specification.

const string\& GetRoot (1) const: 
// Return the name for the specification. const string\& GetName() const;

// Return whether data should be collected for the specified time. bool CollectForTime(REAL time) const;

// Return whether data should be sampled at the specified time. bool SampleForTime(REAL time) const;

// Return whether the specified time is the start time. bool IsAtStartTime(REAL time) const;

// Return the box length. REAL GetBoxLength() const;

// Return whether data should be collected for the specified point in // space.

bool CollectForPoint (const TGeoPoint\& point) const;

// Return whether data should be collected for the specified node. bool CollectForNode (NetNodeId id) const;

// Return whether data should be collected for the specified link. bool CollectForlink (NetLinkId id) const;

private:

// Each specification has a root.

string fRoot;

// Each specification has a name.

string fName;

// Each specification has a minumum time.

REAL fTimeMinimum;

// Each specification has a maximum time.

REAL fTimeMaximum:

// Each specification has a time step.

REAL fTImeStep;

// Each specification has a time sample.

REAL fTimeSample;

// Each specification has a box length.

REAL fBoxLength;

// Each specification has a collection region.

TGeoRectangle fRegion;

// Each specification has a set of node ids. NodeIdSet fNodes:

// Each specification has a set of link ids. LinkIdSet flinks;

\}

\#endif // TRANSIMS_OUT_GENERALSPECIFICATION

\section{GeneralSpecification.C}

// Project: TRANSIMS

// Subsystem: simulation Output

// \$RCSfile: Generalspecification.C, $\mathrm{s}$

// SRevision: 0.7 \$

1/ \$Date: 1996/06/19 17:24:13 \$

$1 /$ \$state: Stab $\$$

// \$Author: bwb \$

$/ /$ U.S. Government Copyright 1995

// All rights reserved 
$1 /$ Include TRANSIMS header files.

\#include <OUT/Generalspecification.h>

$1 /$ Define the hash function for node ids.

static BC_Index NodeIdHashValue(const NetNodeId\& id)

1

1

return BC_Index (id):

// Define the hash function for link ids. static BC_Index LinkIdHashValue(const NetLinkId\& id) I

1

return BC_Index(id):

$1 /$ Time constants.

const REAL TOutGeneralSpecification: :kMinusinfinity = (UINT) -2;

const REAL TOutGeneralspecification::kPlusInfinity $=$ (UINT) -1 ;

// Construct a general specification from a reader.

TOutGeneralSpecification: :TOutGeneralSpecification( ToutGeneralspecificationReader\& reader)

: fRoot (reader.GetRoot (1)),

fName (reader. GetName (1),

fTimeMinimum (reader.GetMinimumTime()),

fTimeMaximum (reader.GetMaximumTime ()),

fTimeStep (reader.GetTimeStep (i),

fTimesample (reader.GetTimesamplé()).

fBoxLength (reader. GetBoxLength ()),

fRegion (reader.GetRegion()),

fNodes (reader. GetNodes ()),

\{

fLinks (reader.GetIinks ())

// Return the root for the specification.

const string\& TOutGeneralSpecification: :GetRoot() const

i

1

return fRoot;

// Return the name for the specification.

const string\& TOutGeneralSpecification: GetName() const

1

return fName;

)

// Return whether data should be collected for the specified time. bool TOutGeneralspecification: : CollectForTime (REAL time) const

\{

if (fTimeMinimum != kMinusInfinity \& time < fTimeMinimum) return FALSE;

if (fTimeMaximum $:=$ kPlusinfinity \&\& time > fTimeMaximum) return FALSE;

const REAL start = fTimeMinimum $!=$ kMinusInfinity $?$ fTimeMinimum : 0 ;

)

return int $(100 *($ time - start $))$ int $(100 *$ fTimestep $)==0$;

// Return whether data should be sampled at the specified time.

bool TOutGeneralSpecification: SampleEorTime(REAL time) const

\{

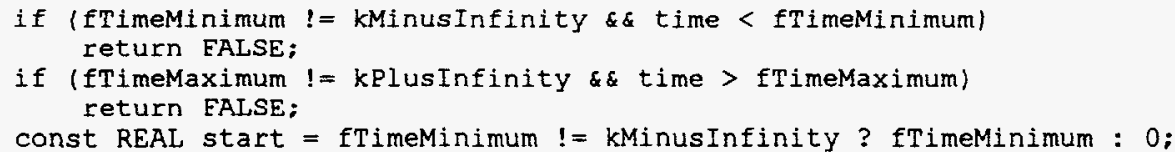


return int $(100 *($ time - start $))$ int $(100 *$ fTimeSample $)=0$;

// Return whether the specified time is the start time.

bool TOutGeneralspecification: IsAtStartTime(REAL time) const

\{

\}

return fTimeMinimum = time;

// Return the box length.

REAL TOutGeneralspecification: :GetBoxLength() const

i

\}

return fBoxLength;

// Return whether data should be collected for the specified point in space. bool TOutGeneralSpecification:; CollectForPoint(const TGeoPoint\& point) const

l

\}

return fRegion. Contains (point);

// Return whether data should be collected for the specified node.

bool TOutGeneralspecification: CollectForNode (NetNodeId id) const

I

了

return fNodes. IsMember(id);

// Return whether data should be collected for the specified link.

bool TOutGeneralspecification: CollectForLink(NetLinkId id) const

i

return flinks. IsMember(id);

\}

\section{TOutGenera/SpecificationReader Class}

\section{GeneralSpecificationReader.h}

$1 /$ Project: TRANSIMS

// Subsystem: Simulation Output

// \$RCSfile: GeneralspecificationReader.h, $v$ \$

// SRevision: $0.6 \$$

// \$Date: 1996/06/19 17:26:00\$

// \$State: Stab \$

// SRuthor: bwb \$

// U.S. Government Copyright 1995

// All rights reserved

\#i fndef TRANSIMS_OUT_GENERALSPECIFICATIONREADER \#define TRANSIMS_OUT_GENERALSPECIFICATIONREADER

$1 /$ Include Booch Components header files.

$\#$ include <BCStoreM.h>

$\#$ include $\langle B C S e t U . h\rangle$

$1 /$ Include TRANSIMS header files.

\#include <GBL/Globals.h>

\#include <DBS/Accessor.h>

\#include <NET/Id.h>

\#include <NET/Rectangle.h>

$1 /$ Forward declarations.

class ToutSpecificationReader; 
// A general specification reader reads specifications from the database. class ToutGeneralSpecificationReader

1

public:

// Type definitions.

typedef BC_TUnboundedSet<NetNodeId, 10000U, BC_CManaged> NodeIdSet;

typedef $B C$ TSetActiveIterator $<$ NetNodeId> NodeI dsetIterator;

typedef $\mathrm{BC}^{-}$TUnboundedSet<NetIinkId, 10000U, BC CManaged> LinkIdSet;

typedef BC_TSetActiveIterator<NetLinkId> LinkIdSetIterator:

// Processor types.

enum EProcessorType (kEvolutionProcessor, kEventProcessor, kSummaryProcessor):

$1 /$ Construct a general specification reader.

TOutGeneralSpecificationReader(TOutSpecificationReader reader);

// Construct a copy of the given general specification reader.

// TNetGeneralSpecificationReader(const TNetGeneralSpecificationReader\&

// reader);

// Make the reader a copy of the given general specification reader.

// TNetGeneralspecificationReader $\&$ operator $=$ (const

// TNetGeneralSpecificationReader \& reader);

$1 /$ Reset the iteration over the table.

void Reset (1);

$1 /$ Get the next specification in the table.

void GetNextspecification():

// Return whether there are any more specifications in the table.

bool Morespecifications() const:

// Return the root of the specification.

string GetRoot() const;

// Return the name of the specification.

string GetName() const;

// Return the minimum time of the specification.

REAL GetMinimumTime() const;

// Return the maximum time of the specification.

REAL GetMaximumime() const;

// Return the time step of the specification.

REAL GetTimestep \{ const:

// Return the time sampling of the specification.

REAL GetTimeSample() const;

// Return the box length of the specification.

REAL GetBoxI ...yih!; const;

// Return the geographic region of the specification.

TGeoRectangle GetRegion() const;

// Return the nodes in the specification.

NodeIdSet GetNodes() const;

// Return the links in the specificaiton.

LinkIdSet GetLinks() const;

$1 /$ Return the processor type in the specification. A TOutInvalidProcessor

// exception is thrown if the processor is not a valid type.

EProcessorType GetProcessorType() const:

private:

// Each general specification reader has a database table accessor.

TDbAccessor fAccessor;

// Each general specification reader accesses a node table. 
TDbAccessor fNodeAccessor;

// Each general specification reader accesses a link table. TDbAccessor fLinkAccessor;

// Each general specification has a processor type. const TDbField fProcessorfield;

// Each general specification has a root. const TDbField fRootField;

// Each general specification has a name. const TDbField fNameField;

// Each general specification has a minimum time. const TDbEield fMinimumTimeField:

// Each general specification has a maximum time. const TDbField fMaximumTimeField;

// Each general specification has a time step. const TDbField fTimestepField;

// Each general specification has a time sample. const TDbField fTimesamplefield;

// Each general specification has a box length. const TDbField fBoxLengthField;

// Each general specification has a minimum abscissa. const TDbField fMinimumabscissafield;

// Each general specification has a maximum abscissa. const TDbField fMaximumabscissafield:

// Each general specification has a minimum ordinate. const TDbField fMinimumordinateField

// Each general specification has a maximum ordinate. \} : const TDbField fMaximumordinateField

\#endif // TRANSIMS_OUT_GENERALSPECI FICATIONREADER

\section{GeneralSpecificationReader.C}

// Project: TRANSIMS

// Subsystem: Simulation Output

// SRCSfile: GeneralspecificationReader.C, $v \$$

1/ SRevision: 0.6 \$

// \$Date: 1996/06/19 17:25:26 \$

// \$state: Stab \$

11 SAuthor: bwb $\$$

$1 /$ U.S. Government Copyright 1995

// All rights reserved

// Include TRANSIMS header files.

\#include <ouT/Exception.h>

\#include <OUT/SpecificationReader.h>

\#include <OUT/GeneralspecificationReader.h>

// Define the hash function for node ids. static BC_Index NodeIdHashValue(const NetNodeId\& id) l

,

$$
\text { return BC_Index(id); }
$$

1/ Define the hash function for link ids. static BC Index LinkIdHashValue (const NetLinkId\& id) 1 
// Construct a general specification reader.

TOutGeneralSpecificationReader: : TOutGeneralSpecificationReader TOutSpecificationReader\& reader)

: fAccessor (reader.GetGeneralTable()), fNodeAccessor (reader.GetNodeTable()), fLinkAccessor (reader.GetLinkTable()), fProcessorField (reader. GetGeneralTable (). GetField("PROCESSOR")), fRootField (reader. GetGeneralTable (). Get Eield ("ROOT")), fNameField (reader. GetGeneralTable (). GetEield ("NAME")], fMinimumTimeField (reader.GetGeneralTable ().GetField("TIMEMIN"),, fMaximumTimeField (reader.GetGeneralTable().GetField ("TIMEMAX")), fTimeStepField (reader.GetGeneralTable().GetField ("TIMESTP")), fTimeSampleField (reader. GetGeneralTable (). GetField("TIMESMP")), fBoxLengthField (reader.GetGeneralTable (). GetField("BoXLEN")), fMinimumAbscissa Field (reader.GetGeneral Table ().GetField ("ABSCISSAMN")), fMaximumAbscissaField (reader.GetGeneral Table (). GetField ("ABSCISSAMX")), fMinimumordinateField (reader.GetGeneral Table (). GetField ("ORDINATEMN")), fMaximumordinateField (reader.GetGeneralTable().GetField("ORDINATEMX"))

// Reset the iteration over the table. void TOutGeneralspecificationReader: : Reset () 1 \}

fAccessor. GotoFirst():

// Get the next specification in the table. void TOutGeneralspecificationReader: :GetNextSpecification() l

\}

faccessor. GotoNext ();

1/ Return whether there are any more specifications in the table. bool TOutGeneralSpecificationReader: MoreSpecifications() const i \}

return fAccessor. IsAtRecord();

1/ Return the root of the specification.

string ToutGeneralspecificationReader: GetRoot() const

l

string root;

fAccessor. GetField(fRootField, root):

) return root;

// Return the name of the specification. string TOutGeneralspecificationReader: :GetName() const

\{

string name;

fAccessor. GetField (fNameField, name);

\}

return name;

// Return the minimum time of the specification.

REAL TOutGeneralSpecificationReader: :GetMinimumTime() const

1

REAL time;

fAccessor.GetField(fMinimumTimeField, time);

\} return time;

// Return the maximum time of the specification. 
REAL TOutGeneralSpecificationReader::GetMaximumTime() const

1

REAL time;

fAccessor.GetEield(fMaximumTimeField, time): return time:

1/ Return the time step of the specification.

REAL TOutGeneralspecificationReader::GetTimestep() const

REAI time

fAccessor.GetField(fTimestepField, time):

return time:

// Return the time sampling of the specification.

REAL TOutGeneralSpecificationReader::GetTimeSample | const

I

REAL time:

fAccessor.GetEield (fTimeSampleField, time) :

return time;

\}

1/ Return the box length of the specification.

REAL TOutGeneralSpecificationReader: :GetBoxLength() const

\{

REAI length;

fAccessor.GetField(fBoxLengthField, length):

return length;

)

// Return the geographic region of the specification.

TGeoRectangle TOutGeneralSpecificationReader: :GetRegion() const

REAL $x 0, x 1, y 0, y^{1}$;

fAccessor.GetField (fMinimumAbscissaField, $x 0$ ):

fAccessor. Get Field (fMaximumAbscissaField, x1):

fAccessor. GetField (fMinimumordinateEield, y0):

fAccessor.GetField (fMaximumordinateField, y1):

return TGeoRectangle (TGeoPoint $\left(x_{0}\right.$, y0), TGeoPoint $\left.\left(x_{1}, y_{1}\right)\right)$;

)

// Return the nodes in the specification.

TOutGeneralspecificationReader: : NodeIdSet

TOutGeneralspecificationReader:: GetNodes() const

NodeIdSet nodes (NodeIdHashvalue):

string current;

fAccessor. GetField (fNameField, current :

const TDbField nameField("NAME"):

const TDbField nodeField ("NODE"):

for ((TDbAccessor \&) fNodeAccessor).GotoFirst () : ((TDbAccessor\&)

fNodeAccessor). IsAtRecord (); ((TDbAccessor $\alpha$ )

fNodeAccessor). GotoNext ()) (

string name:

fNodeAccessor. GetField (nameField, name) :

if (name $==$ current) (

NetNodeId id:

fNodeAccessor.GetField (nodeField, id); nodes. Add (id)

)

return nodes

// Return the links in the specification. 


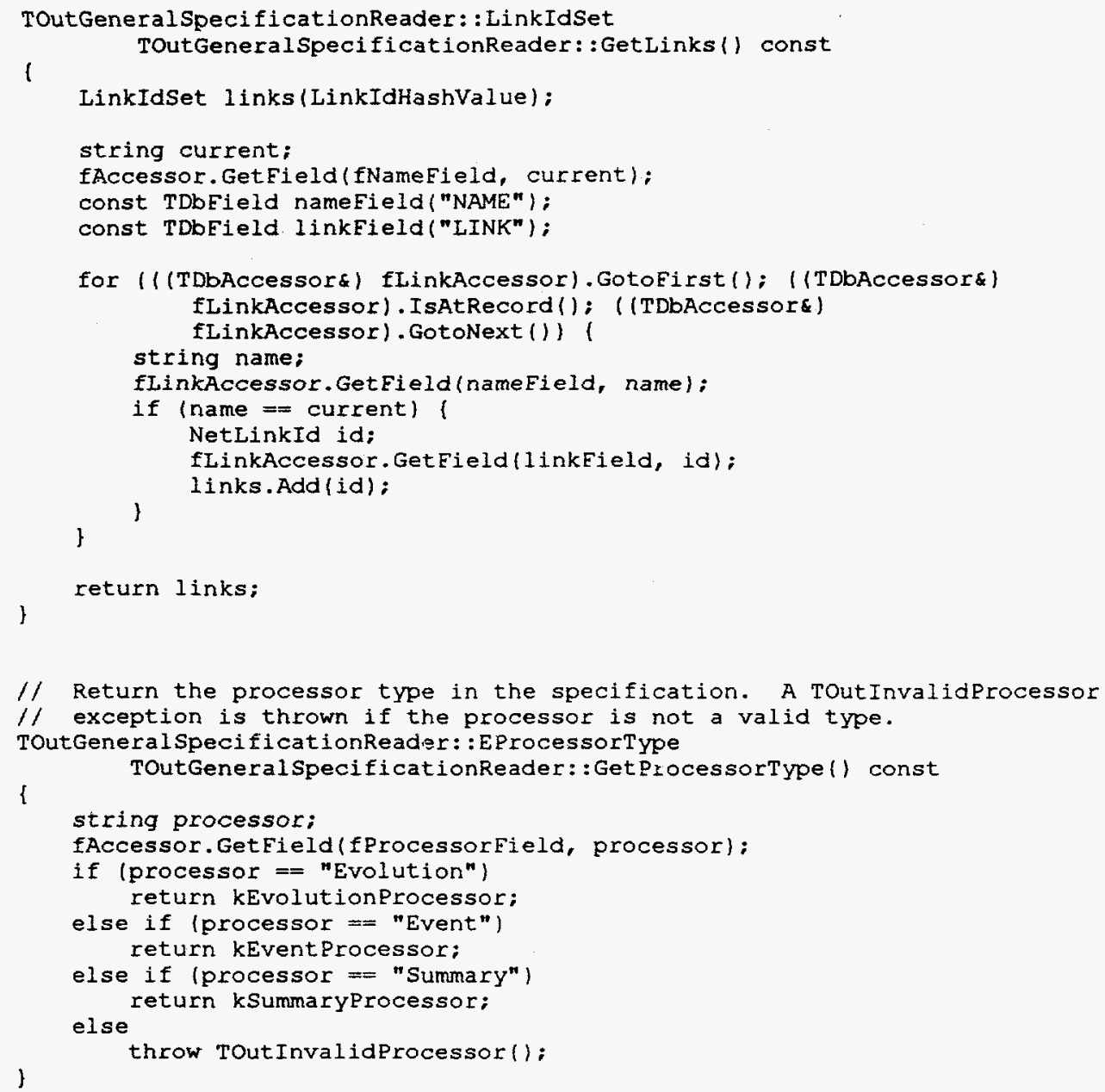

\section{J. TOut/ntersectionObserver Class}

\section{IntersectionObserver.h}

$1 /$ Project: TRANSIMS

// Subsystem: Simulation Output

// \$RCSfile: Intersectionobserver.h, $v \$$

// SRevision: $0.2 \$$

// \$Date: 1996/06/19 17:27:07 \$

// SState: Stab $\$$

// \$Author: bwb \$

$1 /$ U.S. Government Copyright 1995

// All right reserved

\#ifndef TRANSIMS OUT INTOBS

\#define TRANSIMS_OUT_INTOBS

$/ /$ Include TRANSIMS header files.

\#include "OUT/Observer.h"

\#include "NET/Id.h"

// A intersection observer observes data related to intersections.

class Tout Intersectionobserver

: public TOutObserver 1

public: 
$1 /$ Construct an intersection observer

TOutIntersectionobserver (OutobserverId id):

// Destruct an intersection observer.

virtual TOutIntersectionobserver();

protected:

// Define values for the fields being observed.

// The Observe method will use these methods.

// Define the vehicle's id.

vold setId(UINT id);

$1 /$ Define the id of the link the vehicle entered from.

void SetLink (NetLinkId link);

$/ /$ Define the lane number the vehicle entered from.

void SetLane (NetLaneNumber lane);

1/ Define the id of the node the intersection is associated with. void SetNode(NetNodeId node);

1/ Define the index of the vehicle's position in the queue. void setQIndex (BYTE index);

;

\#endif // TRANSIMS_OUT_INTOBS

\section{IntersectionObserver.C}

$1 /$ Project: TRANSIMS

// Subsystem: Simulation Output

// \$RCSfile: Intersectionobserver.C, $\mathrm{s}$

$1 /$ \$Revision: $0.2 \$$

// \$Date: 1996/06/19 17:26:43 \$

$/ /$ \$State: Stab \$

// SAuthor: bwb $\$$

// U.S. Government Copyright 1995

$/ /$ All right reserved

// Include TRANSIMS header files.

\#include "OUT/Intersectionobserver.h"

\#include "OUT/Record.h"

\#include "OuT/Names. $h$ "

$1 /$ Construct an intersection observer

TOut Intersectionobserver: : TOut Intersectionobserver (OutobserverId id)

: TOutobserver(id)

i

SetTime $(-99$.$) ;$

SetId $(-99)$ :

SetLink (-99);

SetLane $(-99)$;

SetNode (-99):

)

SetQIndex $(-99)$;

// Destruct an intersection observer.

TOutIntersectionObserver: : TOut IntersectionObserver()

i

// Define the vehicle's id.

void ToutIntersectionobserver: :SetId(UINT id)

l

)

GetRecord(). SetField(kVehiclefield, id); 


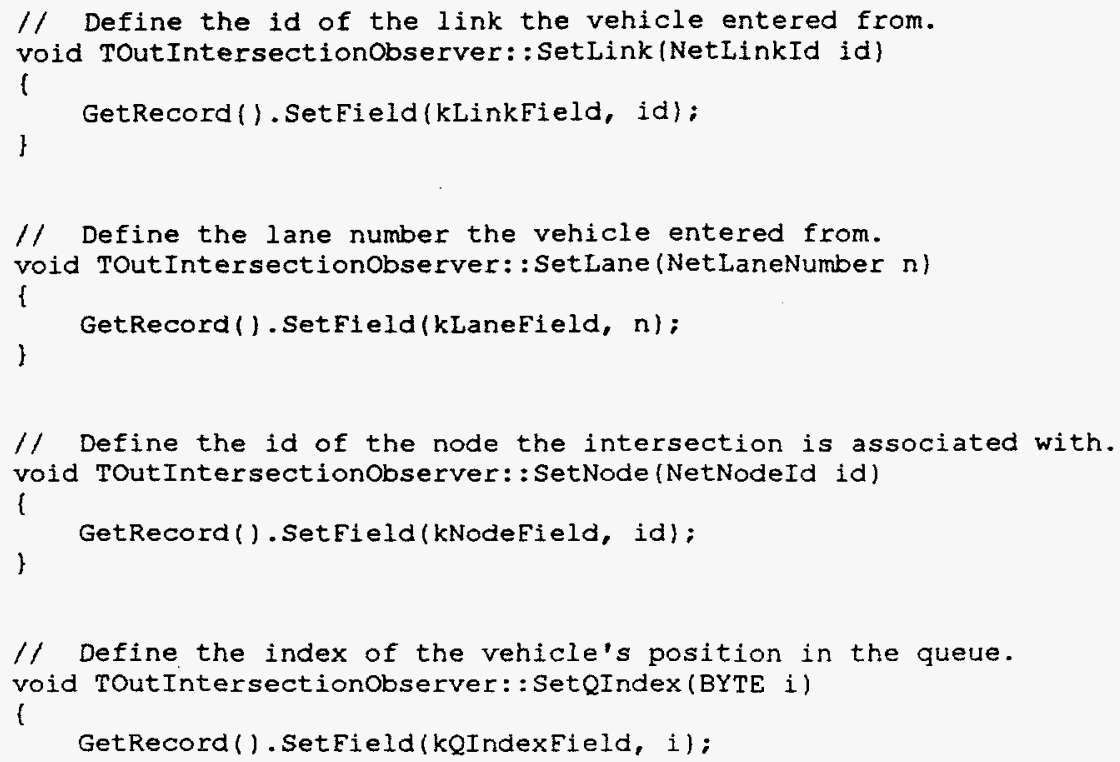

\section{K. TOutLinkEvolutionObserver Class}

1. TOutLinkEvolutionObserver.h

$1 /$ Project: TRANSIMS

// Subsystem: Simulation Output

// SRCSfile: LinkEvolutionobserver.h,v $\$$

// \$Revision: 0.4 \$

// \$Date: 1996/06/19 17:28:06\$

/f SState: Stab \$

// SAuthor: bwb \$

// U.S. Government Copyright 1995

// All right reserved

\#ifndef TRANSIMS OUT LINKEVOLOBS

\#define TRANSIMS_OUT_LINKEVOLOBS

// Include TRANSIMS header files.

\#include "OUT/Observer.h"

// A link evolution observer observes evolving data related to links.

class TOutLinkEvolutionobserver

: public Toutobserver

public:

// Construct a link evolution observer.

ToutlinkEvolutionobserver(OutobserverId id);

// Destruct a link evolution observer.

\}

virtual w TOutLinkEvolutionobserver()

\#endif // TRANSIMS OUT LINKEVOLOBS

2. TOutLinkEvolutionObserver.C

/f Project: TRANSIMS

// Subsystem: Simulation Output

// \$RCSfile: LinkEvolutionobserver.C, $\mathrm{S}$ 
$1 /$ SRevision: 0.4 \$

l/ \$Date: 1996/06/19 17:27:49\$

11 SState: Stab $\$$

// \$Author: bwb \$

$/ /$ U.S. Government Copyright 1995

// All right reserved

$/ /$ Include TRANSIMS header files.

\#include "OUT/LinkEvolutionobserver.h"

// Construct a link evolution observer.

TOutLinkEvolutionobserver: : TOutLinkEvolutionobserver (OutObserverId id)

\{

: TOutobserver(id)

// Destruct a link evolution observer.

TOutLinkEvolutionobserver: : TOutLinkEvolutionobserver()

1

\section{TOutLinkSpaceObserver Class}

\section{LinkSpaceObserver.h}

$1 /$ Project: TRANSIMS

// Subsystem: Simulation Output

// \$RCSfile: LinkSpaceObserver.h, $\mathrm{v} \$$

// SRevision: 0.1 \$

// SDate: 1996/06/19 17:29:18 \$

// \$State: Stab \$

$1 /$ \$Author: bwb \$

// U.S. Government Copyright 1995

// All right reserved

\#ifndef TRANSIMS OUT LINKSPACEOBSERVER \#define TRANSIMS_OUT_LINKSPACEOBSERVER

// Include Booch Components header files.

\#include <BCStoreM.h>

\#include <BCCollu.h>

// Include TRANSIMS header files.

\#include "OUT/Observer.h"

\#include "NET/Id.h"

// A link space observer summarizes vehicle spatial data on a link.

class TOutLinkSpaceObserver

: public Toutobserver

public:

// Construct a link space observer.

TOutLinkSpaceobserver (OutObserverId id):

// Destroy a link space observer.

virtual TOutLinkspaceobserver (1)

protected:

// Return whether the observer has been initialized.

bool IsInitialized() const;

// Clear the box data for the observer.

void ClearBoxData (); 
$1 /$ Set the link id.

void SetLink(NetLinkId id):

$1 /$ set the departure node id.

void SetNode (NetNodeId id);

// Set the link, box, and cell lengths.

void SetLengths(REAL i inkLength, REAL boxLength, REAL cellilength = 0 );

// Add a vehicle.

void AddVehicle(REAL distance, REAL velocity):

// Report the observations to the specified processor.

void Reportobservations (TOutProcessor \& processor);

private:

// A box datum stores the tally for a box.

class TBoxDatum

1

public:

$1 /$ Construct a box datum.

TBoxDatum ()

: fCount $(0)$

1

fVelocityTotal(0)

1

// Make the box datum a copy of the specified box datum.

//TBoxDatum(const TBoxDatums boxDatum):

$1 /$ Assign a box datum.

//TBoxDatum\& operator=(const TBoxDatum\& boxDatum):

// Test equality of box datums.

bool operator $= \pm$ (const TBoxDatum\& boxDatum) const

I

,

return this $==$ sboxDatum;

$1 /$ Test inequality of box datums.

bool operator!=(const TBoxDatum\& boxDatum) const

1

\}

return this != sboxDatum;

// Clear the data in a box.

void Clear()

l

fCount $=0$;

fVelocityTotal $=0$;

// Add a measurement to the box datum.

void Add (REAL velocity)

i

fCount $+=1$;

fVelocityTotal $+=$ velocity;

1/ Return the count.

UINT GetCount () const

1

了

return fCount;

// Return the velocity total.

REAL GetVelocityTotal() const

l

1

return fVelocityTotal:

private:

// Each box has a vehicle count. 


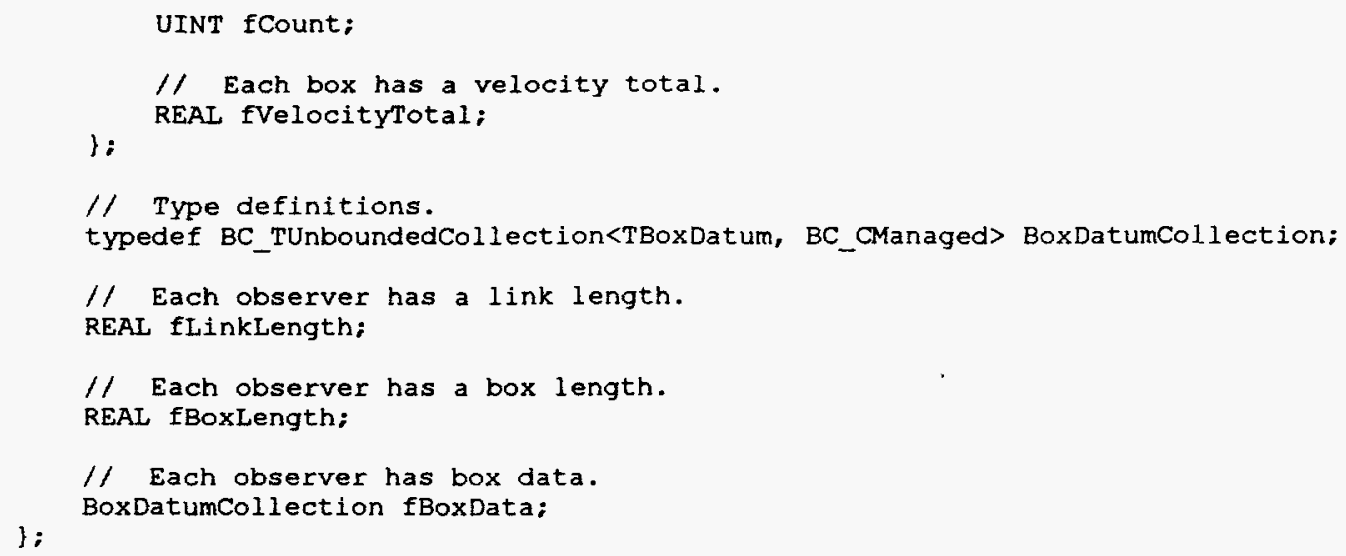

"endif // TRANSIMS_OUT_LINKSPACEOBSERVER

\section{LinkSpaceObserver.C}

$1 /$ Project: TRANSIMS

// Subsystem: Simulation Output

// \$RCSfile: LinkSpaceobserver. $C, v \$$

// SRevision: 0.2 \$

// \$Date: 1996/06/20 14:32:15 \$

$/ /$ SState: Rel \$

// \$Author: bwb \$

// U.S. Government Copyright 1996

// All right reserved

$1 /$ Include TRANSIMS header files.

\#include "OUT/Linkspaceobserver.h"

"include "OuT/SummaryProcessor.h"

"include "OUT/Record.h"

\#include "OuT/Names.h"

// Construct a link space observer.

TOutLinkSpaceObserver: : TOutLinkSpaceObserver (OutObserverId id)

: TOutObserver(id)

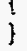

// Destroy a link space observer.

TOutLinkSpaceobserver: : TOutLinkSpaceObserver()

t

// Return whether the observer has been initialized.

bool TOutLinkSpaceobserver: IsInitialized() const

1

\}

return !fBoxData.IsEmpty();

1/ Clear the box data for the observer.

void TOutLinkSpaceobserver: :ClearBoxData ()

l

fBoxData.Clear();

for (int $i=0$; $i$ <LinkLength / fBoxLength; ++i)

l fBoxData.Append (TBoxDatum()):

$/ /$ Set the link id.

void TOutLinkSpaceobserver: :SetLink (NetLinkId id)

l

GetRecord().SetField(kLinkField, id); 
$1 /$ Set the link, box, and cell lengths.

void TOutlinkspaceobserver: : Setlengths (REAL linkLength, REAL boxLength, REAL \{ celliength)

if (celliength $==0$ )

else 1 fLinkLength $=$ linkLength;

fLinkLength $=$ cellLength $*$ int $(1$ inkLength $/$ cellLength $)$;

if (fLinkLength $==0$ )

1

flinkLength $=$ cellLength;

fBoxLength $=$ boxLength;

ClearBoxData();

$1 /$ Set the departure node id.

void TOutLinkSpaceObserver: : SetNode (NetNodeId id)

I

1

GetRecord().SetField(kNodeField, id):

$1 /$ Add a vehicle.

void TOutLinkSpaceobserver: :AddVehicle\{REAL distance, REAL velocity)

\{

const int $1=$ int ( (fLinkLength - distance) $/$ fBoxLength):

\}

fBoxData[i]. Add (velocity):

11 Report the observations to the specified processor.

void TOutLinkSpaceobserver: :Reportobservations (TOutProcessor \& processor)

for (int $i=0$; $i<$ flinktength / fBoxLength; + +i)

TOutRecord\& record = GetRecord ();

record.SetField(kDistanceField, fLinkLength - i * fBoxLength);

record.SetField(kCountField, fBoxData[i].GetCount()):

record.SetField(kSumField, fBoxData[i]. GetVelocityTotal());

1

((TOutSummaryProcessor\&) processor).RecordSpace(*this):

I

\section{TOutLinkTimeObserver Class}

\section{LinkTimeObserver.h}

1/ Broject: TRANSIMS

$1 /$ Subsystem: Simulation Output

// SRCSfile: LinkTimeObserver.h,v \$

$1 /$ \$Revision: 0.1 \$

// SDate: 1996/06/19 17:30:28 \$

// \$State: Stab \$

1/ \$Author: bwb \$

// U.S. Government Copyright 1996

// All right reserved

\#ifndef TRANSIMS OUT LINKTIMEOBSERVER \#define TRANSIMS_OUT_LINKTIMEOBSERVER

1/ Include Booch Components header files.

\#include <BCstoreM.h>

$\#$ include $\langle B C C o 11$ U. $h>$

// Include TRANSIMS header files.

\#include "OUT/Observer.h" 


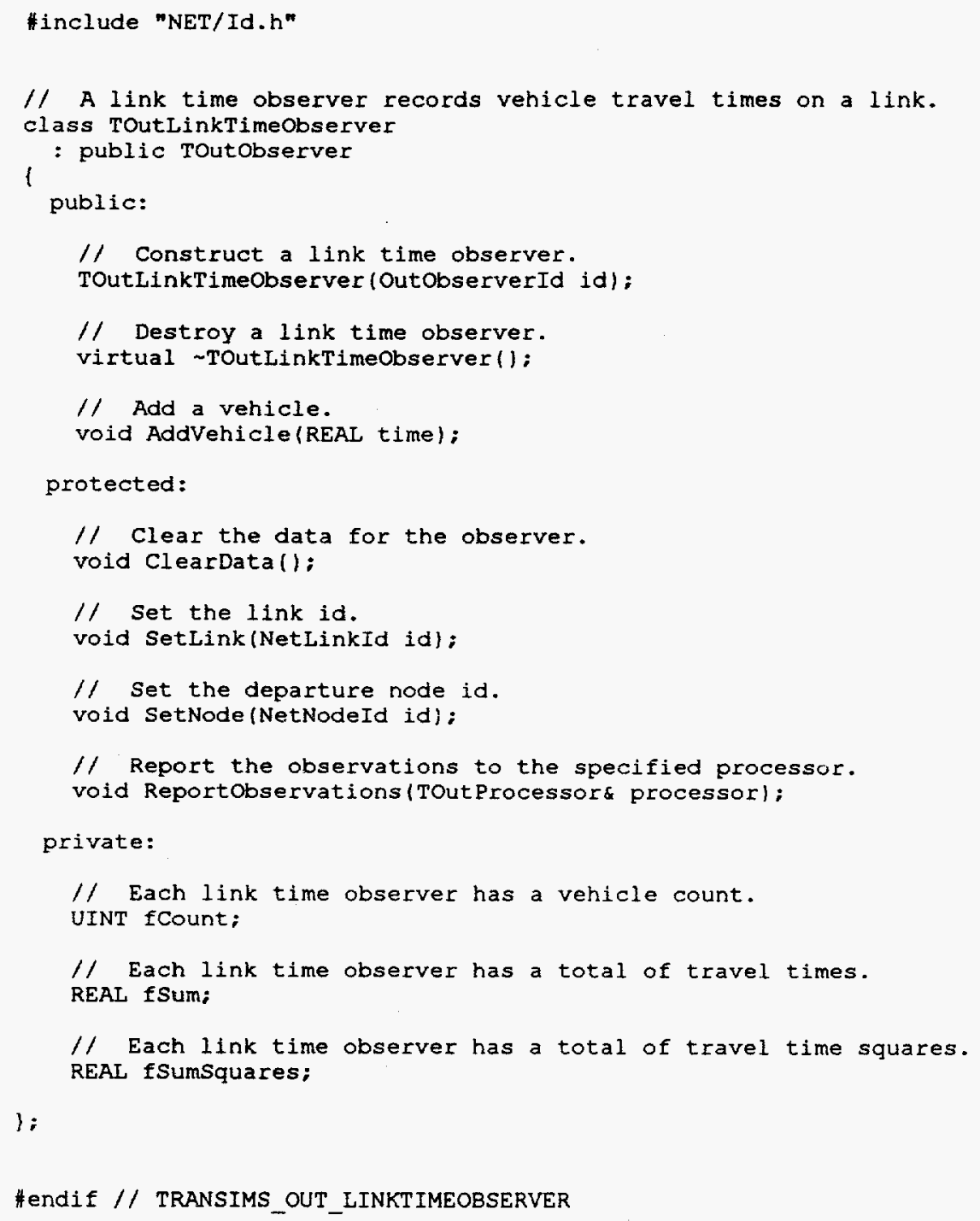

\section{LinkTimeObserver.C}

$/ /$ Project: TRANSIMS

// Subsystem: Simulation Output

// \$RCSfile: LinkTimeObserver.C, $v \$$

// \$Revision: $0.2 \$$

// \$Date: 1996/06/20 14:32:15 \$

$1 /$ \$state: Rel \$

$1 /$ \$Author: bwb \$

$/ /$ U.S. Government Copyright 1996

// Ail right reserved

// Include TRANSIMS header files. "include "OUT/LinkTimeobserver.h" \#include "OUT/SummaryProcessor.h" \#include "OUT/Record.h"

\#include "OUT/Names.h"

// Construct a link time observer.

TOutLinkTimeObserver: : TOutLinkTimeobserver (OutobserverId id)

: Toutobserver(id),

fCount $(0)$.

fSum(0),

fSumSquares $(0)$ 


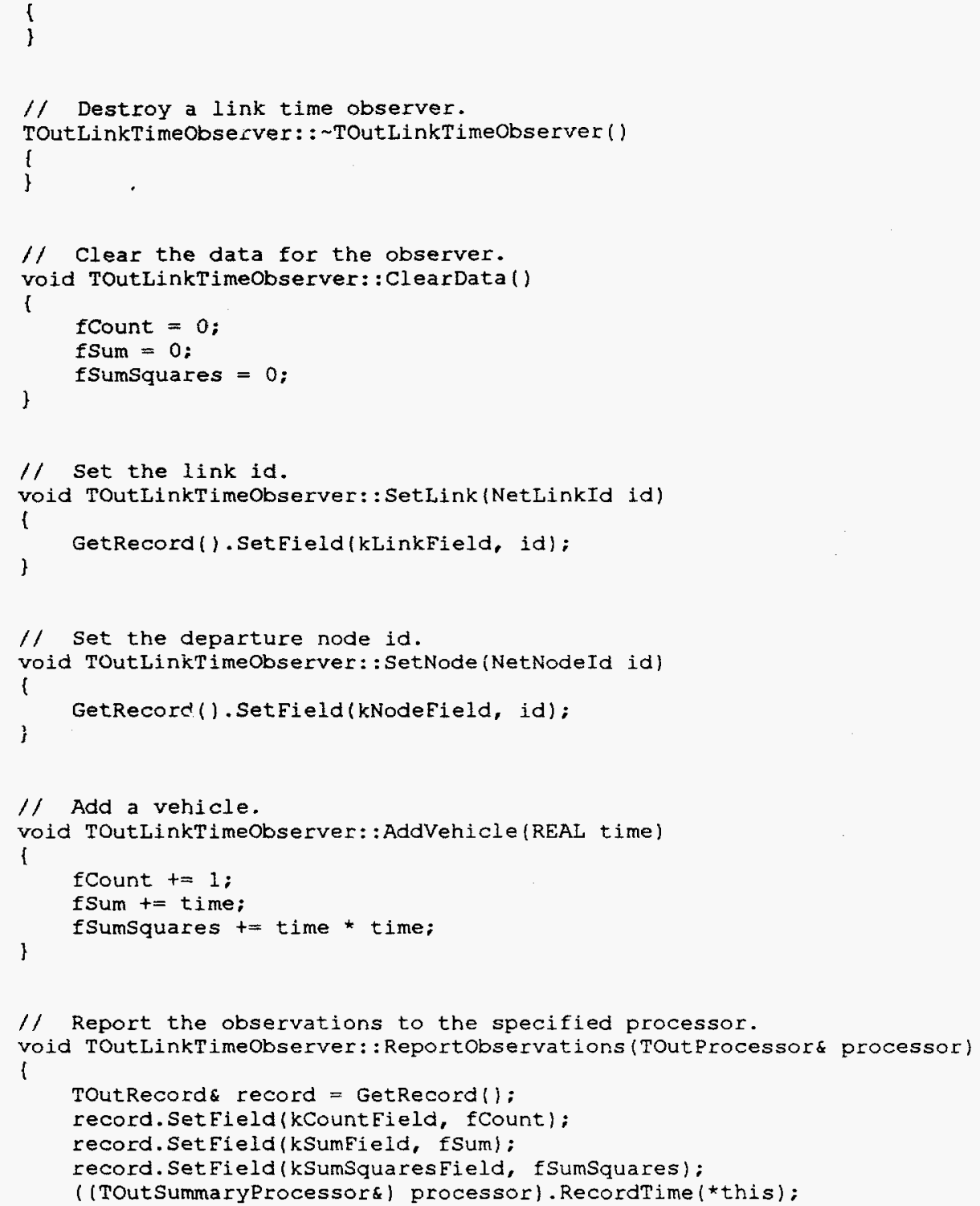

\section{N. TOutNodeEvolutionObserver Class}

\section{NodeEvolutionObserver.h}

$1 /$ Project: TRANSIMS

// Subsystem: simulation Output

// \$RCSfile: NodeEvolutionobserver. $h, v \$$

// SRevision: 0.2 \$

// \$Date: 1996/06/19 17:32:17 \$

$1 /$ \$State: Stab $\$$

// SAuthor: bwb \$

// U.S. Government Copyright 1995

$/ /$ All right reserved

\#ifndef TRANSIMS OUT NODEEVOLOBS

*define TRANSIMS OUT NODEEVOLOBS

// Include TRANSIMS header files. \#include "Out/Observer.h" 
// A node evolution observer observes evolving data related to nodes. class TOutNodeEvolutionobserver

I : public TOutobserver

public:

/ Construct a node evolution observer.

TOutNodeEvalutionobserver (OutobserverId id):

$1 /$ Destruct a node evolution observer.

)

virtual TOutNodeEvolutionObserver ();

\#endif // TRANSIMS_OUT_NODEEVOLOBS

\section{NodeEvolutionObserver.C}

// Project: TRANSIMS

// Subsystem: Simulation Output

// \$RCSfile: NodeEvolutionobserver.C, $\mathrm{s} \$$

// \$Revision: 0.2 \$

// SDate: 1996/06/19 17:31:59 \$

$1 /$ \$State: Stab \$

$1 /$ \$Author: bwb \$

$/ /$ U.S. Government Copyright 1995

$/ /$ All right reserved

// Include TRANSIMS header files.

\#include "OUT/NodeEvolutionObserver.h"

// Construct a node evolution observer.

TOutNodeEvolutionObserver: : TOutNodeEvolutionobserver (OutobserverId id)

: Toutobserver(id)

// Destruct a node evolution observer.

TOutNodeEvolutionObserver: : TOutNodeEvolutionObserver() l

j

\section{o. TOutObserver Class}

1. Observer.h

/ Project: TRANSIMS

1 Subsystem: Simulation Output

/) \$RCsfile: Observer.h, $\mathrm{s}$ \$

// SRevision: $0.4 \$$

// \$Date: 1996/06/19 17:33:26\$

// \$state: Stab \$

// SAuthor: bwb \$

// U.S. Government Copyright 1995

// All right reserved

\#ifndef TRANSIMS OUT OBSERVER

\#define TRANSIMS_OUT_OBSERVER

// Include TRANSIMS header files.

"include "GBL/Globals.h"

"include "OUT/Id.h"

"include "OUT/Record.h" 
// Include Booch Component header files.

\#include "BCStoreM.h"

\#include "BCMapU.h"

// Eorward declarations.

class ToutProcessor;

// An observer converts data from the object to which it is attached

$1 /$ into the generic form understood by the output subsystem.

class TOutobserver

l

public:

// Type definitions

typedef BC_TUnboundedMap<TOutProcessor*, TOutObserver*, 1U, BC_CManaged> ObserverMap;

typedef BC_TMapActiveIterator<TOutProcessor*, TOutObserver ${ }^{\star}>$ observerMapIterator:

// Construct an observer.

TOutObserver (OutObserverId id);

$1 /$ Destruct an observer.

virtual TOutobserver():

// An observer has an observe function for noting the values of data

// members of interest in the observed object.

$1 /$ This virtual function in the basic representation must be

$1 /$ overridden in the view.

virtual void Observe(const void* object, const TOutProcessor processor) = 0 ;

// Return the observer's id.

OutObserverId GetId ():

const OutObserverId GetId() const;

/1 Return the associated record.

TOutRecord\& GetRecord ();

const TOutRecord\& GetRecord() const:

$/ /$ Return the next unused observer id. static OutObserverId GetNextId(1):

protected:

// Define the record's time.

void SetTime (REAL time);

private:

// Do not allow observers to be copied.

TOutobserver (const TOutobserver\&) i)

I! Do not allow observers to be assigned.

TOutObserver\& operator= (const TOutObserver\&) (return *this;)

$1 /$ An observer has an id.

OutobserverId fId;

// An observer has an output record.

TOutRecord fRecord;

// Keep track of the next observer id.

; ; static OutobserverId fNextId;

\#endif // TRANSIMS_OUT_OBSERVER

\section{Observer.C}

// Project: TRANSIMS 
// Subsystem: Simulation Output

// \$RCSfile: Observer.C, $v \$$

$1 /$ SRevision: 0.5 \$

$/ /$ \$Date: 1996/06/19 17:32:51 \$

// \$State: Stab \$

$1 /$ \$Author: bwb \$

// U.S. Government Copyright 1995

// All right reserved

// Include TRANSIMS header files.

"include "OUT/Observer.h"

\#include "OUT/Names.h"

1/ Include $C$ header files.

\#include <stddef. h>

$1 /$ Keep track of the next observer id.

OutObserverId TOutObserver: $:$ NextId $=1000$;

// Construct an observer.

TOutObserver: : TOutObserver (OutObserverId id)

: fId (id)

fRecord ()

1

// Destruct an observer.

Toutobserver: : - TOutObserver()

(

// Return the observer's id.

OutObserverId TOutObserver: :GetId()

l

return erd;

const OutobserverId TOutobserver: :GetId() const

return fId;

// Return the associated record.

TOutRecord\& TOutobserver: :GetRecord!)

I

return fRecord;

const TOutRecord\& TOutObserver: GetRecord ( ) const

l

return fRecord:

// Return the next unused observer id.

OutObserverId TOutObserver: :GetNextId (1)

i

return fNextId++;

// Define the record's time.

void TOutobserver: SetTime(REAL time)

\{

GetRecord().SetField(kTimeField, time); 


\section{P. TOutProcessor Class}

\section{Processor.h}

// Project: TRANSIMS

// Subsystem: Simulation Output

// \$RCSfile: Processor.h,v \$

// \$Revision: 0.4 \$

$1 /$ \$Date: 1996/06/19 17:34:39 \$

// \$State: Stab \$

// \$Author: bwb \$

// U.S. Government Copyright 1995

// All right reserved

\#ifndef TRANSIMS OUT PROCESSOR \#define TRANSIMS_OUT_PROCESSOR

// Include TRANSIMS header files.

\#include "GBL/Globals.h"

\#include "OUT/Id.h"

\#include "OUT/Generalspecification.h"

// An output processor coordinates the processing of domain information

$/ /$ into a domain-independent representation that is filtered and summarized

// before storage.

class TOutProcessor

I

public:

// Processor types.

enum EProcessorType \{kEvolutionProcessor, kEventProcessor, kSummaryProcessor\};

// Construct a processor.

TOutProcessorloutProcessorId id, const TOutGeneralspecification specification);

$/ /$ Destruct a processor.

virtual T TOutProcessor():

// Return the processor type.

virtual EProcessorType GetProcessorType $($ const $=0$;

// Begin recording output for this time.

virtual void Recordoutput (REAL time) $=0$

// Return the processor's id.

OutProcessorId GetId();

const OutProcessorId GetId() const:

// Return the nex unused processor id.

static OutProcessorId GetNextId():

// Return general specification.

ToutGeneralSpecifications GetGeneralspecification ();

const TOutGeneralspecification GetGeneralspecification() const:

private:

// Do not allow processors to be copied.

//TOutProcessor (const TOutProcessor\&) [)

If Do not allow processors to be assigned.

TOutProcessor\& operator $=$ (const TOutProcessor\&) (return *this;)

1/ A processor has an id.

OutProcessorId fId;

// A processor has a general output specification.

TOutGeneralSpecification fGeneralspecification; 


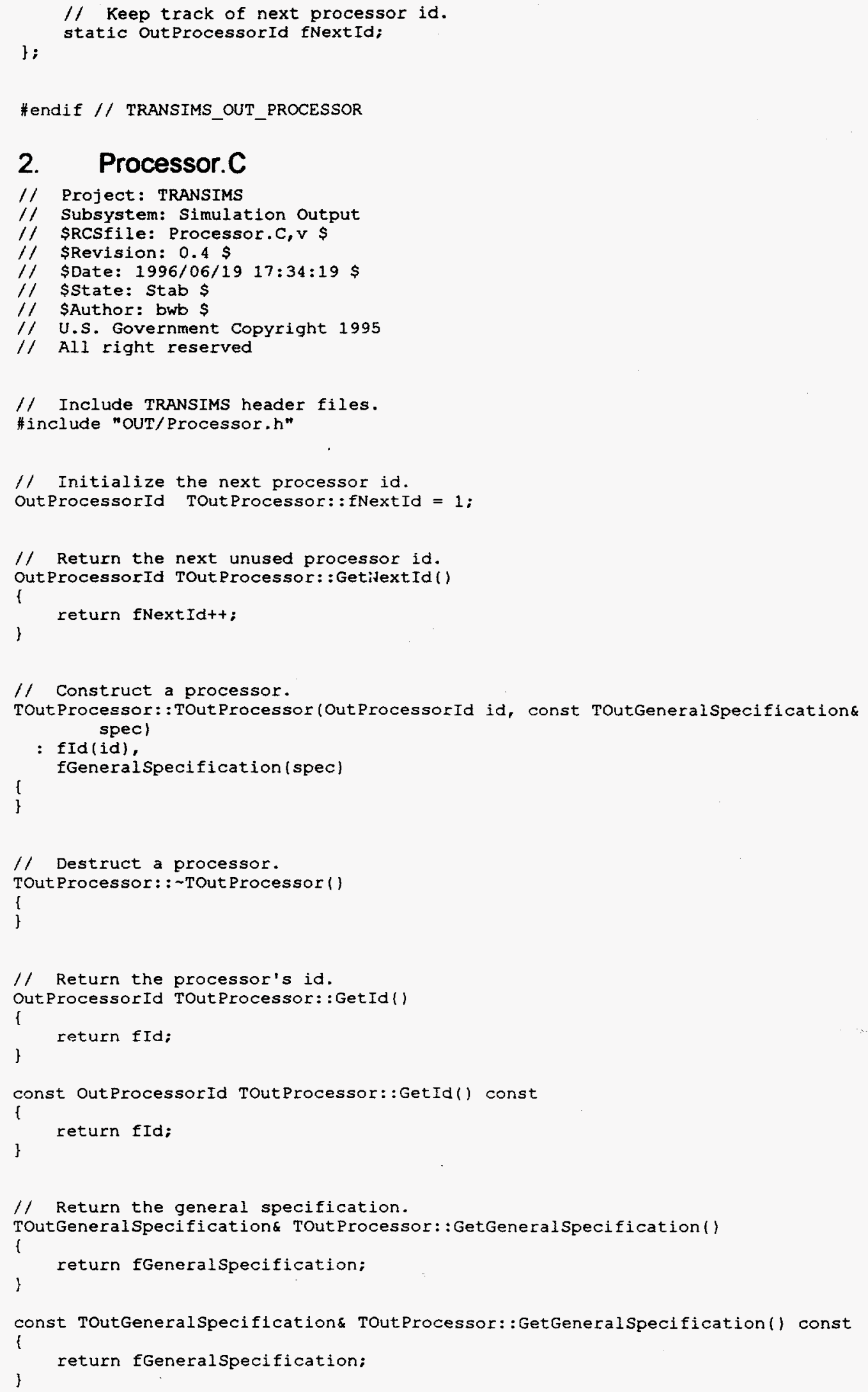




\section{Q. TOutRecord Class}

\section{Record.h}

$1 /$ Project: TRANSIMS

// Subsystem: Simulation Output

// SRCSfile: Record.h,v s

// SRevision: $0.6 \$$

// SDate: 1996/06/19 17:35:23 \$

// SState: Stab \$

$1 /$ \$Author: bwb \$

// U.S. Government Copyright 1995

// All rights reserved

\#ifndef TRANSIMS_OUT_RECORD

\#define TRANSIMS_OUT_RECORD

// Include Booch Components header files.

\#include <BCStoreM.h>

\#include <BCMapU.h>

// Include DBtools.h+t header files.

\#include $\langle\mathrm{rw} / \mathrm{db} / \mathrm{val}$ ue.h〉

// Fix for Booch Maps.

inline int operator! $=$ (const RWDBValue\& $a$, const RWDBValue $b$ )

1

\}

return !a.isEqual (s.b):

$/ /$ Include TRANSIMS header files.

\#include <GBL/Globals.h>

// This class is used for storing the values of a collection of fields. class TOutRecord

I

public:

$1 /$ Type definitions.

typedef BC_TUnboundedMap<const string, RWDBValue, 30U, BC_CManaged> FieldMap;

typedef BC_TMapActiveIterator<const string, RWDBValue> FieldMapIterator;

// Field types.

enum Type $\{$ kNoType = RWDBValue: : NoType, kChar = RWDBValue: :Char,

kUnsignedChar = RWDBValue: :UnsignedChar, kShort = RWDBValue: : Short, kUnsignedShort $=$ RWDBValue: : UnsignedShort, kInt $=$ RWDBValue: : Int, kUnsignedInt = RWDBValue: :UnsignedInt, kLong = RWDBValue: :Long, kUnsignedLong = RWDBValue: : UnsignedLong, kEloat = RWDBValue:: Eloat, kDouble = RWDBValue: $:$ Double, kString = RWDBValue: $:$ String ;

$1 /$ Construct a record.

TOutRecord ():

1/ Make a copy of the given record.

// TOutRecord(const TOutRecord\& record);

1/ Make the record a copy of the given.

// TOutRecord\& operator=(const TOutRecords record):

$1 /$ Set the value of the specified field.

void SetField(const string\& field, char value);

void SetField(const string\& field, unsigned char value)

void SetField(const string field, short value);

void setfield(const string\& field, unsigned short value);

void SetField(const string f field, int value);

void SetField(const string field, unsigned int value); 
void SetField(const strings field, long value):

void SetField(const string\& field, unsigned long value):

void SetField(const string field, float value):

void SetField(const string\& field, double value);

void SetField(const string field, const string valuel:

void setField(const string field):

$1 /$ Get the value of the specified field.

void Getfield(const string\& field, char\& value) const;

void Getfield(const string\& field, unsigned char\& value) const;

void GetField(const string\& field, short valuel const;

void GetField(const stringe field, unsigned short value) const;

void GetField(const string\& field, int \& value) const;

void GetField(const stringe field, unsigned inta value) const;

void GetField(const string\& field, long\& value) const;

void GetField(const string\& field, unsigned long\& value) const;

void GetEield(const string\& field, float \& value) const;

void GetField(const string field, double\& value) const:

void GetField(const string\& field, string\& value) const;

// Return the type of the specified field.

Type GetType (const string field) const:

$1 /$ Return the map for the record.

FieldMap\& GetMap ();

const FieldMap\& GetMap() const:

// Return an iterator for the record's map.

FieldMapIterator GetIterator() const;

private:

// Each record has a field map holding the current values of the fields.

FieldMap fFields;

$1:$

"Eendif // TRANSIMS_OUT_RECORD

\section{Record.C}

// Project: TRANSIMS

// Subsystem: Simulation output

// SRCSfile: Record.C, $\mathrm{V}$ \$

// SRevision: $0.5 \$$

// SDate: 1996/06/19 17:35:17\$

// Sstate: Stab $\$$

$1 /$ SAuthor: bwb $\$$

// U.S. Government Copyright 1995

// All rights reserved

// Include TRANSIMS header files.

$\#$ include <OUT/Record.h〉

(/ Define the hash function for strings.

static BC_Index StringHashvalue (const string\& $s$ )

f

return Hashvalue(s):

// Construct a record.

TOutRecord: : TOutRecord ()

: fFields (StringHashvalue)

!

// Set the value of the specified field.

void TOutRecord: SetEield(const string field, char value)

\{ 


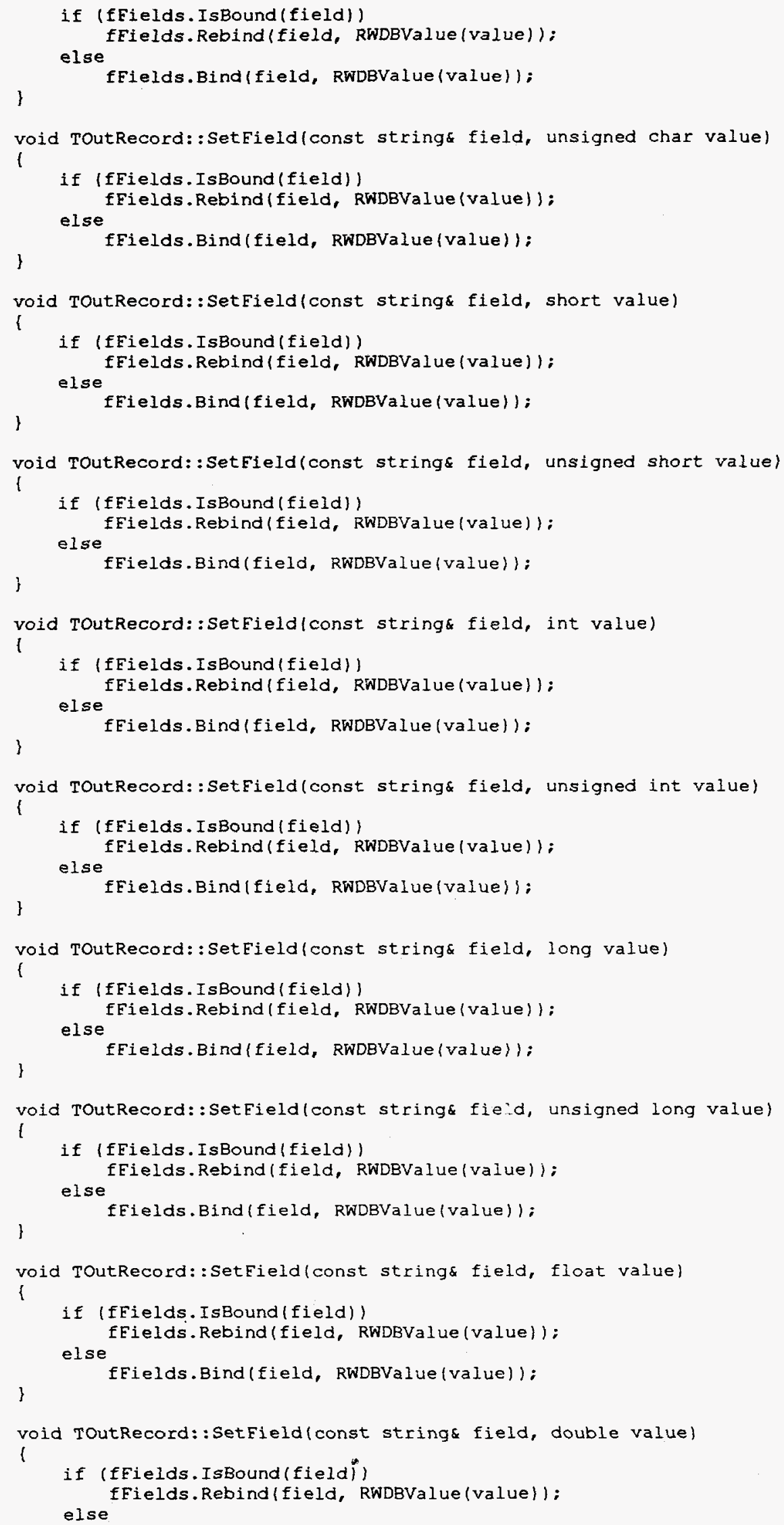




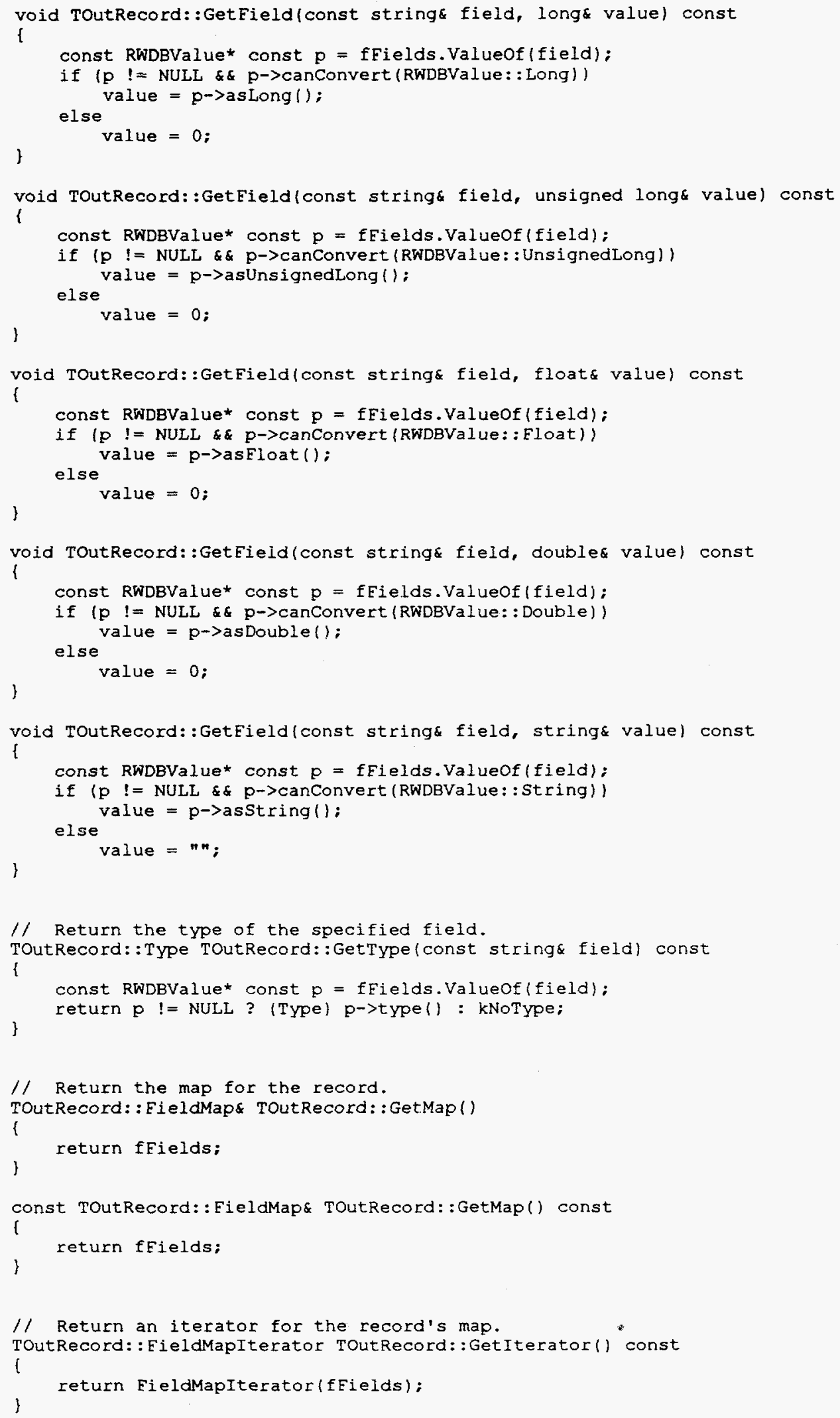




\section{R. TOutRetriever Class}

\section{Retriever.h}

$1 /$ Project: TRANSIMS

// Subsystem: Simulation Output

// \$RCSfile: Retriever.h,v \$

// \$Revision: 0.3 \$

// \$Date: 1996/06/19 17:36:07 \$

// \$State: Stab \$

// SAuthor: bwb \$

// U.S. Government Copyright 1995

//. All rights reserved

\#ifndef TRANSIMS_OUT_RETRIEVER \#define TRANSIMS OUT RETRIEVER

// Include TRANSIMS header files.

"include <GBL/Globals.h>

\#include <NET/Network.h>

\#include <OuT/Generalspecification.h>

\#include <OUT/Record.h>

Hinclude <OUT/Storage.h>

\#include <OUT/Writer.h>

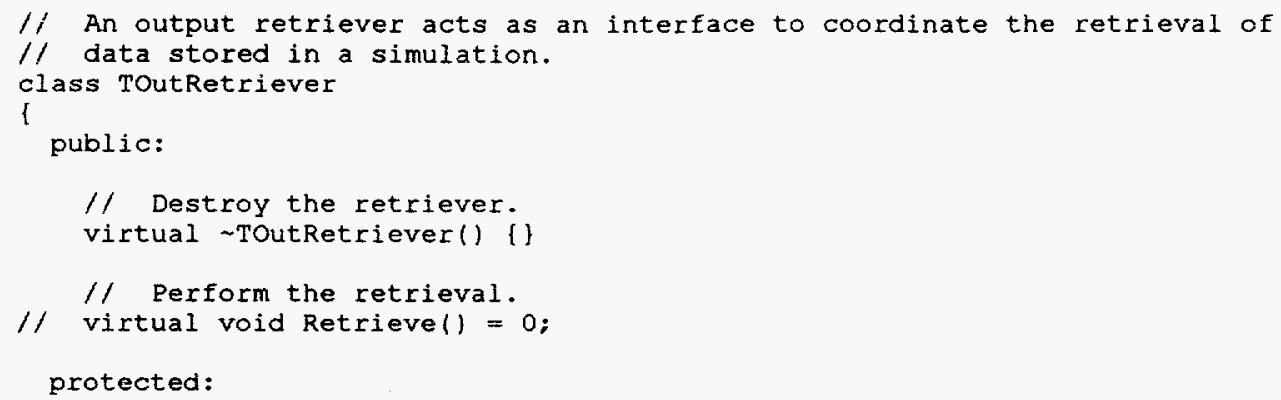


\# endif // TRANSIMS_OUT_RETRIEVER

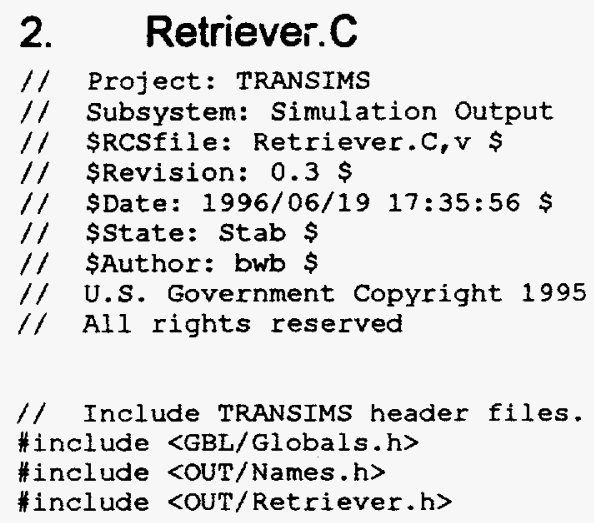

// Subsystem: Simulation Output

// SRCSfile: Retriever.C, $v$ \$

// SRevision: 0.3 \$

// \$Date: 1996/06/19 17:35:56 \$

// \$State: Stab \$

// \$Author: bwb \$

// U.S. Government Copyright 1995

// All rights reserved

$1 /$ Include TRANSIMS header files.

\#include <GBL/Globals.h>

\#include <OUT/Names.h〉

\#include <ouT/Retriever.h>

// Construct a retriever based on the given specification.

TOutRetriever: :TOutRetriever(const TOutGeneralSpecification specification, const TNetNetwork* network)

: fGeneralspecification (specification). fNetwork (network)

1

// Return the general specification.

TOutGeneralSpecificationd TOutRetriever: GetGeneralSpecification()

1

return fGeneralspecification:

const TOutGeneralSpecification TOutRetriever: GetGeneralSpecification() const 1

\}

return fGeneralspecification:

// Return the network, if any.

const TNetNetwork* TOutRetriever::GetNetwork() const

1

return fNetwork;

$1 /$ Retrieve data from the specified storage and put it in the specified

$/ /$ writer, sorting it if indicated, and performing time and space filtering

$1 /$ if indicated.

void TOutRetriever: :BasicRetrieve(TOutStorage storage, TOutWriter\& writer, bool sort, bool filterl

f

// Allocate caches.

const int $\mathrm{nh}=$ storage.GetHosts (). Extent ();

TOutStorage: : HostHandle* handleCache $=$ new TOutStorage: :HostHandle $[\mathrm{nh}]$;

TOutRecord* recordCache $=$ new TOutRecord $[\mathrm{nh}]$;

$1 /$ Setup caches.

TOutRecord record;

TOutStorage: :HostSetIterator i(storage.GetHosts());

int $h=0$;

for (i.Reset (); !i.IsDone(); i.Next()) (

const strings host $=* i$. CurrentItem();

storage. Seek (host, Toutstorage: :kBegin);

storage. ReadHeader (host, record):

)

handleCache $[\mathrm{ht+}]=$ storage. GetHostHandle(host):

$/ /$ Setup output. 


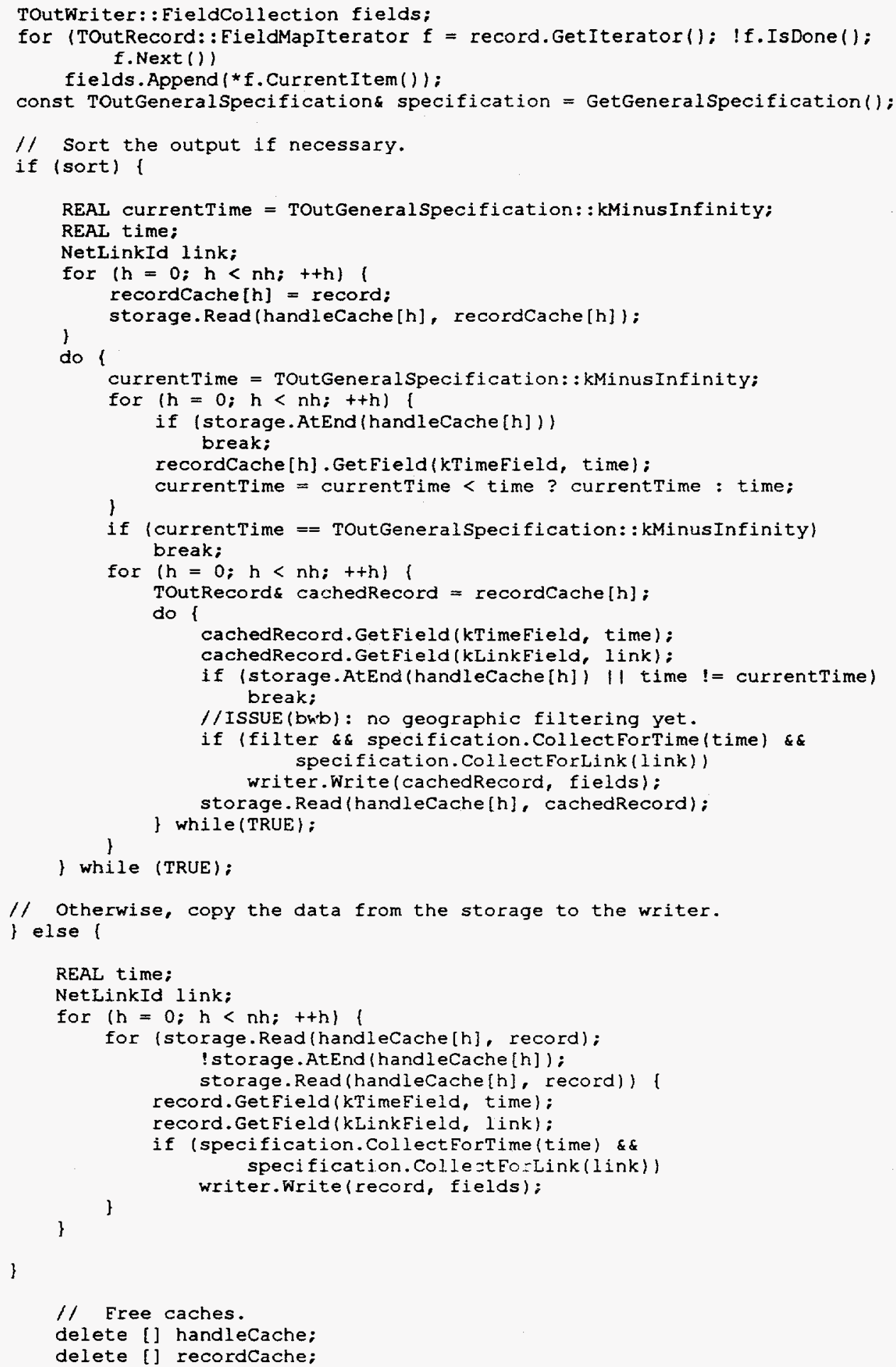

\section{S. TOutSignalCoordinatorEvolutionObserver Class}

\section{SignalCoordinatorEvolutionObserver.h}

// Project: TRANSIMS

// Subsystem: Simulation Output

// SRCSfile: SignalCoordinatorEvolutionObserver.h, v \$ 
$1 /$ SRevision: 0.2 \$

// \$Date: 1996/06/19 17:37:18 \$

// \$State: Stab \$

// SAuthor: bwb \$

// U.S. Government Copyright 1995

// All right reserved

\#ifndef TRANSIMS_OUT_COORDEVOLOBS

"define TRANSIMS_OUT_COORDEVOLOBS

// Include TRANSIMS header files.

"include "OUT/Observer. $h$ "

// A signal coordinator evolution observer observes evolving data related to

$/ /$ signal coordinators.

class ToutSignalCoordinatorEvolutionobserver

1

: public TOutObserver

public:

// Construct a signal coordinator evolution observer.

TOutSignalCoordinatorEvolutionobserver (OutobserverId id);

// Destruct a signal coordinator evolution observer.

\} ;

virtual ToutSignalCoordinatorEvolutionobserver():

\#endif // TRANSIMS_OUT_COORDEVOLOBS

\section{SignalCoordinatorEvolutionObserver.C}

// Project: TRANSIMS

// Subsystem: Simulation Output

// \$RCSfile: SignalCoordinatorEvolutionObserver.C, $v \$$

$1 /$ \$Revision: 0.2 \$

// \$Date: 1996/06/19 17:36:47 \$

$1 /$ \$State: Stab \$

// \$Author: bwb \$

// U.S. Government Copyright 1995

// All right reserved

// Include TRANSIMS header files.

\#include "OUT/SignalCoordinatorEvolutionobserver.h"

$/ /$ Construct a signal coordinator evolution observer.

TOutSignalCoordinatorEvolutionobserver: :TOutSignalCoordinatorEvolutionobserver (OutabserverId id)

!

: Toutobserver(id)

$1 /$ Destruct a signal coordinator evolution observer.

TOutSignalCoordinatorEvolutionobserver: : TOutSignalCoordinatorEvolutionobserver()

1

\section{T. TOutSignalizedControlObserver Class}

\section{SignalizedControlObserver.h}

$1 /$ Project: TRANSIMS

// Subsystem: Simulation Output

// SRCSfile: SignalizedControlobserver.h,v $\$$

// SRevision: 0.2 \$

// SDate: 1996/06/19 17:38:02 \$ 


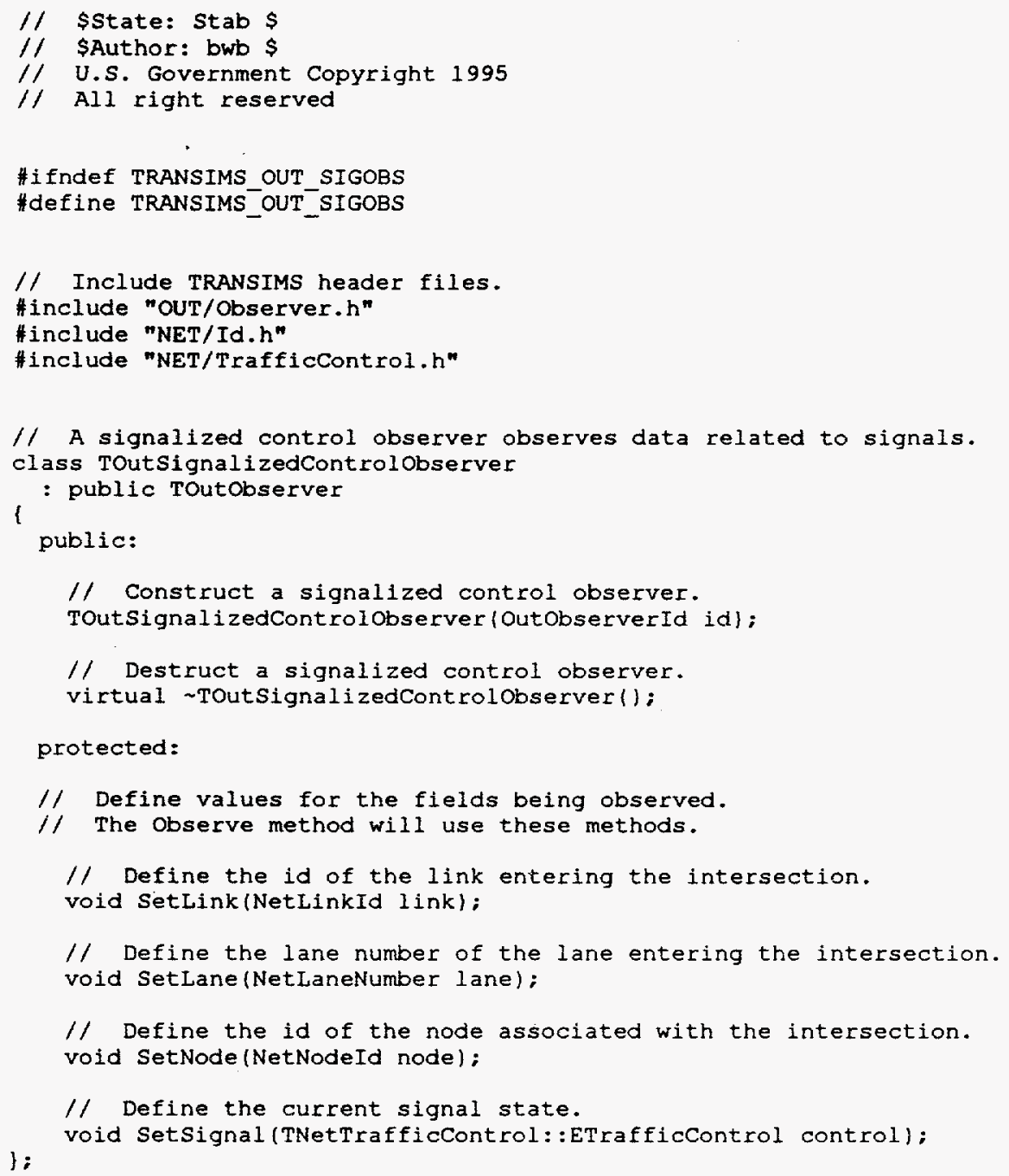

\#endif // TRANSIMS_OUT_SIGOBS

\section{SignalizedControlObserver.C}

// Project: TRANSIMS

$1 /$ Subsystem: Simulation Output

// \$RCSfile: SignalizedControlobserver.C,v $\$$

$1 /$ SRevision: $\hat{v} .2$ \$

// \$Date: 1996/06/19 17:37:46 \$

$1 /$ \$State: Stab $\$$

// \$Author: bwb \$

// U.S. Government Copyright 1995

// All right reserved

// Include TRANSIMS header files.

\#include "OUT/SignalizedControlobserver.h"

\#include "OUT/Record.h"

"include "OUT/Names. $h$ "

$1 /$ Construct a signalized control observer.

TOutSignalizedControlobserver: : TOutSignal izedControlobserver (OutobserverId id) : Toutobserver(id)

\{

SetTime $(-99$.$) :$

SetLink $(-99)$ : 


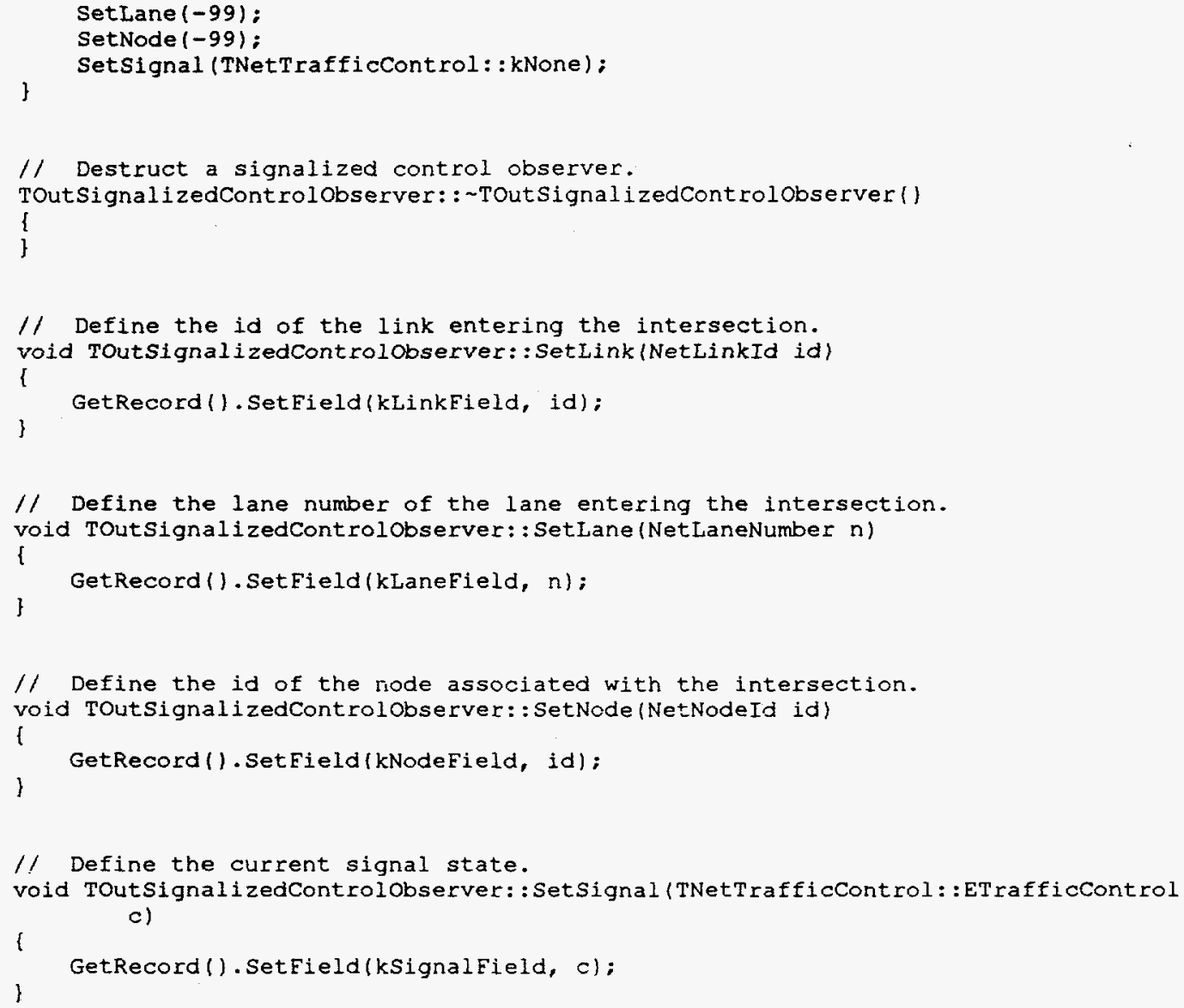

\section{U. TOutSpecificationReader Class}

\section{SpecificationReader.h}

$1 /$ Project: TRANSIMS

1/ Subsystem: Simulation Output

// SRCSfile: SpecificationReader,h, $v \$$

1/ SRevision: $0.3 \$$

1/ \$Date: 1996/06/19 17:38:30 \$

// SState: stab \$

$1 /$ SAuthor: bwb $\$$

// U.S. Government Copyright 1995

// All rights reserved

\# I fndef TRANSIMS OUT SPECIFICATIONREADER \#define TRANSIMS_OUT_SPECIFICATIONREADER

// Include TRANSIMS header files.

\#include <GBL/Globals.h>

\#include <DBS/Table.h>

// A specification reader reads an output specification from the database. class TOutSpecificationReader

1

public:

// Construct a reader for the specified tables.

TOutspecificationReader (TDbTable generalTable, TDbTable nodeTable, TDbTable linkTable): 


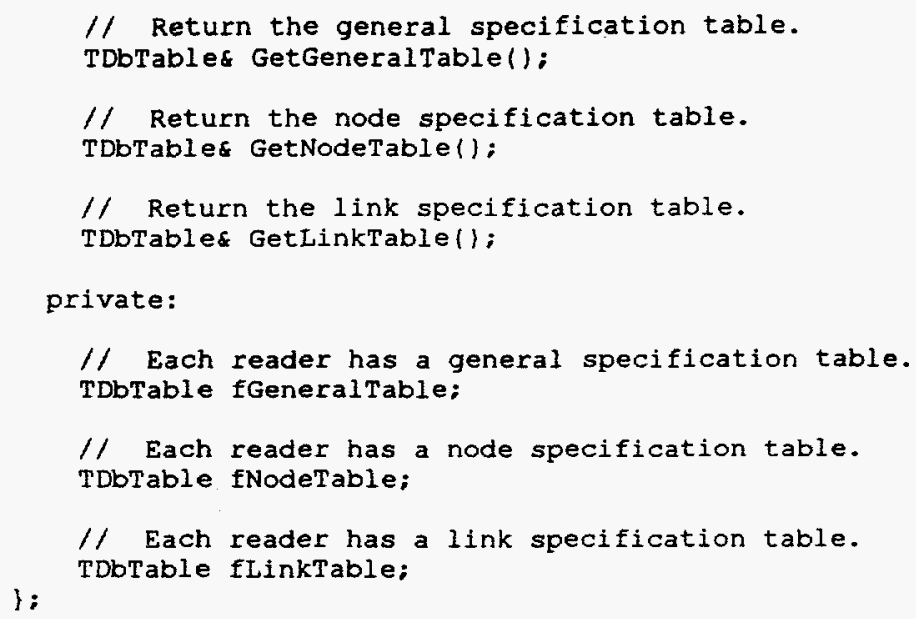

\#endif // TRANSIMS_OUT_SPECIFICATIONREADER

\section{SpecificationReader.C}

// Project: TRANSIMS

// Subsystem: Simulation Output

// \$RCSfile: SpecificationReader.C, $v$ \$

// SRevision: 0.3 \$

// SDite: 1996/06/19 17:38:22 \$

// \$State: Stab $\$$

// \$Author: bwb \$

// U.S. Government Copyright 1995

// All rights reserved

$1 /$ Include TRANSIMS header filles.

\#include <OUT/SpecificationReader.h>

// Construct a reader for the specified tables.

TOutSpecificationReader: :TOutSpecificationReader (TDbTable generalTable, TDbTable nodeTable, TDbTable inkTable)

: fGeneralTable (generalTable),

fNodeTable (nodeTable),

flinkTable (linkTable)

\{

// Return the general specification table.

TDbTable\& TOutSpecificationReader::GetGeneralTable()

1

return fGeneralTable;

// Return the node specification table.

TDbTable \& ToutSpecificationReader: :GetNodeTable()

1

1

return fNodeTable:

$1 /$ Return the link specification table.

TDbTable\& TOutSpecificationReader: :GetLinkTable()

1

1

return flinkTable; 
/f Return the host handle.

Hosthandle Gethosthandle(const string\& host) const;

// Return the current offset for the specified host file.

long Getoffset() const:

long Getoffset (const string\& host) const;

long Getoffset (Hosthandle host) const;

$/$ Return whether an end-of-file has occured for the specified host file. bool AtEnd () const:

bool AtEnd (const string\& host) const;

bool AtEnd(HostHandle host) const;

// Position the specified host file to the given location.

void Seek (long position = kBegin);

void Seek (const string h host, long position $=k$ Begin):

void Seek (Hosthandle host, long position $=\mathrm{kBegin}$ );

// Write the given record on the specified host.

void Write(const ToutRecord\& record);

vold Write (const string\& host, const ToutRecord\& record);

void Write (Hosthandle host, const TOutRecord\& record);

// Read the given record on the specified host. Return whether a record

// was available for reading.

bool Read(TOutRecord\& record):

bool Read(const string\& host, TOutRecord\& record);

bool Read (Hosthandle host, TOutRecord\& record);

// Write the given record header on the specified host.

void WriteHeader(const TOutRecord\& record);

void WriteHeader (const string\& host, const ToutRecord\& record);

void WriteHeader (Hosthandle host, const TOutRecords record);

1/ Read the given record header on the specified host. Return whether a

$1 /$ record was available for reading.

bool ReadHeader (TOutRecord \& record);

bool ReadHeader (const string\& host, ToutRecord\& record):

bool ReadHeader (HostHandle host, TOutRecord\& record);

// Flush any pending operations.

void Elush();

private:

// Type definitions.

typedef BC TUnboundedMap<const string, RWEile*, 30U, BC CManaged> FileMap;

typedef BC_TMapActiveIterator<const string, RWFile*> Ei IeMapIterator;

// Local host name.

static const string fgLocal;

() File suffix.

static const string fgSuffix;

// Do not allow storages to be copied.

TOutstorage (const Toutstorage\&) (\}

// Do not allow storages to be assigned.

TOutstorage operator= (const Toutstorage\&) (return *this;)

// A storage has a root location.

const string fRoot:

1/ A storage has a basic name.

const string fName;

1/ A storage has a file on each host.

;

FileMap fEiles:

\#endif // TRANSIMS_OUT_STORAGE 


\section{Storage.C}

$1 /$ Project: TRANSIMS

$/ /$ Subsystem: Simulation Output

// \$RCSfile: Storage.C,v \$

// SRevision: $0.5 \$$

$/ /$ SDate: 1996/06/19 17:38:48 \$

$/ /$ \$state: Stab $\$$

// \$Author: bwb \$

// U.S. Government Copyright 1995

// All rights reserved

// Include Booch Component header files.

\#include <BCStoreM.h>

Ninclude <BCStacu.h>

$/ /$ Include TRANSIMS header files.

\#include <OUT/Storage.h>

// Define the hash function for strings.

static BC_Index StringHashvalue (const string\& $s$ )

I

1

return Hashvalue (s):

$1 /$ Constants for seek positions.

const long Toutstorage: : kBegin $=0$,

const long TOutStorage: $: k E n d=-1$;

$1 /$ Local host name.

const string TOutStorage: : fgLocal = "local";

$/ /$ File suffix.

const string TOutStorage: :fgSuffix = ".stg":

// Connect the storage with the given root and basic name to the specified

// hosts.

TOutStorage: :TOutStorage (const string\& root, const string\& name, Mode mode)

: fRoot (root),

fName (name).

fFiles (Stringtashvalue)

string modestring;

switch (mode) 1

case kRead:

modestring $=$ "rb"

break;

case kwrite:

modestring $=" w b+"$;

break;

case kDelete:

\}

throw TOutStorageFailure("Deletion not supported.");

RWFile* const file $=$ new RWFile $($ fRoot $+n / n+$ fglocal $+n / n+$ fName + fgsuffix, modestring);

- if (!file->isvalid())

throw TOutStorageFailure("Cannot open file.");

] fFiles.Bind(fglocal, file);

TOutStorage: :TOutStorage(const HostSet\& hosts, const string\& raot, const string\& name, Mode mode)

: fRoot (root),

fName (name),

EEiles (StringHashValue)

\{

string modestring; 


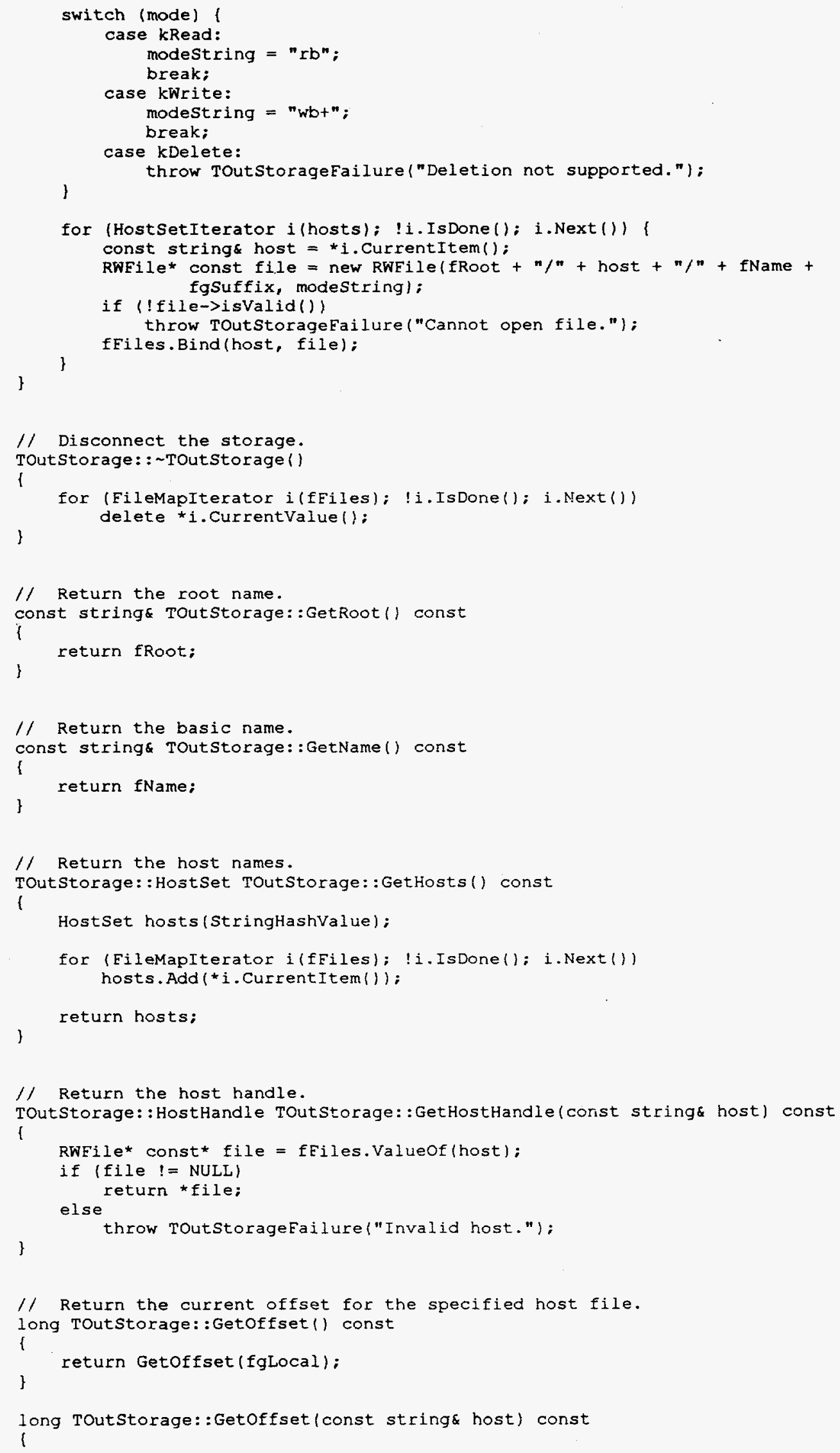




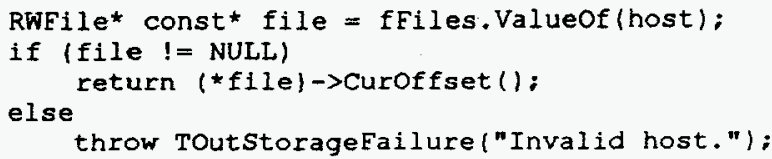




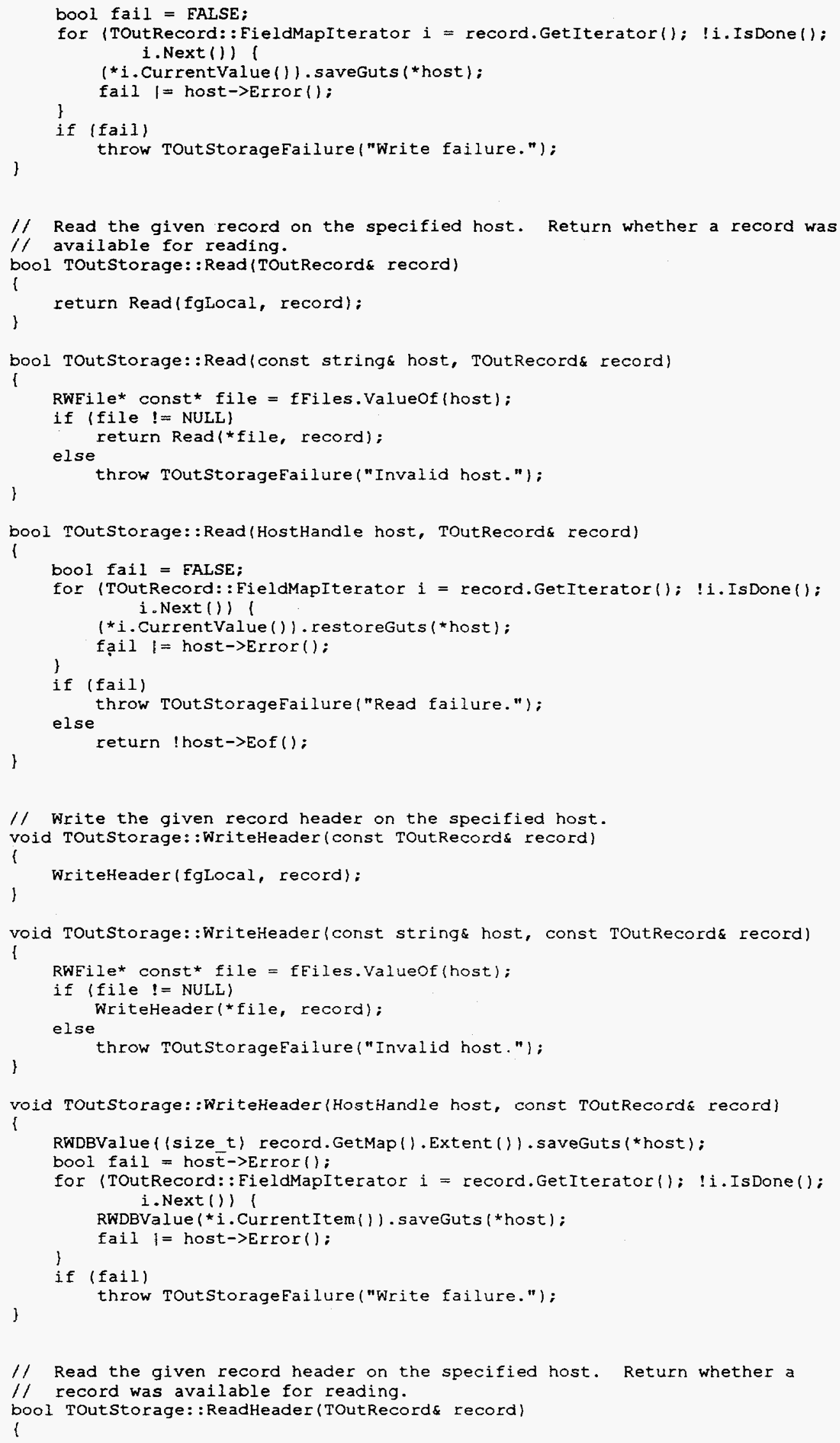


bool TOutstorage: ReadHeader(const string\& host, TOutRecord\& record) \{

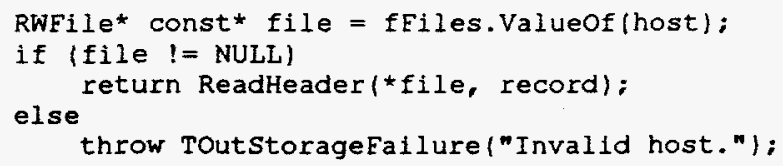

\section{W. TOutSummaryProcessor Class}

\section{SummaryProcessor.h}

$1 /$ Project: TRANSIMS

// Subsystem: Simulation Output

// SRCSfile: SummaryProcessor.h,v $\$$

1/ SRevision: $0.1 \$$

// \$Date: 1996/06/19 17:39:48 \$

/) \$state: Stab \$

1) SAuthor: bwb $\$$

// U.S. Government Copyright 1996

// AlI right reserved

\#i fndef TRANSIMS_OUT_SUMMARYPROCESSOR \#define TRANSIMS_OUT_SUMMARYPROCESSOR

// Include Booch Components header files.

\#include <BCStoreM.h>

\#include <BCSetU.h>

$1 /$ Include TRANSIMS header files.

\#include "OUT/Processor.h"

"include "OUT/Storage. $h$ " 
// Forward declarations.

class TOutObserver;

// An output summary processor deals with collects statistics on the

// simulation.

class ToutSummaryProcessor

: public ToutProcessor

\

public:

// Type definitions.

typedef BC TUnboundedSet<TOutObserver*, $10000 \mathrm{U}, \mathrm{BC}$ CManaged> ObserverSet;

typedef BC_TSetActiveIterator<TOutObserver*> ObserverSetIterator;

// Construct a summary processor.

TOutSummaryProcessorloutProcessorId id, const TOutGeneralspecification\& specificationl:

$1 /$ Destroy a summary processor.

virtual TOutSummaryProcessor():

// Return processor type.

EProcessorType GetProcessorType | const;

$/ /$ Begin recording output for this time step.

virtual void Recordoutput(REAL time):

/f Finish recording output for link space.

virtual void RecordSpace (const Toutobserver observer);

// Finish recording output for link time.

virtual void RecordTime (const TOutobservers observer);

// Return the space observers.

ObserverSet GetSpaceobservers (1);

const ObserverSet\& GetSpaceObservers() const;

// Return the time observers.

ObserverSet\& GetTimeObservers ();

const observerSet\& GetTimeobservers() const;

// Define a space observer.

void AddSpaceobserver(TOutObserver\& observer);

// Define a time observer.

void AddTimeobserver(TOutobservers observer);

// Undefine a space observer.

void RemoveSpaceobserver(TOutobserver\& observer);

// Undefine a time observer.

void RemoveTimeobserver(TOutobserver\& okserver):

private:

// Do not allow summary processors to be copied.

//TOutSummaryProcessor (const ToutSummaryProcessor\&) (\}

// Do not allow summary processors to be assigned.

//TOutSummaryProcessor operator $=$ (const TOutSummaryProcessor $\&$ ) (return

$1 / \quad$ this;

1/ A summary processor has space observers.

ObserverSet fSpaceObservers;

// A summary processor has time observers;

Observerset fTimeObservers;

// An summary processor is connected to a space storage.

TOutStorage fSpacestorage;

// A summary processor is connected to a time storage. 


\section{\#endif // TRANSIMS_OUT_SUMMARYPROCESSOR}

\section{SummaryProcessor.C}

// Project: TRANSIMS

// Subsystem: Simulation Output

// \$RCsfile: SummaryProcessor.,$v \$$

// SRevision: 0.1 \$

// \$Date: 1996/06/19 17:39:17 \$

// \$State: Stab \$

$1 /$ \$Author: bwb \$

// U.S. Government Copyright 1996

$/ /$ All right reserved

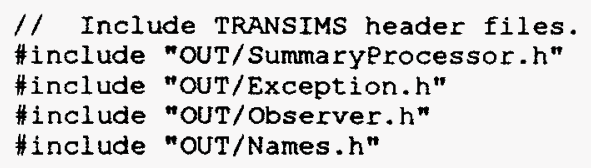


// Finish recording output for link time.

void TOutSummaryProcessor: : RecordTime (const TOutobserver observer)

l

if (fTimestorage.Getoffset() $==0$ )

fTimestorage.WriteHeader(observer.GetRecord (1):

\}

fTimeStorage.Write(observer.GetRecord ());

$1 /$ Return the space observers.

TOutSummaryProcessor: : ObserverSet\& TOutSummaryProcessor::GetSpaceobservers (\}

1

\}

return fSpaceobservers;

const TOutSummaryProcessor: : ObserverSet\&

TOutSummaryProcessor: :GetSpaceObservers () const

1

return fSpaceobservers;

)

$1 /$ Return the time observers.

TOutSummaryProcessor: : ObserverSet \& TOutSummaryProcessor: :GetTimeobservers()

1

\}

return fTimeobservers;

const TOutSummaryProcessor: : ObserverSet\&

TOutSummaryProcessor: :GetTimeobservers ( ) const

I

return fTimeobservers;

了

// Define a space observer.

void TOutSummaryProcessor: :AddSpaceObserver (TOutObserver\& observer)

1

)

fSpaceObservers. Add (\&observer);

$1 /$ Define a time observer.

void TOutSummaryProcessor: :AddTimeobserver (TOutobserver\& observer)

1

)

fTimeobservers. Add (cobserver);

$1 /$ Undefine a space observer.

void TOutSummaryProcessor: :RemoveSpaceObserver!(TOutObserver\& observer)

1

)

fSpaceobservers. Remove (\&observer);

$1 /$ Undefine a time observer.

void TOutSummaryProcessor: : RemoveTimeobserver (TOutObserver observer)

\{

\}

fTimeobservers.Remove (\&observer):

\section{TOutSummaryRetriever Class}

\section{SummaryRetriever.h}

// Project: TRANSIMS

// Subsystem: Simulation Output

$/ /$ SRCSfile: SummaryRetriever.h,v $\$$ 
$1 /$ SRevision: 0.1 \$

// \$Date: 1996/06/19 17:41:04 \$

// \$State: Stab \$

1/ \$Author: bwb \$

// U.S. Government Copyright 1995

// All rights reserved

\#ifndef TRANSIMS OUT SUMMARYRETRIEVER *define TRANSIMS_OUT_SUMMARYRETRIEVER

// Include TRANSIMS header files.

\#include 〈GBL/Globals.h〉

\#include <OUT/Retriever.h>

// An event retriever gets specific summary data from storage and

$/ /$ coordinates its conversion and filtering.

class TOutSummaryRetriever

\{

: public TOutRetriever

public:

// Construct a reader for the specified hosts and given specification,

$1 /$ and network.

TOutSummaryRetriever lconst TOutStorage: :HostSet\& hosts, const TOutGeneralspecification specification, const TNetNetwork* network

$=$ NULI):

$1 /$ Perform the retrieval on the specified writers.

void Retrieve(ToutWriter\& spaceWriter, TOutWriter timeWriter, bool sort = TRUE):

private:

// The retriever is connected to a space storage.

TOutStorage fSpaceStorage:

1/ The retriever is connected to a time storage.

\}

TOutstorage ETimestorage:

\# endif // TRANSIMS_OUT_SUMMARYRETRIEVER

\section{SummaryRetriever.C}

$1 /$ Project: TRANSIMS

1/ Subsystem: Simulation Output

// \$RCSfile: SummaryRetriever.C,v \$

// \$Revision: 0.1 \$

1/ SDate: 1996/06/19 17:40:36 \$

// \$State: Stab \$

1/ \$Author: bwb \$

// U.S. Government Copyright 1996

// A.ll rights reserved

// Include TRANSIMS header files.

"include <OUT/SummaryRetriever.h>

\#include <OUT/Names.h>

$/ /$ Construct a reader for the specified hosts and given specification. TOutSummaryRetriever: : TOutSumma ryRetriever (const TOutStorage: : Host Set\&

hosts, const TOutGeneralSpecification\& specification, const TNetNetwork* network)

: TOutRetriever (specification, network)

fSpaceStorage(hosts, specification.GetRoot (), specification.GetName() + "." kSpacesuffix),

fTimeStorage(hosts, specification.GetRoot (), specification. GetName() + "." + kTimesuffix)

1 
// Perform the retrieval on the specified writers. void TOutSummaryRetriever::Retrieve(TOutWriter\& spaceWriter, TOutWriter\& timeWriter, bool sort)

\{

BasicRetrieve (fSpacestorage, spaceWriter, sort);

BasicRetrieve (fTimestorage, timewriter, sort):

\section{Y. TOutTextWriter Class}

\section{TextWriter.h}

$1 /$ Project: TRANSIMS

// Subsystem: Simulation Output

// \$RCSfile: TextWriter.h,v \$

// \$Revision: $0.5 \$$

$1 /$ \$Date: 1996/06/19 17:42:54 \$

$1 /$ \$State: Stab \$

// \$Author: bwb \$

// U.S. Government Copyright 1995

$/ /$ All rights reserved

\#ifndef TRANSIMS OUT TEXTWRITER

\#define TRANSIMS_OUT_TEXTWRITER

$/ /$ Include standard $\mathrm{C}++$ header files.

\#include 〈fstream.h>

1/ Include Booch Components header files.

\#include <BCStoreM.h>

\#include 〈BCCollu.h〉

$1 /$ Include TRANSIMS header files.

\#include <GBL/Globals.h>

\#include <OUT/Writer.h>

// A text writer puts simulation output data into a formatted text file. The $1 /$ exception ToutWriterFailure is thrown if an operation fails.

class ToutTextWriter [

: public ToutWriter

public:

// Open the specified file for writing, including the record header ToutTextwriter/const string file, const string\& delimiter = "\t", bool inclideHeader $=$ FALSE ;

$1 /$ Write the specified record with fields in the given order. void Write(const TOutRecorda record);

void Write(const ToutRecord\& record, const Fieldcollection\& fields);

private:

$1 /$ Each text writer is connected to a file.

ofstream fout:

// Each text writer has a delimjter.

string fDelimiter:

// A text writer may have to include header information.

1:

bool fIncludeHeader:

\# Endif // TRANSIMS_OUT_TEXTWRITER 


\section{TextWriter.C}

$/ /$ Project: TRANSIMS

// Subsystem: Simulation Output

// \$RCSfile: TextWriter.C, $v \$$

$/ /$ SRevision: $0.6 \$$

// \$Date: 1996/06/19 17:42:43\$

// SState: Stab $\$$

// \$Author: bwb \$

// U.S. Government Copyright 1995

// All rights reserved

// Include TRANSIMS header files. \#include <OUT/TextWriter.h>

// Open the specified file for writing, including the record header TOutTextWriter: : TOutTextWriter(const string file, const string\& delimiter, bool includeHeader)

: fout (file),

fDelimiter(delimiter)

fIncludeHeader (includeHeader)

1

if (! fout.rdbuf()->is_open())

) throw TOutWriterFailure("Cannot open file.");

// Write the specified record.

void TOutTextWriter: :Write(const TOutRecord\& record)

i

FieldCollection fields;

for (TOutRecord:: FieldMapIterator $i=$ record.GetIterator(); !i.IsDone(); i. Next ())

fields. Append (*i.CurrentItem());

\}

Write(record, fields);

void TOutTextWriter: Writelconst TOutRecord\& record, const FieidCollection\& fields)

const TOutRecord: : FieldMap\& map = record.GetMap ( ):

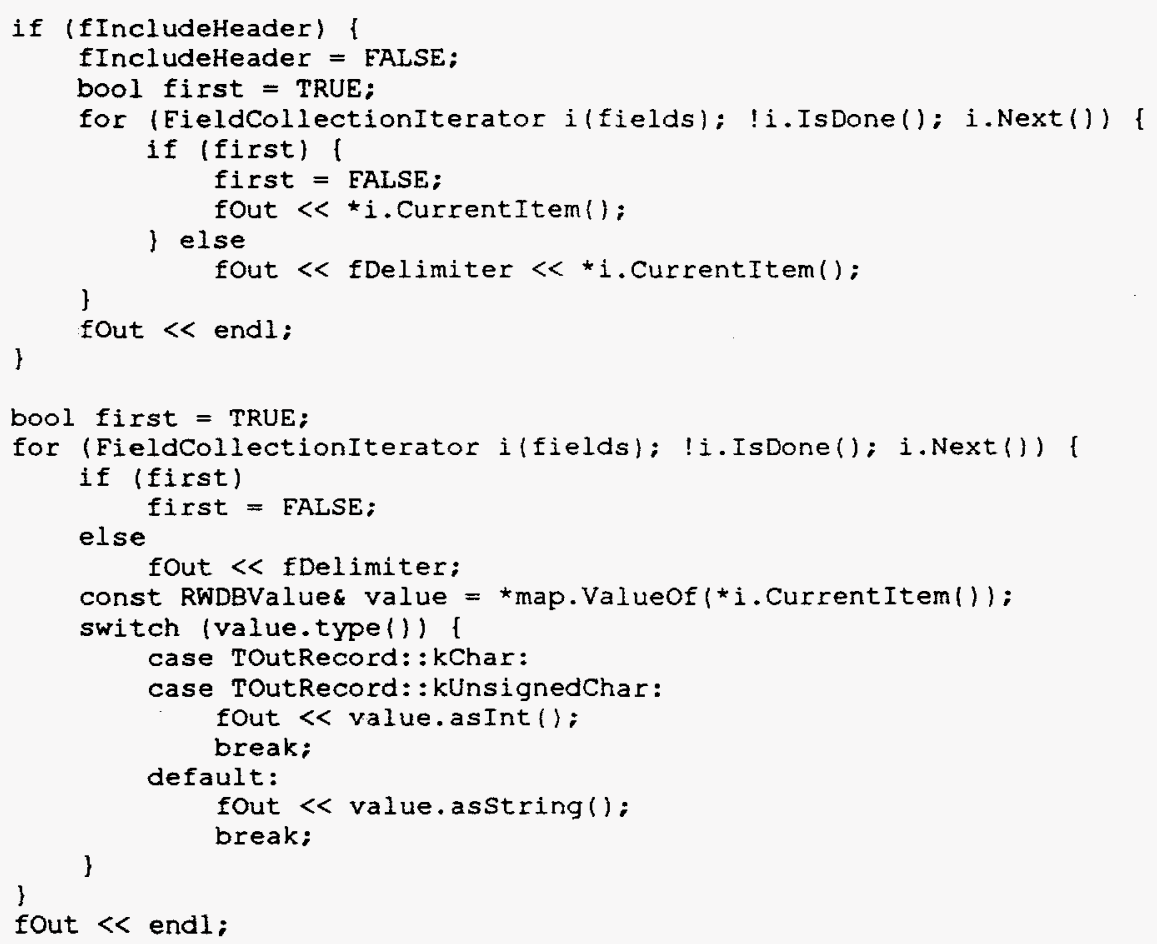




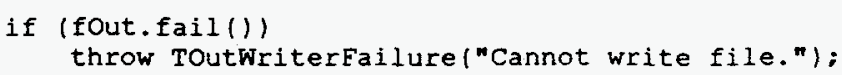

\section{Z. TOutVehicleObserver Class}

\section{VehicleObserver.h}

$1 /$ Project: TRANSIMS

1/ Subsystem: Simulation Output

// SRCsfile: Vehicleobserver.h, $v \$$

// \$Revision: $0.4 \$$

// \$Date: 1996/06/19 17:43:24 \$

$1 /$ \$State: Stab \$

// SAuthor: bwb \$

// U.S. Government Copyright 1995

// All right reserved

\#ifndef TRANSIMS_OUT_VEHOBS

\#define TRANSIMS OUT VEHOBS

// Include TRANSIMS header files.

\#include "OUT/Observer.h"

\#include "NET/Id.h"

// A vehicle observer observes data related to vehicles.

class TOutVehicleobserver : public TOutObserver

public:

// Construct a vehicle observer.

TOutVehicleobserver (OutObserverId id);

// Destruct a vehicle observer. virtual -TOutVehicleobserver():

protected:

// Define values for the fields being observed.

// The Observe method will use these methods.

1/ Define the vehicle's id.

void SetId(UINT id):

// Define the id of the link the vehicle is on. void SetLink(NetLinkId link);

// Define the lane number the vehicle is on. void SetIane (NetLaneNumber lane):

// Define the vehicie's distance from a node. void SetDistance (REA.L distance):

// Define the id of the node the distance is measured from. void SetNode(NetNodeId node):

// Define the vehicle's velocity. void SetVelocity(REAI velocity);

// Define the vehicle's status. void SetStatus(BYTE status):

;

\#endif // TRANSIMS_OUT_VEHOBS 


\section{VehicleObserver.C}

$1 /$ Project: TRANSIMS

// Subsystem: simulation output

// \$RCsfile: Vehicleobserver.c, v \$

// SRevision: 0.5 \$

// \$Date: 1996/06/19 17:43:08 \$

// \$State: Stab \$

// \$Author: bwb \$

// U.S. Government Copyright 1995

// All right reserved

1/ Include TRANSIMS header files.

"include "OUT/Vehicleobserver.h"

"include "OuT/Record.h"

\#include "OUT/Names.h"

// Construct a vehicle observer.

ToutVehicleobserver: : TOutVehicleobserver (OutObserverId id)

: Toutobserver(id)

SetTime(-99.):

SetId $(-99)$;

SetLink $(-99)$

SetLane $(-99)$ :

SetNode $(-99)$

SetDistance $(-99.0)$ :

SetVelocity $(-99.0)$ :

Setstatus (25E):

)

$1 /$ Destruct a vehicle observer.

TOutVehicleobserver: : TOutVehicleobserver()

l

\}

$1 /$ Define the vehicle's id.

void TOutVehicleobserver: SetId(UINT id)

1

GetRecord(). SetField(kVehicleField, id);

// Define the id of the link the vehicle is on.

void TOutVehicleobserver: :SetLink (NetLinkId id)

\{

GetRecord(). SetField(kLinkField, id);

// Define the lane number the vehicle is on.

void TOutVehicleObserver: :SetLane (NetLaneNumber n)

l

GetRecord().SetField(kLaneField, $n$ );

// Define the vehicle's distance from a node.

void TOutVehicleobserver: :SetDistance(REAL d)

f

GetRecord().SetField(kDistanceField, d):

\}

$1 /$ Define the id of the node the distance is measured from.

void TOutVehicleobserver: : SetNode (NetNodeId id)

l

GetRecord().SetField(kNodeField, id); 


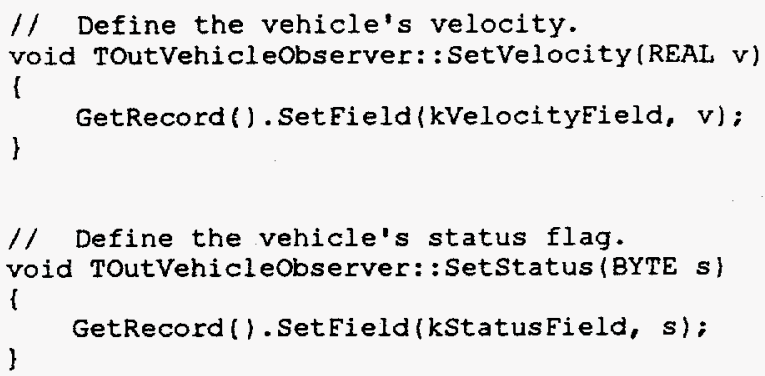

\section{AA. TOutWriter Class}

\section{Writer.h}

$1 /$ Project: TRANSIMS

// Subsystem: Simulation Output

// \$RCSfile: Writer.h,v \$

// SRevision: 0.4 \$

// \$Date: 1996/06/19 17:43:56 \$

$1 /$ SState: Stab \$

// SAuthor: bwb \$

$/ /$ U.S. Government Copyright 1995

// All rights reserved

\#ifndef TRANSIMS OUT WRITER

\#define TRANSIMS_OUT_WRITER

$/ /$ Include TRANSIMS header files.

\#include $\langle$ GBL/Globals.h>

\#include <OUT/Exception.h>

\#include 〈OUT/Record.h〉

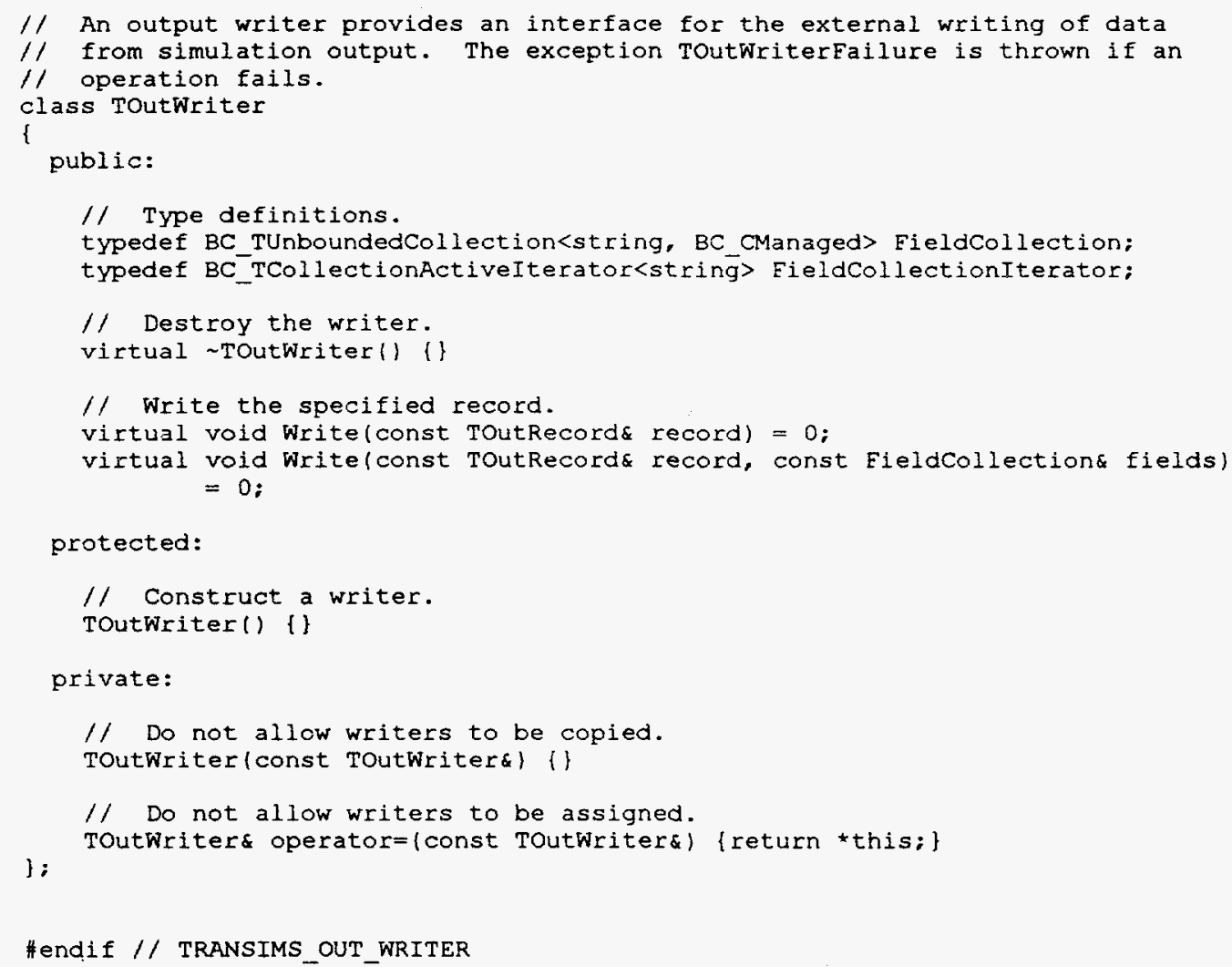




\section{BB. Type Definitions}

\section{Id.h}

// Project: TRANSIMS

// Subsystem: Simulation Output

// SRCSfile: Id.h, $\$$

// SRevision: $0.4 \$$

// SDate: 1996/06/19 17:26:19\$

// \$state: Stab \$

// \$Author: bwb \$

$/$ U.S. Government Copyright 1995

// All right reserved

\#ifndef TRANSIMS OUT ID

\#define TRANSIMS_OUT_ID

// Include TRANSIMS header files. \#include "GBL/Globals.h"

// A processor id is four bytes long. typedef DWORD OutProcessorId;

// An observer id is four bytes long. tyoedef DWORD outobserverId:

"endif // TRANSIMS_OUT_ID

\section{Names.h}

// Project: TRANSIMS

// Subsystem: Simulation Output

// SRCSfile: Names.h, $\mathrm{v}$ \$

// \$Revision: $0.5 \$$

// \$Date: 1996/06/19 17:31:05 \$

// \$State: Stab \$

$1 /$ SAuthor: bwb $\$$

$1 /$ U.S. Government Copyright 1995

$/ /$ All rights reserved

\#ifndef TRANSIMS OUT NAMES \#define TRANSIMS_OUT_NAMES

// The suffixes.

static const string kVehicleSuffix = "veh";

static const string kIntersectionsuffix = "int";

static const string ksignalsuffix = "sig";

static const string kLinkSuffix = "lnk";

static const string kspaceSuffix $=$ "spa";

static const string kTimeSuffix $=$ "tim";

// Field names.

static const string kTimeEield = "TIME";

static const string kLinkField = "LINK";

static const string kVehiclefield = "VEHICLE";

static const string kLaneEield = "LANE":

static const string kDistanceField = "DISTANCE";

static const string kNodeField = "NODE";

static const string kVelacityField = "VELOCITY";

static const string kStatusField = "STATUS";

static const string kQIndexField = "QINDEX";

static const string kSignalfield = "SIGNAL";

static const string kCountField = "COUNT";

static const string kSumField $=$ "SUM";

static const string kSumSquaresField = "SUMSQUARES"; 


\section{APPENDIX: Test Program}

This appendix contains the complete $\mathrm{C}++$ source code and the database import file for the simulation output subsystem test program.

\section{A. Test.C}

$1 /$ Project: TRANSIMS

// Subsystem: Simulation Output

// \$RCSfile: Test.C, $\mathrm{v}$ \$

// \$Revision: 0.7 \$

// \$Date: 1996/06/19 17:41:35\$

$1 /$ \$state: Stab \$

// \$Author: bwb \$

// U.S. Government Copyright 1995

// All rights reserved

// Include Standard C++ header files.

\#include <iostream.h>

// Include TRANSIMS header files.

\#include <GBL/Globals.h>

\#include <DBS/Exception.h>

\#include <OUT/Exception.h>

$/ /$ Function prototypes.

extern bool TestRecord():

extern bool Teststorage ();

extern bool TestTextwriter();

extern bool TestGeneralspecification();

extern bool TestEvolutionRetriever ();

// Main program.

int main(int, char*[])

l

try

bool fail = FALSE;

cout $<$ "Simulation Output Subsystem Tests"

$<$ "[\$Revision: 0.7 \$]"

$<$ endl;

fail $\mid=$ ! TestRecord () ;

fail $=$ ! TestStorage (\} ;

fail l= !TestTextwriter():

fail l= ! TestGeneralspecification():

fail I= ! TestEvolutionRetriever();

cout « $\left(\right.$ fail ? " $E^{n}$ : " No $\left.f^{n}\right) \ll$ "ailures occured." « endl;

\} catch(const ToutException\& exception) (

cout $<<$ endl;

cout $<$ "Unexpected simulation output exception: " $<$

exception. GetMessage () $<$ endl;

\} catch(const TDbExceptions exception) (

cout $<$ endl;

cout $\ll$ "Unexpected database exception: " $<$ exception.GetMessage() $<<$ endi:

$|\operatorname{catch}(\ldots)|$ 


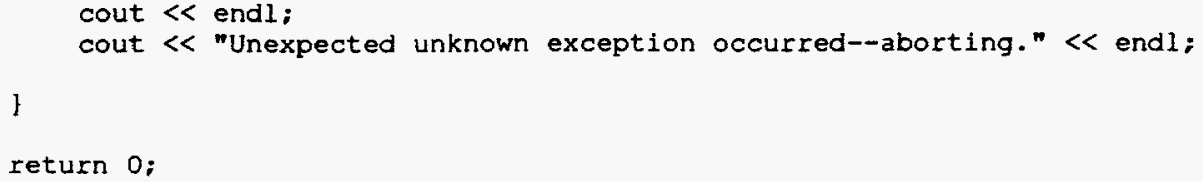

B. TestEvolutionRetriever.C

// Project: TRANSIMS

// Subsystem: Simulation Output

// SRCSfile: TestEvolutionRetriever.C,v $\$$

// \$Revision: 0.5 \$

/f \$Date: 1996/06/19 17:41:48 \$

// \$state: Stab \$

1/ SAuthor: bwb \$

// U.S. Government Copyright 1995

// All rights reserved

// Include Standard C++ header files.

\#include 〈fstream.h〉

\#include <iastream.h>

// Include TRANSIMS header files.

\#include <DBS/Exception.h>

" include <DBS/Directory.h>

\#include <DBS/Source.h>

\#include <DBS/Table h>

\#include <OUT/SpecificationReader.h>

\#include <OUT/GeneralspecificationReader.h>

\#include <OUT/Generalspecification.h>

\#include <OUT/EvolutionRetriever.h>

\#include <OUT/TextWriter.h>

// Test evolution retriever class.

bool TestevolutionRetriever()

cout $\ll "$ Evolution Retriever Class Tests"
$\ll<$ [\$Revision: $0.5 \$]^{n}$

$\ll$ endl:

bool anyFail = FALSE;

bool fail

TOutStorage: :HostSet hosts (HashValue);

hosts. Add ("host1"):

hosts. Add ("host2"):

TOutStorage storage/hosts, "/home/projects/transims/test/NET",

"sample.veh", ToutStorage: :kwritel.

TOutRecord record;

record.SetField("TIME", 10);

record.SetField ("LINK", 11);

record. SetField("VELOCITY", 5);

storage.WriteHeader ("host1", record);

storage.Write("host1", record);

record.SetEield("TIME", 60);

record.SetField("LINK", 11);

record.SetField ("VELOCITY", 6);

storage.Write("host1", record);

record.Setfield("TIME", 61);

record.SetField ("LINK", 12);

record. SetField("VELOCITY", 7)

storage.Write("host1", record);

record.SetField ("TIME", 65):

record.SetEield("IINK", 10): 
record.SetField ("VELOCITY", B); storage.Write("host1", record);

record.SetField ("TIME", 9);

record.SetField("LINK", 12);

record. SetField("VELOCITY", 4 ):

storage.WriteHeader ("host2", record);

storage.Write("host2", record);

record.SetField("TIME", 60):

record.SetField ("LINK", 12);

record.SetField ("VELOCITY", 3):

storage.Write("host $2 "$ ", record);

record.SetField("TIME", 65);

record. SetField ("LINK", 12);

record.SetField ("VELOCITY", 2);

storage.Write("host2", record);

record.SetField("TIME", 65);

record.SetField("LINK", 11);

record.SetField ("VELOCITY", 1);

storage.Write("host2", record);

record.SetField("TIME", 65):

record.SetField("LINK", 10);

record.SetField("VELOCITY", 0);

storage.Write("host2", record);

record. SetField "TIME", 65):

record.SetField "LINK", 11);

record.SetField("VELOCITY", 0):

1

storage.Write("host2", record):

TDbDirectory directory(TDbDirectoryDescription("IOC-1")):

TDbSource generalsourceldirectory,

directory.GetSource ("Output Specification")/:

TDbSource nodeSourceldirectory,

directory.GetSource ("Output Node Specification")!:

TDbsource linksource (di rectory,

directory.GetSource ("Output Link Specification"));

TDbTable generalTablelgeneralsource, generalsource.GetTable("Sample Output " "Specification Table"));

TDbTable nodeTable (nodeSource, nodeSource.GetTable("Sample Output Node " "Specification Table")! :

TDbTable linkTable(linkSource, linkSource.GetTable("Sample Output Link" "Specification Table")/;

TOutGeneralSpecificationReader reader(TOutSpecificationReader/generalTable, nodeTable, linkTable));

reader.Reset ():

TOutStorage: : HostSet hosts (HashValue);

hosts. Add ("host1");

hosts. Add ("host2"):

TOutGeneralspecification specification(reader):

TOutEvolutionRetriever retriever(hosts, specification);

ISSUE (KPB) Intersection and signal information is not really being tested. Sample intersection and signal storages are duplicates of the vehicle storage; therefore the sample.int.txt and sample.sig.txt files produced here are (correctly) empty.

TOutTextWriter* vehicleWriter $=$ new TOutTextWriter("/home/projects/transims/test/NET/sample.veh.txt", ", ", TRUE):

TOutTextWriter intersectionwriter $=$ new

TOutTextWriter/"/home/projects/transims/test/NET/sample.int.txt", ", ", TRUE);

TOutTextwriter* signalWriter $=$ new TOutTextWriter ("/home/projects/transims/test/NET/sample.sig.txt". ", ", TRUE):

retriever.Retrieve(*vehicleWriter, *intersectionWriter, *signalWriter); delete vehiclewriter; 


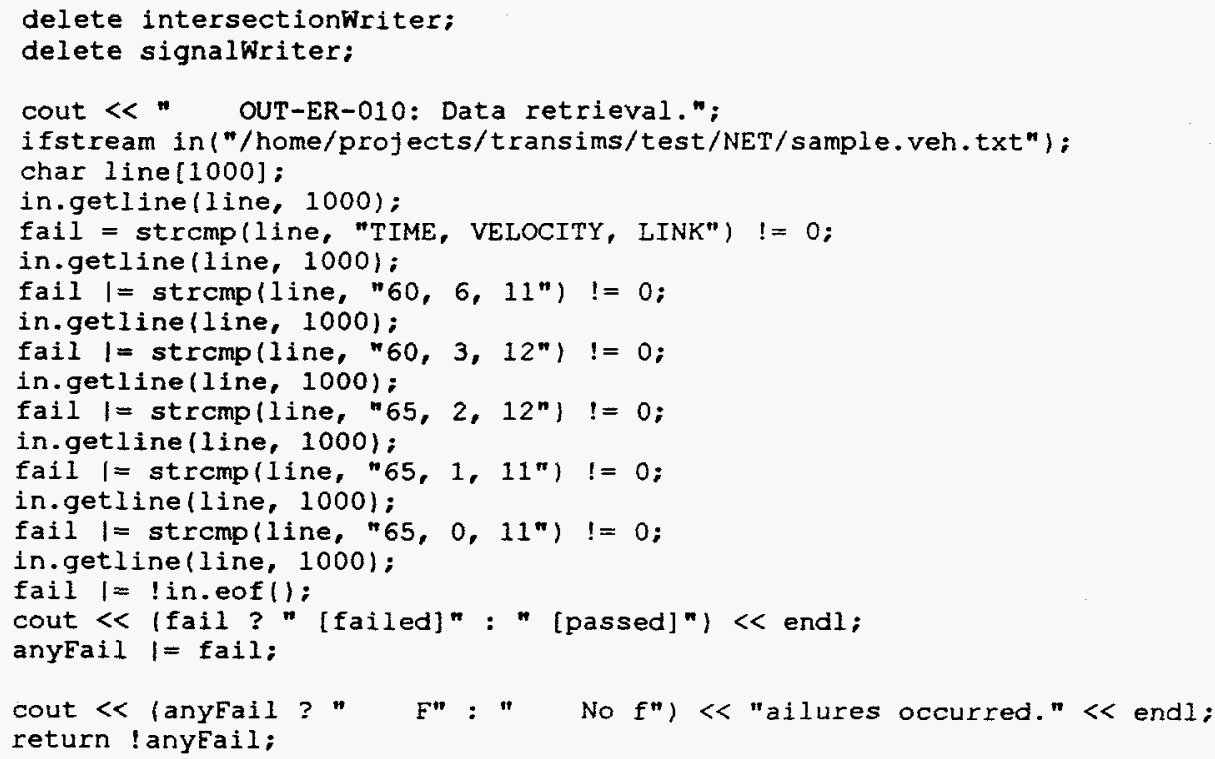

\section{TestGeneralSpecification.C}

$1 /$ Project: TRANSIMS

$1 /$ Subsystem: Simulation Output

// SRCSfile: TestGeneralspecification.C,v \$

$1 /$ SRevision: 0.5 \$

// SDate: 1996/06/19 17:42:00\$

// SState: Stab \$

// \$Author: bwb $\$$

// U.S. Government Copyright 1995

// All rights reserved

// Include standard C++ header filles.

\#include <iostream.h>

// Include standard $C$ header files.

\#include <math.h>

// Include TRANSIMS header files.

\#include <DBS/Exception.h>

\#include <DBS/Di rectory. h>

\#include <DBS/Source. $h$ >

\#include <DBS/Table.h>

\#include <OUT/SpecificationReader.h>

\#include <OUT/GeneralspecificationReader.h>

\#include <OUT/Generalspecification.h>

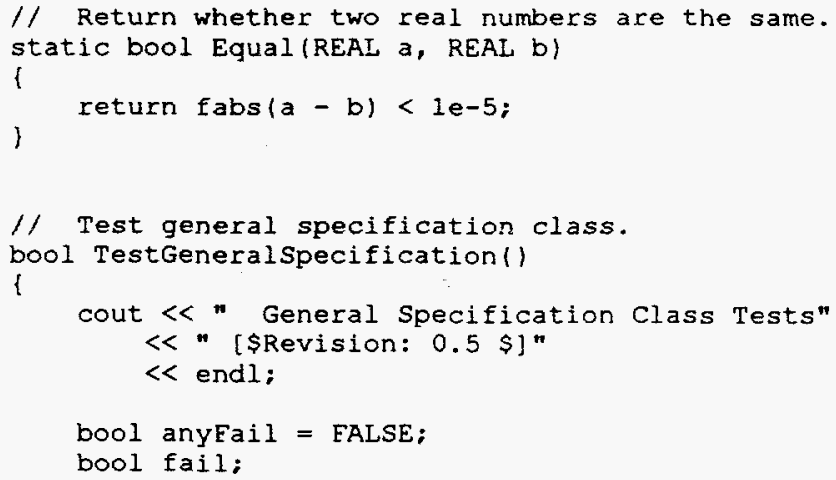


TDbDirectory directory(TDbDirectoryDescription("IOC-1"));

TDbsource generalsource/directory,

directory.GetSource ("Output Specification"));

TDbsource nodesourceldirectory,

directory.GetSource ("Output Node Specification")!;

TDbsource linksource (directory,

directory.GetSource ("Output Link Specification"));

TDbTable generalTablelgeneralsource, generalSource.GetTable("Sample Output " "Specification Table")l;

TDbTable nodeTable/nodeSource, nodeSource.GetTable/"Sample Output Node " "Specification Table") l;

TDbTable linkTablellinkSource, linkSource.GetTable!"Sample output Iink " "Specification Table")l;

TOutGeneralspecificationReader reader(TOutSpecificationReader(generalTable, nodeTable, linkTable)l:

reader.Reset ();

TOutGeneralspecification specification(reader);

cout $\ll "$ OUT-GS-010: Root retrieval.";

fail = specification. GetRoot ()$!=$ "/home/projects/transims/test/NET";

cout $<$ (fail ? " [failed]" : " [passed]") << endl;

anyFail I= fail;

cout $\ll$ oUT-GS-020: Name retrieval.";

fail = specification.GetName () != "sample";

cout $<$ (fail ? "[failed]" : " [passed]") $<$ endl;

anyFail $I=$ fail:

cout $\ll "$ OUT-GS-030: Time collection.";

fail = specification. CollectForTime (59) || !specification.CollectForTime $(60)$ 1) !specification.CollectEorTime(100) 11

specification. Collect ForTime(101) ||

!specification. Collect ForTime (120) 11

specification.CollectEorTime (121):

cout 《 (fail ? " [failed]" : " [passed]") 《 endl;

anyFail l= fail;

cout $\ll n$ OUT-GS-040: Time sampling." ;

fail = specification. SampleForTime $(50) \|$ !specification. SampleForTime(60)

I! !specification. SampleForTime(100) II

specification. SampleForTime(101) ||

!specification. SampleEorTime(120) ||

specification. SampleEorTime(121):

cout $<$ (fail? " [failed]" : "[passed]") $<$ endl;

anyEail |= fail;

cout $\ll "$ OUT-GS-050: Box length." ;

fail = !Equal (specification.GetBoxLength(), 150);

cout $\ll$ (fail? "[failed]" : "[passed]")" $<$ end];

anyFail |= fail;

cout $\ll "$ OUT-GS-060: Point collection.";

fail = specification. CollectForPoint (TGeoPoint $(0,0))||$

ispecification. CollectEorPoint (TGeoPoint $(1,2)$ ) $\mid 1$

!specification. CollectForPoint (TGeoPoint $(500,600)) 11$

!specification. CollectForPoint (TGeoPoint (1000, 2000)) |1

specification. CollectForPoint (TGeoPoint(1001, 2001));

cout $<$ (fai] ? "[failed]" : " [passed]") $<$ endl;

anyFail l= fail;

cout $\ll "$ OUT-GS-070: Node collection.";

fail = specification. CollectForNode (11) il

ispecification. Collect ForNode(1) ||

!specification. Collect ForNode (2);

cout < (fail? "[failed]" : "[passed]") < endl;

anyFail l= fail;

cout $\ll "$ OUT-GS-080: Link collection.";

fail = specification.CollectForLink(1) $\mid$

!specification.CollectForLink(11) । 


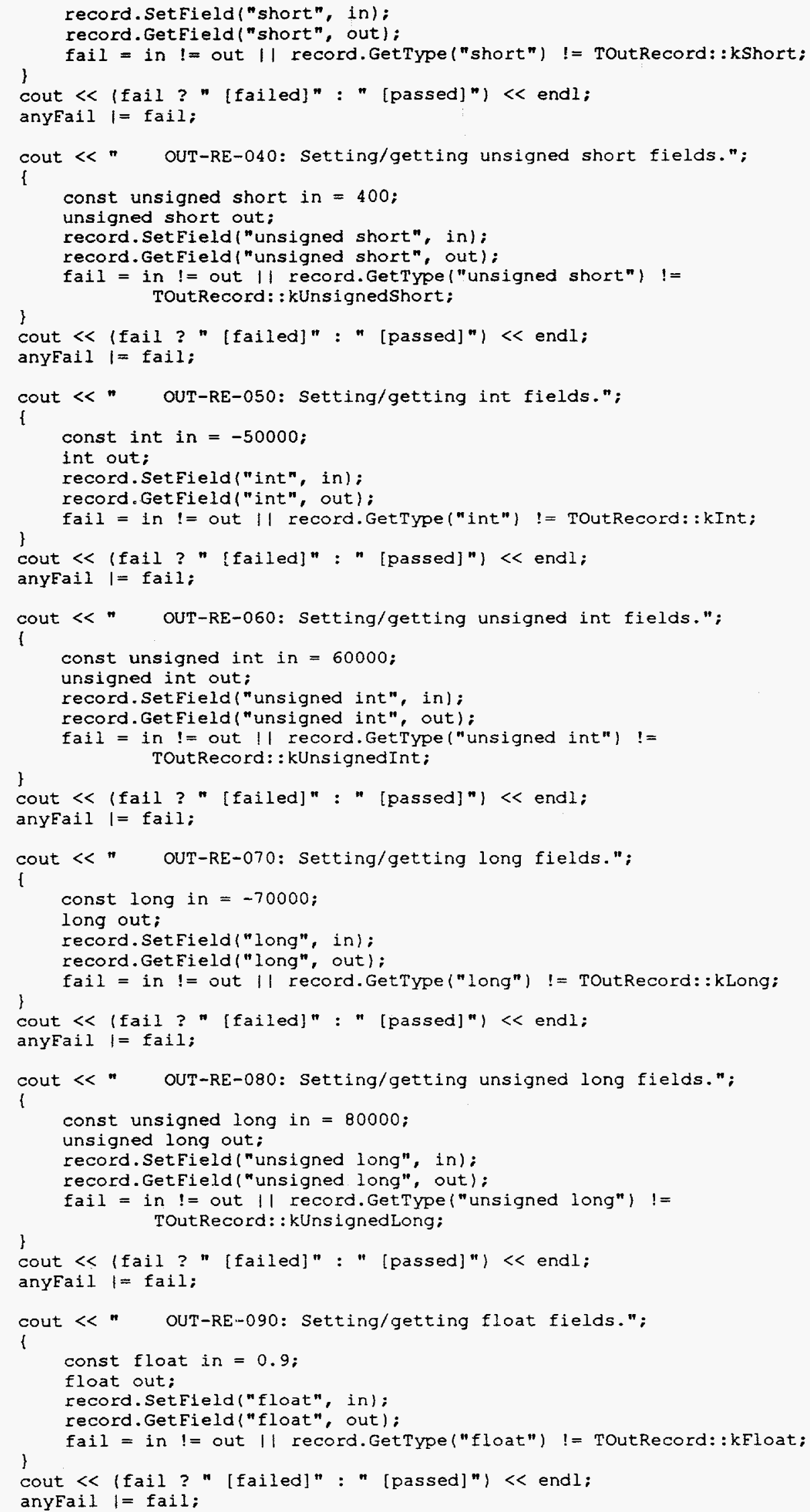




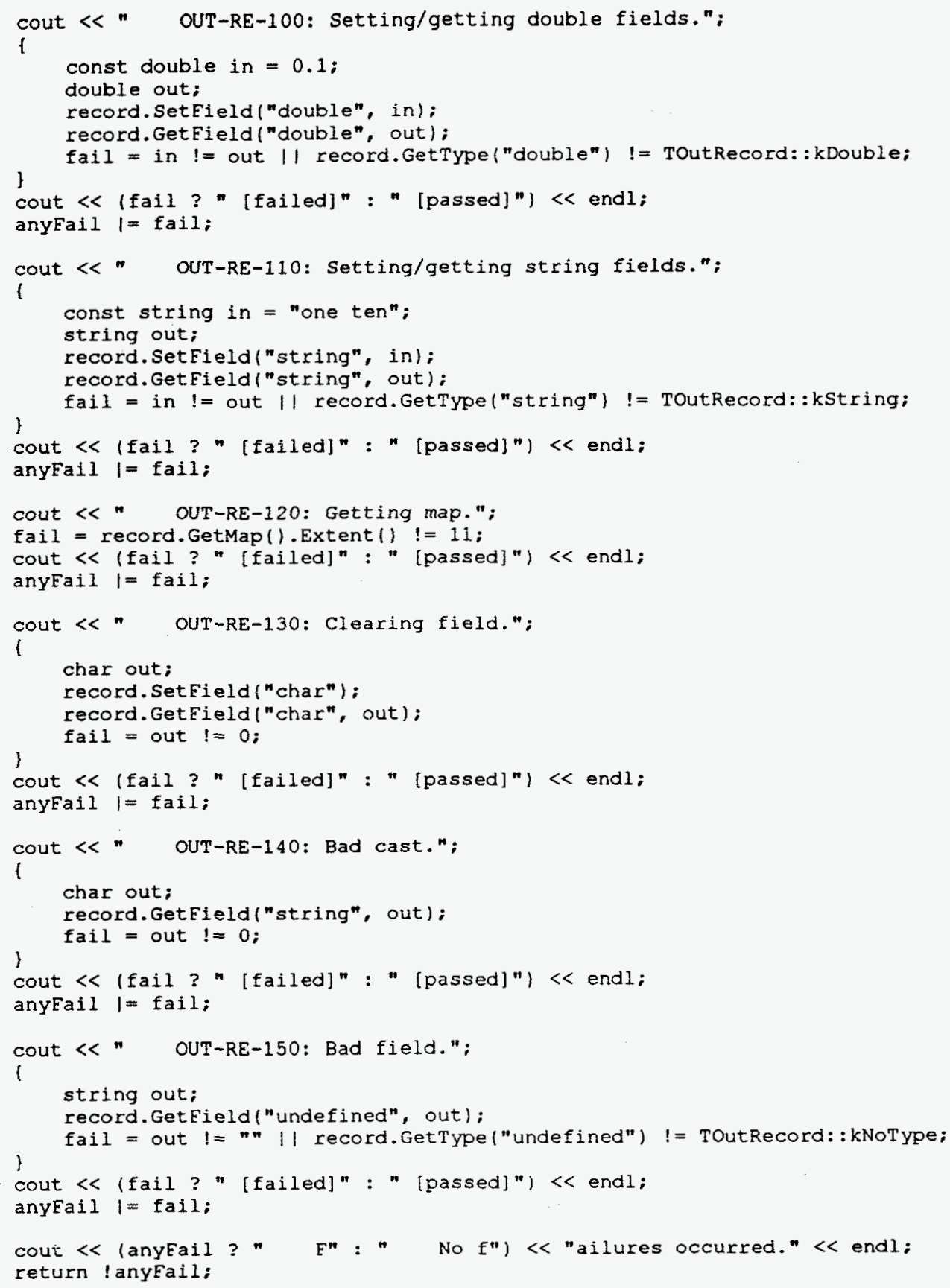

\section{E. TestStorage.C}

$1 /$ Project: TRANSIMS

$/ /$ Subsystem: Simulation Output

// \$RCSfile: Teststorage.C,v \$

// SRevision: 0.5 \$

// SDate: 1996/06/19 17:42:21 \$

// \$state: Stab \$

$1 /$ SAuthor: bwb \$

// U.S. Government Copyright 1995

// All rights reserved

// Include Standard C+t header files. 
\#include <iostream.h>

// Include TRANSIMS header files.

\#include <OUT/Storage.h>

$1 /$ Test storage class.

bool Teststorage()

[

cout $\ll "$ Storage Class Tests"

$<$ " [\$Revision: 0.5 \$]"

$<<$ endl;

bool anyEail = EALSE;

bool fail:

Toutstorage* stg;

TOutRecord* inrecl;

TOutRecord* inrec 2 ;

TOutRecord* outrec:

stg = new TOutstorage("/home/projects/transims/test/NET", "temp", TOutstorage: :kwrite);

cout $<$ " OUT-ST-010: Root retrieval.";

fail = stg->GetRoot () ! = "/home/projects/transims/test/NET" ;

cout $<$ (fail? " [failed]" : "[passed]") $<$ endl;

anyFail i= Fail;

cout $<"$ OUT-ST-020: Name retrieval." ;

fail = stg->GetName () != "temp" :

cout $<$ (fail? " [failed]" : "[passed]") < endl:

anyEail I=fail:

cout < " OUT-ST-030: Host retrieval." ;

TOutStorage: :HostSet hosts $=$ stg->GetHosts ();

(

TOutStorage: :HostSetIterator ilstg->GetHosts());

fail $=\star \dot{i}$. CurrentItem() $!=$ "local";

i. Next ():

\}

fail $l=$ ! i. IsDone();

cout $<$ (fail ? "[failed]" : " [passed]") $<$ endl:

anyEatl = fail;

inrecl = new TOutRecord (1)

inrecl->SetField "One", 1.0);

inrec1->SetField ("Two", 2):

inrec1->SetField ("Three", "three");

inrec2 = new TOutRecord 1 );

inrec2->SetField ("One", "one"):

inrec2->SetField ("Two", 2.0);

inrec2->SetField ("Three", 3);

stg->WriteHeader $($ *inrec1):

stg->Write (*inrecl):

stg $\rightarrow$ Write $(*$ inrec 2$\}$;

const long pos = stg->Getoffset ():

delete stg;

hosts.Clear();

hosts.Add ("local");

stg $=$ new TOutStorage(hosts, "/home/projects/transims/test/NET", "temp");

outrec = new TOutRecord():

cout $\ll n$ OUT-ST-040: Check for not end-of-file." ;

fail = stg->AtEnd ( ) :

cout $<$ (fail ? "[failed]" : "[passed]") $<$ endl;

anyEail l= fail:

cout $<"$ OUT-ST-050: Header write/read." ; 


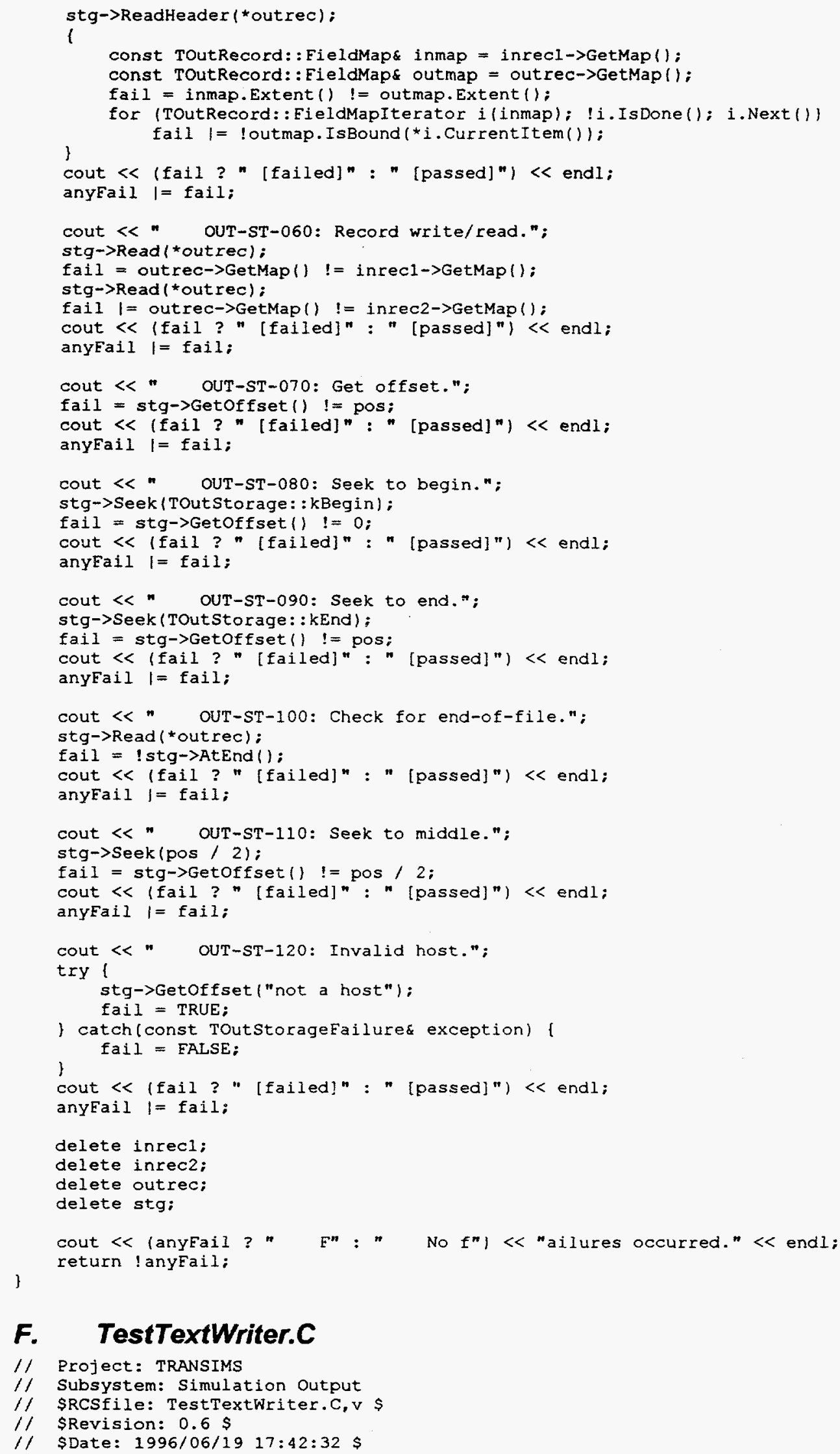

\section{F. TestTextWriter.C}

// Project: TRANSIMS

f) Subsystem: Simulation output

// SRCSfile: TestTextWriter.C, $\mathrm{v} \$$

// SDate: 1996/06/19 17:42:32\$

// SRevision: $0.6 \$$ 


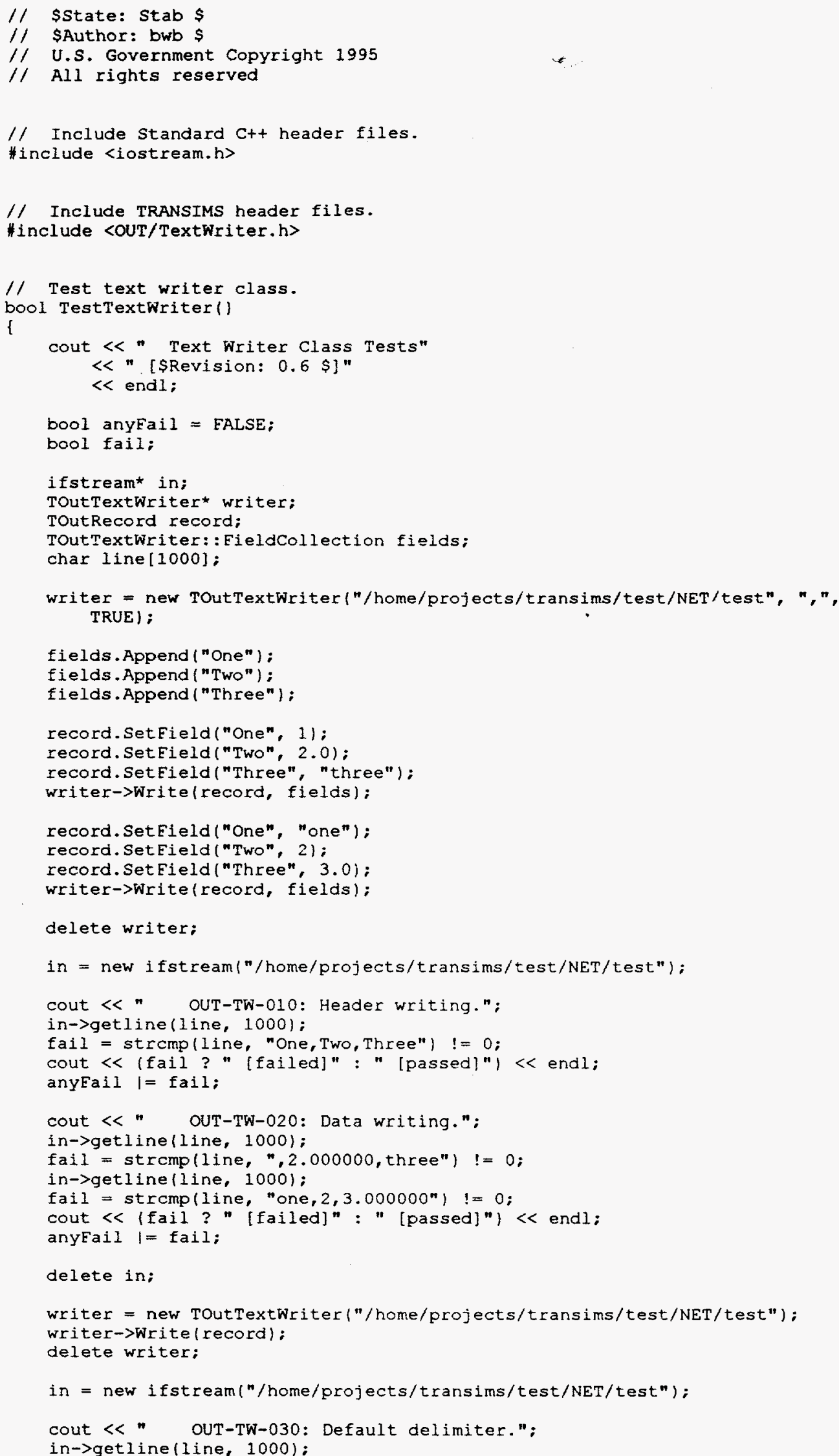

